\title{
Synthesis of 2(5H)-Furanone Derivatives with Symmetrical and Unsymmetrical Bis-1,2,3-triazole Structure
}

Yuehe Tan, Jianxiao Li, Jingpei Huo, Fuling Xue, Zhaoyang Wang*

School of Chemistry and Environment, South China Normal University; Key Laboratory of Theoretical Chemistry of Environment, Ministry of

Education, Guangzhou, 510006, P. R. China

*Corresponding Author E-mail: wangzy@scnu.edu.cn or wangwangzhaoyang@tom.com; fax: 8620-39310187.

\section{SUPPORTING INFORMATION}

\section{Full experimental detail}

(Characterization spectra of 32 new compounds $2 \mathbf{a}-\mathbf{2 h}, \mathbf{3 a - 3 h}, \mathbf{5 a a - 5 b h}$. For each compound, the former is ${ }^{1} \mathrm{H}$ NMR, and the latter is ${ }^{13} \mathrm{C}$ NMR.) 


\section{EXPERIMENTAL}

\section{General}

Melting points were determined on an X-5 digital melting points apparatus and were uncorrected. Infrared spectra were recorded on a Bruker Vector 33 FT-IR instrument by liquid film method in the absorption range of 4000-400 $\mathrm{cm}^{-1} .{ }^{1} \mathrm{H}$ and ${ }^{13} \mathrm{C}$ NMR spectra were obtained in $\mathrm{CDCl}_{3}$ on a Varian $\mathrm{DRX}-400 \mathrm{MHz}$ spectrometer and tetramethylsilane (TMS) was used as an internal standard. UV absorption peaks were measured by Shimazu UV-2550 ultraviolet absorption detector with dichloromethane as a solvent. Optical rotations were determined with an Autopol IV polarimeter in $\mathrm{CH}_{2} \mathrm{Cl}_{2}$ in a $10 \mathrm{~cm}$ cell. Elemental analysis was performed on a Thermo Flashea TM 112 elemental analyzer. The mass spectra (MS) were recorded on Thermo LCQ DECA XP MAX mass spectrometer.

All reagents and solvents were commercially avail- able and used as received. The intermediates $N$-[(5S)-5-alkoxy-2(5H)-furanonyl] amino acid propargyl esters $\mathbf{1}$ were prepared according to the literature. ${ }^{[31]}$

\section{General procedure for the synthesis of target compounds 2}

A flame-dried flask was charged with $1 \mathrm{mmol} N$-[(5S)-5-alkoxy-2(5H)-furanonyl] amino acid propargyl esters $\mathbf{1}$, $0.5 \mathrm{mmol} 1,4$-diazidobutane, ${ }^{[42]} 0.05$ mmol $\mathrm{Cu}(\mathrm{OAc})_{2} \bullet \mathrm{H}_{2} \mathrm{O}$ and $0.1 \mathrm{mmol} \mathrm{Cu}$ in $\mathrm{CH}_{3} \mathrm{CN}(5 \mathrm{~mL})$. The mixture was stirred at room temperature under $\mathrm{N}_{2}$ atmosphere and monitored by TLC. Once the reaction was completed (about $16 \mathrm{~h})$, the reaction mixture was quenched with a saturated aqueous solution of $\mathrm{NH}_{4} \mathrm{Cl}(20 \mathrm{~mL})$ and extracted with ethyl acetate $(3 \times 30 \mathrm{~mL})$. The organic layer was dried over $\mathrm{MgSO}_{4}$, and concentrated in vacuo. The residue was purified by column chromatography using the mixture of petroleum ether and ethyl acetate as eluent to yield the desired compounds $\mathbf{2 a - 2 h}$ in $41-56 \%$ isolated yields.

(S,R,R,S,2S,2'S)-(1,1'-(butane-1,4-diyl)bis(1H-1,2,3-triazole-4,1-diyl))bis(methylene) bis(2-((S)-4-bromo-2-((1R,2S,5R)-2-isopropyl-5-methylcyclohexyloxy)-5-oxo2,5-dihydrofuran-3-ylamino)-3-methylbutanoate) (2a). Yellowish oil, yield $254 \mathrm{mg}(47 \%)$; $[\alpha]_{\mathrm{D}}{ }^{20}+112.5\left(c 0.027, \mathrm{CH}_{2} \mathrm{Cl}_{2}\right) ; \mathrm{UV}^{-v i s}\left(\mathrm{CH}_{2} \mathrm{Cl}_{2}\right) \lambda_{\max }: 270 \mathrm{~nm}$; ${ }^{1} \mathrm{H} \mathrm{NMR}(400$ $\left.\mathrm{MHz}, \mathrm{CDCl}_{3}-\mathrm{TMS}\right) \delta$, ppm: $0.85(\mathrm{~d}, J=8.0 \mathrm{~Hz}, 6 \mathrm{H}), 0.86-1.01(\mathrm{~m}, 26 \mathrm{H}), 1.02-1.16(\mathrm{~m}, 4 \mathrm{H}), 1.31-1.46(\mathrm{~m}, 4 \mathrm{H}), 1.62-1.73(\mathrm{~m}, 4 \mathrm{H}), 1.89-2.01(\mathrm{~m}, 4 \mathrm{H}), 2.11-2.33(\mathrm{~m}, 6 \mathrm{H})$, 3.54-3.65 (ddd, $\left.J_{1}=4.0 \mathrm{~Hz}, J_{2}=4.0 \mathrm{~Hz}, J_{3}=4.0 \mathrm{~Hz}, 2 \mathrm{H}\right), 4.37-4.50(\mathrm{~m}, 4 \mathrm{H}), 4.80(\mathrm{~b}, 2 \mathrm{H}), 5.26-5.39(\mathrm{~m}, 6 \mathrm{H}), 5.76(\mathrm{~s}, 2 \mathrm{H}), 7.75(\mathrm{~s}, 2 \mathrm{H}) ;{ }^{13} \mathrm{C} \mathrm{NMR}\left(100 \mathrm{MHz}, \mathrm{CDCl} \mathrm{T}^{-\mathrm{TMS}}\right) \delta$, ppm: $15.7,17.3,18.0,20.9,22.1,22.7,25.8,27.1,31.5,31.9,33.8,42.2,47.9,49.4,58.5,59.8,77.4,83.2,99.9,124.3,141.8,157.3,167.9,170.5 ;$ IR $\left(\right.$ neat) $v, \mathrm{~cm}^{-1}: 3379$, 
3143, 3082, 2951, 2923, 2868, 1745, 1649, 1523, 1460, 1317, 1193, 1133, 952, 628; ESI-MS m/z (\%): 1081 ([M+H $\left.]^{+}, 44\right), 1103\left([\mathrm{M}+\mathrm{Na}]^{+}, 100\right)$; Anal. Calcd for $\mathrm{C}_{48} \mathrm{H}_{72} \mathrm{Br}_{2} \mathrm{~N}_{8} \mathrm{O}_{10}$ : C 53.33, H 6.71, N 10.37, Found: C 53.29, H 6.76, N 10.32.

(R,S,R,S,2S,2'S)-(1,1'-(butane-1,4-diyl)bis(1H-1,2,3-tri-azole-4,1-diyl))bis(methylene) bis(2-((S)-4-bromo-2-((1R,2S,5R)-2-isopropyl-5-methylcyclohexyloxy)-5oxo-2,5-dihydrofuran-3-ylamino)-3-phenylpropanoate) (2b). Yellowish oil, yield $306 \mathrm{mg}(52 \%) ;[\alpha]_{\mathrm{D}}{ }^{20}+51.0\left(c 0.096, \mathrm{CH}_{2} \mathrm{Cl}_{2}\right) ; \mathrm{UV}^{-v i s}\left(\mathrm{CH}_{2} \mathrm{Cl}_{2}\right) \lambda_{\text {max }}: 271 \mathrm{~nm}$; ${ }^{1} \mathrm{H} \mathrm{NMR}$ (400 MHz, CDCl 3 -TMS) $\delta$, ppm: $0.73(\mathrm{~d}, J=4.0 \mathrm{~Hz}, 6 \mathrm{H}), 0.76-0.93(\mathrm{~m}, 14 \mathrm{H}), 0.94-1.10(\mathrm{~m}, 4 \mathrm{H}), 1.27-1.43(\mathrm{~m}, 4 \mathrm{H}), 1.57-1.72(\mathrm{~m}, 4 \mathrm{H}), 1.83-2.19(\mathrm{~m}, 8 \mathrm{H}), 3.04-3.26(\mathrm{~m}, 4 \mathrm{H})$, 3.37-3.51 (m, 2H), 4.32-4.50 (m, 4H), 4.84-5.52 (m, 10H), 7.00-7.14 (m, 4H), 7.19-7.35 (m, 6H), $7.63(\mathrm{~s}, 2 \mathrm{H}) ;{ }^{13} \mathrm{C} \mathrm{NMR}\left(100 \mathrm{MHz}, \mathrm{CDCl} \mathrm{N}_{3} \mathrm{TMS}\right) \delta$, ppm: 15.6, 21.0, 22.1, 22.6, 25.5, 27.1, 31.5, 33.9, 39.8, 42.3, 47.8, 49.4, 55.8, 58.8, 77.3, 83.1, 99.8, 124.5, 127.7, 128.8, 129.4, 134.4, 141.7, 156.8, 167.6, 170.2; IR (neat) v, cm ${ }^{-1}$ 3368, 3137, 3066, 3027, 2945, 2923, 2863, 1742, 1646, 1520, 1495, 1451, 1322, 1182, 1117, 955, 744, 697, 648; ESI-MS m/z (\%): 1177 ([M+H] $\left.]^{+}, 30\right), 1199\left([\mathrm{M}+\mathrm{Na}]^{+}, 100\right)$; Anal. Calcd for $\mathrm{C}_{56} \mathrm{H}_{72} \mathrm{Br}_{2} \mathrm{~N}_{8} \mathrm{O}_{10}$ : C 57.14, H 6.17, N 9.52, Found: C 57.19, H 6.14, N 9.60.

$(R, R, S, S)-(1,1$ '-(butane-1,4-diyl)bis(1H-1,2,3-triazole-4,1-diyl))bis(methylene) bis(6-((S)-4-bromo-2-((1R,2S,5R)-2-isopropyl-5-methylcyclohexyloxy)-5-oxo-2,5-dihydrofuran-3-ylamino)hexanoate) (2c). Yellowish oil, yield $311 \mathrm{mg}(56 \%) ;[\alpha]_{\mathrm{D}}{ }^{20}+84.8\left(c 0.031, \mathrm{CH}_{2} \mathrm{Cl}_{2}\right) ; \mathrm{UV}^{-\mathrm{vis}}\left(\mathrm{CH}_{2} \mathrm{Cl}_{2}\right) \lambda_{\max }: 273 \mathrm{~nm} ;{ }^{1} \mathrm{H} \mathrm{NMR}\left(400 \mathrm{MHz}, \mathrm{CDCl}_{3}-\mathrm{TMS}\right)$ $\delta$, ppm: $0.80(\mathrm{~d}, J=8.0 \mathrm{~Hz}, 6 \mathrm{H}), 0.83-0.99(\mathrm{~m}, 14 \mathrm{H}), 1.00-1.16(\mathrm{~m}, 4 \mathrm{H}), 1.29-1.46(\mathrm{~m}, 8 \mathrm{H}), 1.55-1.78(\mathrm{~m}, 12 \mathrm{H}), 1.90-2.02(\mathrm{~m}, 4 \mathrm{H}), 2.11-2.28(\mathrm{~m}, 4 \mathrm{H}), 2.36(\mathrm{t}, J=8.0 \mathrm{~Hz}, 4 \mathrm{H})$, 3.37-3.55 (m, 4H), 3.56-3.64 (ddd, $\left.J_{1}=4.0 \mathrm{~Hz}, J_{2}=4.0 \mathrm{~Hz}, J_{3}=4.0 \mathrm{~Hz}, 2 \mathrm{H}\right), 4.33-4.48(\mathrm{~m}, 4 \mathrm{H}), 5.08-5.31(\mathrm{~m}, 6 \mathrm{H}), 5.79(\mathrm{~s}, 2 \mathrm{H}), 7.66(\mathrm{~s}, 2 \mathrm{H}){ }^{13} \mathrm{C} \mathrm{NMR}\left(100 \mathrm{MHz}, \mathrm{CDCl}{ }_{3}-\right.$ TMS) $\delta$, ppm: 15.9, 21.1, 22.1, 22.8, 24.2, 25.5, 25.7, 27.1, 30.2, 31.6, 33.7, 33.9, 42.4, 43.6, 48.1, 49.4, 57.4, 77.3, 81.8, 98.5, 123.9, 142.8, 159.8, 168.0, 173.2; IR (neat) v, $\mathrm{cm}^{-1}: 3313,3143,3093,2951,2923,2868,1742,1646,1523,1457,1317,1155,1122,958,631$; ESI-MS $m / z(\%): 1109$ ([M+H] $\left.]^{+}, 40\right), 1131$ ([M+Na] $\left.]^{+}, 100\right) ;$ Anal. Calcd for $\mathrm{C}_{50} \mathrm{H}_{76} \mathrm{Br}_{2} \mathrm{~N}_{8} \mathrm{O}_{10}$ : C 54.15, H 6.91, N 10.10, Found: C 54.12, H 6.99, N 10.14.

$(R, S, R, S)-(1,1$ '-(butane-1,4-diyl)bis(1H-1,2,3-triazole-4,1-diyl))bis(methylene) bis(4-((S)-4-bromo-2-((1R,2S,5R)-2-isopropyl-5-methylcyclohexyloxy)-5-oxo-2,5-dihydrofuran-3-ylamino)butanoate) (2d). Yellowish oil, yield $216 \mathrm{mg}(41 \%) ;[\alpha]_{\mathrm{D}}{ }^{20}+50.0\left(c 0.020, \mathrm{CH}_{2} \mathrm{Cl}_{2}\right) ; \mathrm{UV}^{-\mathrm{vis}}\left(\mathrm{CH}_{2} \mathrm{Cl}_{2}\right) \lambda_{\max }: 273 \mathrm{~nm} ;{ }^{1} \mathrm{H} \mathrm{NMR}\left(400 \mathrm{MHz}, \mathrm{CDCl}_{3}-\mathrm{TMS}\right)$ $\delta$, ppm: $0.79(\mathrm{~d}, J=8.0 \mathrm{~Hz}, 3 \mathrm{H}), 0.81-0.98(\mathrm{~m}, 14 \mathrm{H}), 0.99-1.16(\mathrm{~m}, 4 \mathrm{H}), 1.28-1.43(\mathrm{~m}, 4 \mathrm{H}), 1.58-1.74(\mathrm{~m}, 4 \mathrm{H}), 1.84-2.05(\mathrm{~m}, 8 \mathrm{H}), 2.09-2.28(\mathrm{~m}, 4 \mathrm{H}), 2.47(\mathrm{t}, J=8.0 \mathrm{~Hz}, 4 \mathrm{H})$, 3.40-3.72 (m, 6H), 4.30-4.51 (m, 4H), $5.23(\mathrm{~s}, 4 \mathrm{H}), 5.45(\mathrm{~s}, 2 \mathrm{H}), 5.78(\mathrm{~s}, 2 \mathrm{H}), 7.66(\mathrm{~s}, 2 \mathrm{H}) ;{ }^{13} \mathrm{C} \mathrm{NMR}\left(100 \mathrm{MHz}, \mathrm{CDCl}_{3}-\mathrm{TMS}\right) \delta, \mathrm{ppm}: 15.8,21.1,22.2,22.7,25.3,25.4,27.1$, 31.0, 31.6, 33.9, 42.4, 43.2, 48.1, 49.4, 57.8, 77.3, 81.8, 98.6, 123.9, 142.5, 159.7, 167.9, 172.9; IR (neat) v, $\mathrm{cm}^{-1}: 3319,3137,3088,2956,2929,2868,1742,1646,1547,1457$, 1322, 1163, 1125, 952, 628; ESI-MS m/z (\%): 1053 ([M+H $\left.]^{+}, 55\right), 1075$ ([M+Na $\left.]^{+}, 100\right)$; Anal. Calcd for $\mathrm{C}_{46} \mathrm{H}_{68} \mathrm{Br}_{2} \mathrm{~N}_{8} \mathrm{O}_{10}$ : C 52.47, H 6.51, N 10.64, Found: C 52.50, H 6.57, N 10.60. 
(S,S,R,S,2S,2'S)-(1,1'-(butane-1,4-diyl)bis(1H-1,2,3-triazole-4,1-diyl))bis(methylene) bis(2-((S)-4-bromo-5-oxo-2-((1S,2R,4S)-1,7,7-trimethylbi-cyclo[2.2.1]heptan2-yloxy)-2,5-dihydrofuran-3-ylamino)-3-methylbutanoate) (2e). Yellowish oil, yield $288 \mathrm{mg}(49 \%) ;[\alpha]_{\mathrm{D}}{ }^{20}+77.6\left(c 0.077, \mathrm{CH}_{2} \mathrm{Cl}_{2}\right) ; \mathrm{UV}^{-\mathrm{vis}}\left(\mathrm{CH}_{2} \mathrm{Cl}_{2}\right) \lambda_{\text {max }}: 270 \mathrm{~nm}$; ${ }^{1} \mathrm{H}$ NMR (400 MHz, $\mathrm{CDCl}_{3}$-TMS) $\delta$, ppm: 0.68-1.10 (m, 30H), 1.12-1.35 (m, 8H), 1.66-1.82 (m, 4H), 1.89-2.03 (m, 4H), 2.19-2.32 (m, 4H), 3.98 (d, $\left.J=8.0 \mathrm{~Hz}, 2 \mathrm{H}\right), 4.36-4.48$ (m, 4H), $4.75(\mathrm{~b}, 2 \mathrm{H}), 5.23-5.42(\mathrm{~m}, 6 \mathrm{H}), 5.72(\mathrm{~s}, 2 \mathrm{H}), 7.71(\mathrm{~s}, 2 \mathrm{H}) ;{ }^{13} \mathrm{C} \mathrm{NMR}\left(100 \mathrm{MHz}, \mathrm{CDCl}_{3}-\mathrm{TMS}\right) \delta$, ppm: 13.9, 17.3, 18.4, 18.8, 19.6, 26.5, 27.1, 27.9, 32.0, 37.0, 44.8, $47.6,49.4,49.5,58.5,60.4,77.3,88.1,99.8,124.2,141.9,157.9,167.6,170.6$; IR (neat) $v, \mathrm{~cm}^{-1}: 3299,3138,3071,2956,2929,2874,1742,1651,1534,1456,1329,1194$, 1135, 956, 647; ESI-MS m/z (\%): 1077 ([M+H] $\left.]^{+}, 48\right), 1099$ ([M+Na] $\left.]^{+}, 100\right)$; Anal. Calcd for $\mathrm{C}_{48} \mathrm{H}_{68} \mathrm{Br}_{2} \mathrm{~N}_{8} \mathrm{O}_{10}$ : C 53.53, H 6.36, N 10.41, Found: C 53.56, H 6.40, N 10.38.

(S,S,R,S,2S,2'S)-(1,1'-(butane-1,4-diyl)bis(1H-1,2,3-triazole-4,1-diyl))bis(methylene) bis(2-((S)-4-bromo-5-oxo-2-((1S,2R,4S)-1,7,7-trimethylbicyclo[2.2.1]heptan2-yloxy)-2,5-dihydrofuran-3-ylamino)-3-phenylpropanoate) (2f). Yellowish oil, yield $246 \mathrm{mg}(42 \%) ;[\alpha]_{\mathrm{D}}{ }^{20}+77.7\left(c 0.026, \mathrm{CH}_{2} \mathrm{Cl}_{2}\right) ; \mathrm{UV}^{2} \mathrm{vis}\left(\mathrm{CH}_{2} \mathrm{Cl}_{2}\right) \lambda_{\text {max }}: 271 \mathrm{~nm}$; ${ }^{1} \mathrm{H}$ NMR (400 MHz, $\mathrm{CDCl}_{3}$-TMS) $\delta$, ppm: 0.70-0.97 (m, 18H), 1.14-1.29 (m, 8H), 1.62-1.73 (m, 4H), 1.88-2.01 (m, 4H), 2.10-2.23 (m, 2H), 2.97-3.26 (m, 4H), 3.73-3.90 (m, 2H), 4.33-4.48 (m, 4H), $4.86(\mathrm{~b}, 2 \mathrm{H})$, 5.11-5.53 (m, 8H), 7.03-7.16 (m, 4H), 7.24-7.31 (m, 6H), 7.61 (s, 2H); ${ }^{13} \mathrm{C} \mathrm{NMR}\left(100 \mathrm{MHz}, \mathrm{CDCl} \mathrm{N}_{3}-\mathrm{TMS}\right) \delta$, ppm: 14.0, 18.8, 19.6, 26.5, 27.1, 27.9, 36.9, 39.4, 44.8, 47.6, 49.4, 49.4, 56.8, 58.8, 77.3, 88.1, 99.4, 124.5, 127.8, 129.0, 129.4, 134.1, 141.8, 156.7, 167.4, 170.3; IR (neat) v, cm ${ }^{-1}: 3380,3137,3060,3027$, 2952, 2926, 2874, 1747, 1646, 1520, 1497, 1455, 1323, 1178, 1130, 956, 743, 702, 647; ESI-MS m/z (\%): 1173 ([M+H] $\left.]^{+}, 25\right), 1195$ ([M+Na $\left.]^{+}, 100\right)$; Anal. Calcd for $\mathrm{C}_{56} \mathrm{H}_{68} \mathrm{Br}_{2} \mathrm{~N}_{8} \mathrm{O}_{10}$ : C 57.34, H 5.84, N 9.55, Found: C 57.37, H 5.80, N 9.53.

$(S, S, R, S)-(1,1$ '-(butane-1,4-diyl)bis(1H-1,2,3-triazole-4,1-diyl))bis(methylene) ～bis(6-((S)-4-bromo-5-oxo-2-((1S,2R,4S)-1,7,7-trimethylbicyclo[2.2.1]heptan-2-yloxy)-2,5-dihydrofuran-3-ylamino)hexanoate) (2g). Yellowish oil, yield $238 \mathrm{mg}(43 \%) ;[\alpha]_{\mathrm{D}}{ }^{20}+45.0\left(c 0.027, \mathrm{CH}_{2} \mathrm{Cl}_{2}\right) ; \mathrm{UV}^{-\mathrm{vis}}\left(\mathrm{CH}_{2} \mathrm{Cl}{ }_{2}\right) \lambda_{\text {max }}: 273 \mathrm{~nm}$; ${ }^{1} \mathrm{H} \mathrm{NMR}(400 \mathrm{MHz}$, $\left.\mathrm{CDCl}_{3}-\mathrm{TMS}\right) \delta$, ppm: $0.86(\mathrm{~s}, 12 \mathrm{H}), 0.89(\mathrm{~s}, 6 \mathrm{H}), 1.19-1.30(\mathrm{~m}, 8 \mathrm{H}), 1.35-1.45(\mathrm{~m}, 4 \mathrm{H}), 1.63-1.87(\mathrm{~m}, 12 \mathrm{H}), 1.91-2.00(\mathrm{~m}, 4 \mathrm{H}), 2.19-2.29(\mathrm{~m}, 2 \mathrm{H}), 2.36(\mathrm{t}, J=8.0 \mathrm{~Hz}, 4 \mathrm{H})$, 3.37-3.56 (m, 4H), $3.99(\mathrm{~d}, J=8.0 \mathrm{~Hz}, 2 \mathrm{H}), 4.35-4.46(\mathrm{~m}, 4 \mathrm{H}), 5.20(\mathrm{~s}, 4 \mathrm{H}), 5.30-5.39(\mathrm{~m}, 2 \mathrm{H}), 5.77(\mathrm{~s}, 2 \mathrm{H}), 7.64(\mathrm{~s}, 2 \mathrm{H}) ;{ }^{13} \mathrm{C} \mathrm{NMR}\left(100 \mathrm{MHz}, \mathrm{CDCl}{ }_{3}-\mathrm{TMS}\right) \delta, \mathrm{ppm}: 14.0$, 18.8, 19.6, 24.2, 25.8, 26.6, 27.1, 28.0, 30.2, 33.7, 37.1, 44.0, 44.8, 47.6, 49.4, 49.4, 57.4, 77.3, 87.5, 99.1, 123.8, 142.9, 159.9, 167.9, 173.2; IR (neat) v, cm ${ }^{-1}: 3288,3137$,

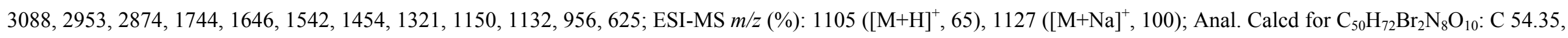
H 6.57, N 10.14, Found: C 54.31, H 6.60, N 10.18.

$(S, S, R, S)-(1,1$ '-(butane-1,4-diyl)bis(1H-1,2,3-triazole-4,1-diyl))bis(methylene) $\quad$ bis(4-((S)-4-bromo-5-oxo-2-((1S,2R,4S)-1,7,7-trimethylbicyclo[2.2.1]heptan-2-yloxy)-2,5-dihydrofuran-3-ylamino)butanoate) (2h). Yellowish oil, yield $\left.241 \mathrm{mg}(46 \%) ;[\alpha]_{\mathrm{D}}{ }^{20}+98.6(c) 0.014, \mathrm{CH}_{2} \mathrm{Cl}_{2}\right) ; \mathrm{UV}^{-v i s}\left(\mathrm{CH}_{2} \mathrm{Cl}_{2}\right) \lambda_{\max }: 273 \mathrm{~nm}$; ${ }^{1} \mathrm{H} \mathrm{NMR}(400 \mathrm{MHz}$, $\left.\mathrm{CDCl}_{3}-\mathrm{TMS}\right) \delta$, ppm: $0.86(\mathrm{~s}, 12 \mathrm{H}), 0.88(\mathrm{~s}, 6 \mathrm{H}), 1.20-1.29(\mathrm{~m}, 8 \mathrm{H}), 1.65-1.85(\mathrm{~m}, 4 \mathrm{H}), 1.92-2.03(\mathrm{~m}, 8 \mathrm{H}), 2.20-2.30(\mathrm{~m}, 2 \mathrm{H}), 2.41-2.55(\mathrm{~m}, 4 \mathrm{H}), 3.44-3.62(\mathrm{~m}, 4 \mathrm{H}), 3.99(\mathrm{~d}$, 
$J=8.0 \mathrm{~Hz}, 2 \mathrm{H}), 4.32-4.45(\mathrm{~m}, 4 \mathrm{H}), 5.17-5.31(\mathrm{~m}, 4 \mathrm{H}), 5.54-5.64(\mathrm{~m}, 2 \mathrm{H}), 5.75(\mathrm{~s}, 2 \mathrm{H}), 7.65(\mathrm{~s}, 2 \mathrm{H}) ;{ }^{13} \mathrm{C} \mathrm{NMR}\left(100 \mathrm{MHz}, \mathrm{CDCl}{ }_{3}-\mathrm{TMS}\right) \delta, \mathrm{ppm}: 14.0,18.8,19.6,25.2,26.6$, $27.1,28.0,31.2,37.1,43.5,44.8,47.6,49.4,57.8,77.3,87.6,99.1,123.9,142.6,160.0,167.7,173.0 ;$ IR (neat) $v, \mathrm{~cm}^{-1}: 3290,3136,2953,2926,2874,1740,1645,1561,1457$, 1322, 1165, 1131, 954, 647; ESI-MS m/z (\%); $1049\left([\mathrm{M}+\mathrm{H}]^{+}, 58\right), 1071\left([\mathrm{M}+\mathrm{Na}]^{+}, 100\right)$; Anal. Calcd for $\mathrm{C}_{46} \mathrm{H}_{64} \mathrm{Br}_{2} \mathrm{~N}_{8} \mathrm{O}_{10}$ : C 52.68, H 6.15, N 10.68, Found: C 52.70, H 6.12, N 10.71 .

\section{General procedure for the synthesis of intermediates 3}

A flame-dried flask was charged with $1 \mathrm{mmol} N$-[(5S)-5-alkoxy-2(5H)-furanonyl] amino acid propargyl ester $\mathbf{1}, 2 \mathrm{mmol}$ 1,4-diazidobutane, $0.05 \mathrm{mmol} \mathrm{Cu}(\mathrm{OAc})_{2} \cdot \mathrm{H}_{2} \mathrm{O}$ and $0.1 \mathrm{mmol} \mathrm{Cu}$ in $\mathrm{CH}_{3} \mathrm{CN}(5 \mathrm{~mL})$. The resulting mixture was stirred at room temperature under $\mathrm{N}_{2}$ atmosphere until the monitoring by TLC showed almost complete conversion of the substrate (about $12 \mathrm{~h}$ ). Then, according to the procedure mentioned above, the reaction mixture was treated to yield the desired compounds $3 \mathbf{a}-3 \mathbf{h}$ in $44-63 \%$ isolated yields.

(S)-(1-(4-azidobutyl)-1H-1,2,3-triazol-4-yl)methyl 2-((S)-4-bromo-2-((1R,2S,5R)-2-isopropyl-5-methylcyclohexyloxy)-5-oxo-2,5-dihydrofuran-3-ylamino)-3-methylbutanoate (3a). Yellowish oil, yield $385 \mathrm{mg}(63 \%)$; $[\alpha]_{\mathrm{D}}{ }^{20}+64.3\left(c 0.098, \mathrm{CH}_{2} \mathrm{Cl}_{2}\right)$; UV-vis $\left(\mathrm{CH}_{2} \mathrm{Cl}_{2}\right) \lambda_{\max }: 270 \mathrm{~nm} ;{ }^{1} \mathrm{H} \mathrm{NMR}\left(400 \mathrm{MHz}, \mathrm{CDCl}{ }_{3}-\mathrm{TMS}\right) \delta$, ppm: $0.85(\mathrm{~d}, J=$ $4.0 \mathrm{~Hz}, 3 \mathrm{H}), 0.87-1.02(\mathrm{~m}, 13 \mathrm{H}), 1.03-1.15(\mathrm{~m}, 2 \mathrm{H}), 1.32-1.46(\mathrm{~m}, 2 \mathrm{H}), 1.57-1.75(\mathrm{~m}, 4 \mathrm{H}), 1.98-2.08(\mathrm{~m}, 2 \mathrm{H}), 2.15-2.35(\mathrm{~m}, 3 \mathrm{H}), 3.36(\mathrm{t}, J=8.0 \mathrm{~Hz}, 2 \mathrm{H}), 3.56-3.66\left(\mathrm{ddd}, J_{1}=\right.$ $\left.4.0 \mathrm{~Hz}, J_{2}=4.0 \mathrm{~Hz}, J_{3}=4.0 \mathrm{~Hz}, 1 \mathrm{H}\right), 4.46(\mathrm{t}, J=8.0 \mathrm{~Hz}, 2 \mathrm{H}), 4.82(\mathrm{~b}, 1 \mathrm{H}), 5.29-5.46(\mathrm{~m}, 3 \mathrm{H}), 5.79(\mathrm{~s}, 1 \mathrm{H}), 7.80(\mathrm{~s}, 1 \mathrm{H}) ;{ }^{13} \mathrm{C} \mathrm{NMR}\left(100 \mathrm{MHz}, \mathrm{CDCl}{ }_{3}-\mathrm{TMS}\right) \delta, \mathrm{ppm}: 15.6,17.2$, $17.8,20.8,22.0,22.6,25.7,27.4,31.4,32.1,33.8,42.2,47.8,49.7,50.5,58.5,59.7,77.5,83.1,100.0,124.1,141.7,157.0,167.7,170.3 ;$ IR (neat) v, cm ${ }^{-1}: 3383,3145,3082$, 2956, 2928, 2871, 2097, 1744, 1651, 1528, 1456, 1316, 1195, 1134, 956, 632; ESI-MS m/z (\%): $612\left([\mathrm{M}+\mathrm{H}]^{+}, 67\right), 634$ ([M+Na $\left.]^{+}, 100\right)$; Anal. Calcd for $\mathrm{C}_{26} \mathrm{H}_{40} \mathrm{BrN}_{7} \mathrm{O}_{5}: 51.15$, H 6.60, N 16.06, Found: C 51.20, H 6.62, N 16.02 .

(S)-(1-(4-azidobutyl)-1H-1,2,3-triazol-4-yl)methyl 2-((S)-4-bromo-2-((1R,2S,5R)-2-isopropyl-5-methylcyclohexyloxy)-5-oxo-2,5-dihydrofuran-3-ylamino)-3-phenylpropanoate (3b). Yellowish oil, yield $356 \mathrm{mg}(54 \%)$; $[\alpha]_{\mathrm{D}}{ }^{20}+53.5\left(c 0.114, \mathrm{CH}_{2} \mathrm{Cl}_{2}\right)$; UV-vis $\left(\mathrm{CH}_{2} \mathrm{Cl}_{2}\right) \lambda_{\max }: 271 \mathrm{~nm} ;{ }^{1} \mathrm{H} \mathrm{NMR}\left(400 \mathrm{MHz}, \mathrm{CDCl}{ }_{3}-\mathrm{TMS}\right) \delta$, ppm: $0.72(\mathrm{~d}, J=$ $8.0 \mathrm{~Hz}, 3 \mathrm{H}), 0.75-0.92(\mathrm{~m}, 7 \mathrm{H}), 0.93-1.09(\mathrm{~m}, 2 \mathrm{H}), 1.25-1.40(\mathrm{~m}, 2 \mathrm{H}), 1.54-1.73(\mathrm{~m}, 4 \mathrm{H}), 1.83-2.21(\mathrm{~m}, 4 \mathrm{H}), 3.06-3.27(\mathrm{~m}, 2 \mathrm{H}), 3.35(\mathrm{t}, J=8.0 \mathrm{~Hz}, 2 \mathrm{H}), 3.39-3.52(\mathrm{~m}, 1 \mathrm{H})$, $4.42(\mathrm{t}, J=8.0 \mathrm{~Hz}, 2 \mathrm{H}), 4.81(\mathrm{~b}, 1 \mathrm{H}), 5.19-5.62(\mathrm{~m}, 4 \mathrm{H}), 7.00-7.14(\mathrm{~m}, 2 \mathrm{H}), 7.15-7.33(\mathrm{~m}, 3 \mathrm{H}), 7.65(\mathrm{~s}, 1 \mathrm{H}) ;{ }^{13} \mathrm{C} \mathrm{NMR}\left(100 \mathrm{MHz}, \mathrm{CDCl} \mathrm{NMS}_{3} \delta, \mathrm{ppm}: 15.5,21.0,22.1,22.6\right.$, 25.4, 25.8, 27.5, 31.5, 33.8, 39.9, 42.2, 47.8, 49.8, 50.6, 56.4, 58.9, 77.5, 83.2, 99.2, 124.3, 127.6, 128.7, 129.5, 134.3, 141.6, 156.7, 167.5, 170.1; IR (neat) v, cm ${ }^{-1}: 3376,3137$, 
3055, 3022, 2955, 2927, 2869, 2097, 1749, 1651, 1522, 1495, 1455, 1324, 1183, 1114, 957, 747, 704, 632; ESI-MS m/z (\%): 660 ([M+H], 59$), 682\left([\mathrm{M}+\mathrm{Na}]^{+}, 100\right) ;$ Anal. Calcd for $\mathrm{C}_{30} \mathrm{H}_{40} \mathrm{BrN}_{7} \mathrm{O}_{5}$ : C 54.71, H 6.12, N 14.89, Found: C 54.68, H 6.10, N 14.91.

(1-(4-azidobutyl)-1H-1,2,3-triazol-4-yl)methyl 6-((S)-4-bromo-2-((1R,2S,5R)-2-isopropyl-5-methylcyclohexyloxy)-5-oxo-2,5-dihydrofuran-3-ylamino)hexanoate (3c). Yellowish oil, yield $368 \mathrm{mg}(59 \%) ;[\alpha]_{\mathrm{D}}{ }^{20}+55.6\left(c 0.083, \mathrm{CH}_{2} \mathrm{Cl}_{2}\right)$; UV-vis $\left(\mathrm{CH}_{2} \mathrm{Cl}_{2}\right) \lambda_{\max }: 273 \mathrm{~nm} ;{ }^{1} \mathrm{H} \mathrm{NMR}\left(400 \mathrm{MHz}, \mathrm{CDCl}{ }_{3}-\mathrm{TMS}\right) \delta$, ppm: $0.79(\mathrm{~d}, J=4.0 \mathrm{~Hz}, 3 \mathrm{H})$, 0.82-0.96 (m, 7H), 0.98-1.15 (m, 2H), 1.30-1.44 (m, 4H), 1.57-1.71 (m, 8H), 1.99-2.07 (m, 2H), 2.13-2.26 (m, $2 \mathrm{H}), 2.36(\mathrm{t}, J=8.0 \mathrm{~Hz}, 2 \mathrm{H}), 3.35(\mathrm{t}, J=8.0 \mathrm{~Hz}, 2 \mathrm{H}), 3.40-3.56$ $(\mathrm{m}, 2 \mathrm{H}), 3.57-3.65\left(\mathrm{ddd}, J_{1}=4.0 \mathrm{~Hz}, J_{2}=4.0 \mathrm{~Hz}, J_{3}=4.0 \mathrm{~Hz}, 1 \mathrm{H}\right), 4.42(\mathrm{t}, J=8.0 \mathrm{~Hz}, 2 \mathrm{H}), 5.21(\mathrm{~s}, 2 \mathrm{H}), 5.43(\mathrm{~s}, 1 \mathrm{H}), 5.81(\mathrm{~s}, 1 \mathrm{H}), 7.71(\mathrm{~s}, 1 \mathrm{H}) ;{ }^{13} \mathrm{C} \mathrm{NMR}\left(100 \mathrm{MHz}, \mathrm{CDCl} 3^{-}\right.$ TMS) $\delta$, ppm: 15.8, 21.1, 22.1, 22.7, 24.2, 25.3, 25.7, 25.8, 27.4, 30.1, 31.5, 33.7, 33.9, 42.3, 43.6, 48.0, 49.7, 50.6, 57.4, 77.5, 81.6, 98.3, 123.8, 142.6, 159.9, 168.0, 173.1; IR (neat) $v, \mathrm{~cm}^{-1}: 3240,3137,3099,2951,2926,2868,2096,1733,1650,1550,1456,1315,1158,1126,937,633 ; \mathrm{ESI}-\mathrm{MS} m / z(\%): 626\left([\mathrm{M}+\mathrm{H}]^{+}, 45\right), 648\left([\mathrm{M}+\mathrm{Na}]^{+}, 100\right) ;$ Anal. Calcd for $\mathrm{C}_{27} \mathrm{H}_{42} \mathrm{BrN}_{7} \mathrm{O}_{5}$ : C 51.92, H 6.78, N 15.70, Found: C 51.95, H 6.80, N 15.73.

(1-(4-azidobutyl)-1H-1,2,3-triazol-4-yl)methyl 4-((S)-4-bromo-2-((1R,2S,5R)-2-isopropyl-5-methylcyclohexyloxy)-5-oxo-2,5-dihydrofuran-3-ylamino)butanoate (3d). Yellowish oil, yield $262 \mathrm{mg}(44 \%) ;[\alpha]_{\mathrm{D}}{ }^{20}+55.0\left(c 0.040, \mathrm{CH}_{2} \mathrm{Cl}_{2}\right) ; \mathrm{UV}$-vis $\left(\mathrm{CH}_{2} \mathrm{Cl}_{2}\right) \lambda_{\max }: 272 \mathrm{~nm} ;{ }^{1} \mathrm{H} \mathrm{NMR}\left(400 \mathrm{MHz}, \mathrm{CDCl}{ }_{3}-\mathrm{TMS}\right) \delta$, ppm: $0.79(\mathrm{~d}, J=8.0 \mathrm{~Hz}, 3 \mathrm{H})$, 0.82-0.97 (m, 7H), 0.98-1.13 (m, 2H), 1.29-1.43 (m, 2H), 1.57-1.70 (m, 4H), 1.93-2.06 (m, 4H), 2.10-2.27 (m, 2H), $2.46(\mathrm{t}, J=8.0 \mathrm{~Hz}, 2 \mathrm{H}), 3.35(\mathrm{t}, J=8.0 \mathrm{~Hz}, 2 \mathrm{H}), 3.46-3.65$ $(\mathrm{m}, 3 \mathrm{H}), 4.42(\mathrm{t}, J=8.0 \mathrm{~Hz}, 2 \mathrm{H}), 5.23(\mathrm{~s}, 2 \mathrm{H}), 5.44(\mathrm{~s}, 1 \mathrm{H}), 5.78(\mathrm{~s}, 1 \mathrm{H}), 7.67(\mathrm{~s}, 1 \mathrm{H}) ;{ }^{13} \mathrm{C} \mathrm{NMR}\left(100 \mathrm{MHz}, \mathrm{CDCl}{ }_{3}-\mathrm{TMS}\right) \delta, \mathrm{ppm}: 15.8,21.1,22.1,22.7,25.3,25.4,25.8,27.5$, 31.0, 31.6, 33.9, 42.4, 43.1, 48.1, 49.8, 50.6, 57.8, 77.4, 81.7, 98.5, 123.7, 142.4, 159.5, 167.9, 172.8; IR (neat) v, $\mathrm{cm}^{-1}: 3290,3132,2951,2921,2868,2097,1741,1646,1572$, 1455, 1309, 1164, 1121, 954, 632; ESI-MS m/z (\%): $596\left([\mathrm{M}+\mathrm{H}]^{+}, 80\right), 618\left([\mathrm{M}+\mathrm{Na}]^{+}, 100\right)$; Anal. Calcd for $\mathrm{C}_{25} \mathrm{H}_{38} \mathrm{BrN}_{7} \mathrm{O}_{5}: \mathrm{C}_{50.34} \mathrm{H} 6.42, \mathrm{~N} 16.44$, Found: C 50.32, H 6.39, N 16.43 .

(S)-(1-(4-azidobutyl)-1H-1,2,3-triazol-4-yl)methyl 2-((S)-4-bromo-5-oxo-2-((1S,2R,4S)-1,7,7-trimethylbicyclo[2.2.1]heptan-2-yloxy)-2,5-dihydrofuran-3-ylamino)3-methylbutanoate (3e). Yellowish oil, yield $511 \mathrm{mg}(64 \%)$; $[\alpha]_{\mathrm{D}}{ }^{20}+70.7\left(c 0.069, \mathrm{CH}_{2} \mathrm{Cl}_{2}\right)$; UV-vis $\left(\mathrm{CH}_{2} \mathrm{Cl}_{2}\right) \lambda_{\max }: 270 \mathrm{~nm} ;{ }^{1} \mathrm{H} \mathrm{NMR}\left(400 \mathrm{MHz}, \mathrm{CDCl}{ }_{3}-\mathrm{TMS}\right) \delta$, ppm: 0.60 $1.09(\mathrm{~m}, 15 \mathrm{H}), 1.10-1.47(\mathrm{~m}, 4 \mathrm{H}), 1.58-1.79(\mathrm{~m}, 4 \mathrm{H}), 1.95-2.10(\mathrm{~m}, 2 \mathrm{H}), 2.16-2.34(\mathrm{~m}, 2 \mathrm{H}), 3.36(\mathrm{t}, J=8.0 \mathrm{~Hz}, 2 \mathrm{H}), 3.99(\mathrm{~d}, J=8.0 \mathrm{~Hz}, 1 \mathrm{H}), 4.44(\mathrm{t}, J=8.0 \mathrm{~Hz}, 2 \mathrm{H}), 4.82(\mathrm{~b}$, 1H), 5.25-5.49 (m, 3H), 5.74 (s, 1H), 7.75 (s, 1H); ${ }^{13} \mathrm{C}$ NMR (100 MHz, $\mathrm{CDCl}_{3}$-TMS) $\delta$, ppm: 13.9, 17.3, 18.3, 18.7, 19.5, 25.7, 26.4, 27.4, 27.9, 32.1, 37.0, 44.8, 47.5, 49.4, $49.7,50.6,58.5,60.3,77.5,88.0,99.4,124.1,141.8,157.1,167.2,170.5$; IR (neat) $v, \mathrm{~cm}^{-1}: 3385,3132,3060,2955,2874,2098,1747,1649,1558,1451,1312,1135,956,626$; ESI-MS m/z (\%): $610\left([\mathrm{M}+\mathrm{H}]^{+}, 53\right), 632\left([\mathrm{M}+\mathrm{Na}]^{+}, 100\right)$; Anal. Calcd for $\mathrm{C}_{26} \mathrm{H}_{38} \mathrm{BrN}_{7} \mathrm{O}_{5}$ : C 51.32, H 6.29, N 16.11, Found: C 51.30, H 6.26, N 16.14. 
(S)-(1-(4-azidobutyl)-1H-1,2,3-triazol-4-yl)methyl 2-((S)-4-bromo-5-oxo-2-((1S,2R,4S)-1,7,7-trimethylbicyclo[2.2.1]heptan-2-yloxy)-2,5-dihydrofuran-3-ylamino)3-phenylpropanoate (3f). Yellowish oil, yield $348 \mathrm{mg}(53 \%)$; $[\alpha]_{\mathrm{D}}{ }^{20}+87.5\left(c 0.048, \mathrm{CH}_{2} \mathrm{Cl}_{2}\right)$; UV-vis $\left(\mathrm{CH}_{2} \mathrm{Cl}_{2}\right) \lambda_{\max }: 271 \mathrm{~nm}$; ${ }^{1} \mathrm{H} \mathrm{NMR}\left(400 \mathrm{MHz}, \mathrm{CDCl}{ }_{3}-\mathrm{TMS}\right) \delta$, ppm: 0.83 $1.00(\mathrm{~m}, 9 \mathrm{H}), 1.03-1.37(\mathrm{~m}, 4 \mathrm{H}), 1.57-1.75(\mathrm{~m}, 4 \mathrm{H}), 1.96-2.09(\mathrm{~m}, 2 \mathrm{H}), 2.10-2.23(\mathrm{~m}, 1 \mathrm{H}), 2.96-3.26(\mathrm{~m}, 2 \mathrm{H}), 3.35(\mathrm{t}, J=8.0 \mathrm{~Hz}, 2 \mathrm{H}), 3.67-3.91(\mathrm{~m}, 1 \mathrm{H}), 4.42(\mathrm{t}, J=8.0 \mathrm{~Hz}$ 2H), $4.82(\mathrm{~b}, 1 \mathrm{H}), 5.08-5.55(\mathrm{~m}, 4 \mathrm{H}), 6.99-7.16(\mathrm{~m}, 2 \mathrm{H}), 7.17-7.37(\mathrm{~m}, 3 \mathrm{H}), 7.63(\mathrm{~s}, 1 \mathrm{H}) ;{ }^{13} \mathrm{C} \mathrm{NMR}\left(100 \mathrm{MHz}, \mathrm{CDCl}_{3}-\mathrm{TMS}\right) \delta, \mathrm{ppm}: 14.0,18.7,19.6,25.8,26.5,27.5,27.9$, $36.9,39.8,44.8,47.5,49.4,49.8,50.6,57.6,58.9,77.4,88.1,99.5,124.2,127.7,128.9,129.4,134.8,141.7,156.3,167.0,170.3 ;$ IR (neat) v, cm ${ }^{-1}: 3299,3137,3055,3027$, 2954, 2923, 2874, 2098, 1752, 1651, 1561, 1520, 1495, 1455, 1323, 1179, 1132, 957, 746, 704, 633; ESI-MS m/z (\%): 656 ([M+H $\left.]^{+}, 55\right), 678$ ([M+Na $\left.]^{+}, 100\right)$; Anal. Calcd for $\mathrm{C}_{30} \mathrm{H}_{38} \mathrm{BrN}_{7} \mathrm{O}_{5}$ : C 54.88, H 5.83, N 14.93, Found: C 54.92, H 5.80, N 14.91.

(1-(4-azidobutyl)-1H-1,2,3-triazol-4-yl)methyl 6-((S)-4-bromo-5-oxo-2-((1S,2R,4S)-1,7,7-trimethylbicyclo[2.2.1]heptan-2-yloxy)-2,5-dihydrofuran-3-ylamino)hexanoate (3g). Yellowish oil, yield $379 \mathrm{mg}(61 \%) ;[\alpha]_{\mathrm{D}}{ }^{20}+63.3\left(c 0.111, \mathrm{CH}_{2} \mathrm{Cl}_{2}\right)$; UV-vis $\left(\mathrm{CH}_{2} \mathrm{Cl}_{2}\right) \lambda_{\max }: 274 \mathrm{~nm} ;{ }^{1} \mathrm{H} \mathrm{NMR}\left(400 \mathrm{MHz}, \mathrm{CDCl}{ }_{3}-\mathrm{TMS}\right) \delta$, ppm: $0.86(\mathrm{~s}, 6 \mathrm{H}), 0.89$ $(\mathrm{s}, 3 \mathrm{H}), 1.12-1.33(\mathrm{~m}, 4 \mathrm{H}), 1.34-1.46(\mathrm{~m}, 2 \mathrm{H}), 1.59-1.90(\mathrm{~m}, 8 \mathrm{H}), 1.98-2.07(\mathrm{~m}, 2 \mathrm{H}), 2.19-2.29(\mathrm{~m}, 1 \mathrm{H}), 2.36(\mathrm{t}, J=8.0 \mathrm{~Hz}, 2 \mathrm{H}), 3.35(\mathrm{t}, J=8.0 \mathrm{~Hz}, 2 \mathrm{H}), 3.39-3.66(\mathrm{~m}, 2 \mathrm{H})$, $3.99(\mathrm{~d}, J=8.0 \mathrm{~Hz}, 1 \mathrm{H}), 4.43(\mathrm{t}, J=8.0 \mathrm{~Hz}, 2 \mathrm{H}), 5.21(\mathrm{~s}, 2 \mathrm{H}), 5.66(\mathrm{~s}, 1 \mathrm{H}), 5.79(\mathrm{~s}, 1 \mathrm{H}), 7.72(\mathrm{~s}, 1 \mathrm{H}) ;{ }^{13} \mathrm{C} \mathrm{NMR}\left(100 \mathrm{MHz}, \mathrm{CDCl}{ }_{3}-\mathrm{TMS}\right) \delta, \mathrm{ppm}: 13.9,18.7,19.6,24.2,25.8$, 26.5, 27.4, 27.9, 30.2, 33.7, 37.0, 44.0, 44.8, 47.5, 49.4, 49.7, 50.6, 57.4, 77.5, 87.3, 99.1, 123.8, 142.6, 160.5, 168.0, 173.1; IR (neat) v, $\mathrm{cm}^{-1}: 3315,3143,3088,2951,2875$, 2097, 1741, 1646, 1561, 1455, 1319, 1156, 1132, 955, 647; ESI-MS m/z (\%): $624\left([\mathrm{M}+\mathrm{H}]^{+}, 42\right), 646\left([\mathrm{M}+\mathrm{Na}]^{+}, 100\right)$; Anal. Calcd for $\mathrm{C}_{27} \mathrm{H}_{40} \mathrm{BrN}_{7} \mathrm{O}_{5}: \mathrm{C} 52.09, \mathrm{H}$ 6.48, $\mathrm{N} 15.75$, Found: C 52.11, H 6.47, N 15.78 .

(1-(4-azidobutyl)-1H-1,2,3-triazol-4-yl)methyl 4-((S)-4-bromo-5-oxo-2-((1S,2R,4S)-1,7,7-trimethylbicyclo[2.2.1]heptan-2-yloxy)-2,5-dihydrofuran-3-ylamino)butanoate (3h). Yellowish solid, yield $333 \mathrm{mg}(56 \%)$; m.p. 95.1-95.8 ${ }^{\circ} \mathrm{C} ;[\alpha]_{\mathrm{D}}{ }^{20}+78.9\left(c 0.065, \mathrm{CH}_{2} \mathrm{Cl}_{2}\right) ; \mathrm{UV}^{-\mathrm{vis}}\left(\mathrm{CH}_{2} \mathrm{Cl}_{2}\right) \lambda_{\max }: 273 \mathrm{~nm} ;{ }^{1} \mathrm{H} \mathrm{NMR}\left(400 \mathrm{MHz}, \mathrm{CDCl}_{3}-\mathrm{TMS}\right) \delta$, ppm: 0.82-0.87 (m, 6H), $0.88(\mathrm{~s}, 3 \mathrm{H}), 1.16-1.35(\mathrm{~m}, 4 \mathrm{H}), 1.61-1.87(\mathrm{~m}, 4 \mathrm{H}), 1.95-2.08(\mathrm{~m}, 4 \mathrm{H}), 2.19-2.31(\mathrm{~m}, 1 \mathrm{H}), 2.48(\mathrm{t}, J=8.0 \mathrm{~Hz}, 2 \mathrm{H}), 3.35(\mathrm{t}, J=8.0 \mathrm{~Hz}, 2 \mathrm{H}), 3.46-3.64$ $(\mathrm{m}, 2 \mathrm{H}), 4.00(\mathrm{~d}, J=12.0 \mathrm{~Hz}, 1 \mathrm{H}), 4.42(\mathrm{t}, J=8.0 \mathrm{~Hz}, 2 \mathrm{H}), 5.23(\mathrm{~s}, 2 \mathrm{H}), 5.72(\mathrm{~s}, 1 \mathrm{H}), 5.77(\mathrm{~s}, 1 \mathrm{H}), 7.69(\mathrm{~s}, 1 \mathrm{H}) ;{ }^{13} \mathrm{C} \mathrm{NMR}\left(100 \mathrm{MHz}, \mathrm{CDCl}{ }_{3}-\mathrm{TMS}\right) \delta, \mathrm{ppm}: 14.0,18.8,19.6$, 25.3, 25.8, 26.5, 27.5, 28.0, 31.1, 37.0, 43.4, 44.8, 47.5, 49.4, 49.8, 50.6, 57.8, 77.4, 87.5, 99.2, 123.7, 142.4, 160.5, 167.8, 172.9; IR (neat) v, cm ${ }^{-1}: 3319,3138,3093,2954$, 2879, 2098, 1743, 1651, 1531, 1456, 1322, 1167, 1132, 954, 661; ESI-MS m/z (\%): $594\left([\mathrm{M}+\mathrm{H}]^{+}, 33\right), 616$ ([M+Na $\left.]^{+}, 100\right)$; Anal. Calcd for C ${ }_{25} \mathrm{H}_{36} \mathrm{BrN}_{7} \mathrm{O}_{5}$ : C 50.51, H 6.10, N 16.49, Found: C 50.29, H 6.38, N 16.48

\section{General procedure for the synthesis of target compounds 5}


A flame-dried flask was charged with $1 \mathrm{mmol}$ intermediate $3,2 \mathrm{mmol}$ alkyne $4,0.05 \mathrm{mmol} \mathrm{Cu}(\mathrm{OAc})_{2} \bullet \mathrm{H}_{2} \mathrm{O}$ and $0.1 \mathrm{mmol} \mathrm{Cu}$ in $\mathrm{CH}_{3} \mathrm{CN}(5 \mathrm{~mL})$. The resulting mixture was stirred at room temperature under $\mathrm{N}_{2}$ atmosphere until the monitoring by TLC showed almost complete conversion of the substrate (about 2 h). Then, according to the procedure mentioned above, the reaction mixture was treated to yield the desired compounds $\mathbf{5 a a - 5 b h}$ in $57-98 \%$ isolated yields.

\section{(S)-(1-(4-(4-phenyl-1H-1,2,3-triazol-1-yl)butyl)-1H-1,2,3-triazol-4-yl)methyl}

2-((S)-4-bromo-2-((1R,2S,5R)-2-isopropyl-5-methylcyclohexyloxy)-5-oxo-2,5-

dihydro-furan-3-ylamino)-3-methylbutanoate (5aa). Yellowish solid, yield $634 \mathrm{mg}(89 \%) ;$ m.p. $143.5-144.1{ }^{\circ} \mathrm{C} ;[\alpha]_{\mathrm{D}}^{20}+38.2\left(c 0.022, \mathrm{CH}_{2} \mathrm{Cl}_{2}\right) ; \mathrm{UV}^{2}-\mathrm{vis}\left(\mathrm{CH}_{2} \mathrm{Cl}_{2}\right) \lambda_{\text {max }}: 257$ $\mathrm{nm} ;{ }^{1} \mathrm{H}$ NMR (400 MHz, $\mathrm{CDCl}_{3}$-TMS) $\delta$, ppm: $0.82(\mathrm{~d}, J=8.0 \mathrm{~Hz}, 3 \mathrm{H}), 0.84-0.97(\mathrm{~m}, 13 \mathrm{H}), 0.98-1.11(\mathrm{~m}, 2 \mathrm{H}), 1.28-1.42(\mathrm{~m}, 2 \mathrm{H}), 1.59-1.70(\mathrm{~m}, 2 \mathrm{H}), 1.89-2.04(\mathrm{~m}, 4 \mathrm{H})$, 2.11-2.28 (m, 3H), 3.49-3.59 (ddd, $\left.J_{1}=4.0 \mathrm{~Hz}, J_{2}=4.0 \mathrm{~Hz}, J_{3}=4.0 \mathrm{~Hz}, 1 \mathrm{H}\right), 4.33-4.49(\mathrm{~m}, 4 \mathrm{H}), 4.79(\mathrm{~b}, 1 \mathrm{H}), 5.24-5.35(\mathrm{~m}, 3 \mathrm{H}), 5.71(\mathrm{~s}, 1 \mathrm{H}), 7.30-7.44(\mathrm{~m}, 3 \mathrm{H}), 7.70(\mathrm{~s}, 1 \mathrm{H})$, 7.78-7.85 (m, 2H), 7.87 (s, 1H); ${ }^{13} \mathrm{C} \mathrm{NMR} \mathrm{(100} \mathrm{MHz,} \mathrm{CDCl}_{3}$-TMS) $\delta$, ppm: 15.7, 17.3, 17.9, 20.9, 22.1, 22.7, 25.8, 27.1, 27.1, 31.4, 32.0, 33.8, 42.2, 47.8, 49.3, 49.4, 58.5, $59.7,77.4,83.3,100.0,120.0,124.2,125.6,128.2,128.8,130.4,141.8,147.7,157.4,167.9,170.4 ;$ IR (neat) $v, \mathrm{~cm}^{-1}: 3383,3136,3099,3060,2956,2927,2870,1744,1651$, 1556, 1521, 1464, 1316, 1192, 1133, 956, 766, 696, 646; ESI-MS m/z (\%): 714 ([M+H] $\left.]^{+}, 100\right)$; Anal. Calcd for $\mathrm{C}_{34} \mathrm{H}_{46} \mathrm{BrN}_{7} \mathrm{O}_{5}$ : C 57.30, H 6.51, N 13.76, Found: C 57.28, H $6.49, \mathrm{~N} 13.64$

(S)-(1-(4-(4-phenyl-1H-1,2,3-triazol-1-yl)butyl)-1H-1,2,3-triazol-4-yl)methyl

2-((S)-4-bromo-2-((1R,2S,5R)-2-isopropyl-5-methylcyclohexyloxy)-5-oxo-2,5dihydro-furan-3-ylamino)-3-phenylpropanoate (5ab). Yellowish oil, yield $662 \mathrm{mg}(87 \%)$; $[\alpha]_{\mathrm{D}}{ }^{20}+50.3\left(c{ }^{0.095}, \mathrm{CH}_{2} \mathrm{Cl}_{2}\right) ; \mathrm{UV}^{-v i s}\left(\mathrm{CH}_{2} \mathrm{Cl}_{2}\right) \lambda_{\text {max }}: 257 \mathrm{~nm}$; ${ }^{1} \mathrm{H} \mathrm{NMR}(400$ MHz, $\mathrm{CDCl}_{3}$-TMS) $\delta$, ppm: $0.69(\mathrm{~d}, J=4.0 \mathrm{~Hz}, 3 \mathrm{H}), 0.74-0.90(\mathrm{~m}, 7 \mathrm{H}), 0.92-1.05(\mathrm{~m}, 2 \mathrm{H}), 1.23-1.35(\mathrm{~m}, 2 \mathrm{H}), 1.56-1.67(\mathrm{~m}, 2 \mathrm{H}), 1.82-2.13(\mathrm{~m}, 6 \mathrm{H}), 3.06-3.23(\mathrm{~m}, 2 \mathrm{H}), 3.34-$ $3.46(\mathrm{~m}, 1 \mathrm{H}), 4.32-4.47(\mathrm{~m}, 4 \mathrm{H}), 4.79(\mathrm{~b}, 1 \mathrm{H}), 5.10-5.49(\mathrm{~m}, 4 \mathrm{H}), 7.01-7.11(\mathrm{~m}, 2 \mathrm{H}), 7.18-7.28(\mathrm{~m}, 3 \mathrm{H}), 7.29-7.43(\mathrm{~m}, 3 \mathrm{H}), 7.64(\mathrm{~s}, 1 \mathrm{H}), 7.75-7.84(\mathrm{~m}, 2 \mathrm{H}), 7.87(\mathrm{~s}, 1 \mathrm{H}) ;{ }^{13} \mathrm{C}$ NMR (100 MHz, $\mathrm{CDCl}_{3}$-TMS) $\delta$, ppm: 15.5, 21.0, 22.1, 22.6, 25.4, 27.1, 31.5, 33.8, 39.7, 42.2, 47.8, 49.3, 49.4, 55.6, 58.8, 77.5, 83.0, 99.3, 120.1, 124.4, 125.6, 127.6, 128.2, $128.7,128.9,129.5,130.4,134.2,141.6,147.7,156.9,167.6,170.1$; IR (neat) $v, \mathrm{~cm}^{-1}: 3374,3136,3062,3028,2953,2926,2868,1747,1648,1518,1497,1484,1455,1325$, 1182, 1117, 956, 766, 698, 647; ESI-MS m/z (\%): 762 ([M+H] $\left.]^{+}, 100\right), 784$ ([M+Na $\left.]^{+}, 80\right)$; Anal. Calcd for $\mathrm{C}_{38} \mathrm{H}_{46} \mathrm{BrN}_{7} \mathrm{O}_{5}: \mathrm{C} 60.00, \mathrm{H} 6.09, \mathrm{~N} 12.89, \mathrm{Found}$ : C 60.06, H 6.13, N 12.86.

(1-(4-(4-phenyl-1H-1,2,3-triazol-1-yl)butyl)-1H-1,2,3-triazol-4-yl)methyl 6-((S)-4-bromo-2-((1R,2S,5R)-2-isopropyl-5-methylcyclohexyloxy)-5-oxo-2,5-dihydrofuran-3-ylamino)hexanoate (5ac). Yellowish solid, yield $414 \mathrm{mg}(57 \%)$; m.p. $125.6-126.8{ }^{\circ} \mathrm{C} ;[\alpha]_{\mathrm{D}}{ }^{20}+74.0\left(c 0.049, \mathrm{CH}_{2} \mathrm{Cl}_{2}\right)$; $\mathrm{UV}_{-}-\mathrm{vis}\left(\mathrm{CH}_{2} \mathrm{Cl}{ }_{2}\right) \lambda_{\max }: 256 \mathrm{~nm}$; ${ }^{1} \mathrm{H} \mathrm{NMR}(400$ $\left.\mathrm{MHz}, \mathrm{CDCl}_{3}-\mathrm{TMS}\right) \delta$, ppm: $0.77(\mathrm{~d}, J=4.0 \mathrm{~Hz}, 3 \mathrm{H}), 0.80-0.96(\mathrm{~m}, 7 \mathrm{H}), 0.97-1.13(\mathrm{~m}, 2 \mathrm{H}), 1.28-1.41(\mathrm{~m}, 4 \mathrm{H}), 1.56-1.75(\mathrm{~m}, 6 \mathrm{H}), 1.90-2.04(\mathrm{~m}, 4 \mathrm{H}), 2.10-2.24(\mathrm{~m}, 2 \mathrm{H}), 2.33$ $(\mathrm{t}, J=8.0 \mathrm{~Hz}, 2 \mathrm{H}), 3.36-3.52(\mathrm{~m}, 2 \mathrm{H}), 3.53-3.61\left(\mathrm{ddd}, J_{1}=4.0 \mathrm{~Hz}, J_{2}=4.0 \mathrm{~Hz}, J_{3}=4.0 \mathrm{~Hz}, 1 \mathrm{H}\right), 4.33-4.51(\mathrm{~m}, 4 \mathrm{H}), 5.12-5.27(\mathrm{~m}, 3 \mathrm{H}), 5.76(\mathrm{~s}, 1 \mathrm{H}), 7.30-7.44(\mathrm{~m}, 2 \mathrm{H}), 7.63(\mathrm{~s}$, 
1H), 7.77-7.92 (m, 3H); ${ }^{13} \mathrm{C}$ NMR (100 MHz, $\mathrm{CDCl}_{3}$-TMS) $\delta$, ppm: 15.9, 21.1, 22.1, 22.7, 24.2, 25.5, 25.7, 27.1, 27.1, 30.2, 31.6, 33.7, 33.9, 42.4, 43.6, 48.0, 49.4, 49.4, 57.4, $77.4,81.7,98.6,119.9,123.8,125.6,128.2,128.9,130.4,142.8,147.8,159.5,168.0,173.1$; IR (neat) $v, \mathrm{~cm}^{-1}: 3320,3137,3096,3060,2953,2928,2868,1742,1647,1555$, 1525, 1484, 1463, 1321, 1159, 1124, 938, 767, 696, 667; ESI-MS m/z (\%): 728 ([M+H] $\left.]^{+}, 100\right)$; Anal. Calcd for $\mathrm{C}_{35} \mathrm{H}_{48} \mathrm{BrN}_{7} \mathrm{O}_{5}: \mathrm{C}_{57.85}$, H 6.66, N 13.49, Found: C 57.88, H $6.64, \mathrm{~N} 13.51$

(1-(4-(4-phenyl-1H-1,2,3-triazol-1-yl)butyl)-1H-1,2,3-triazol-4-yl)methyl 4-((S)-4-bromo-2-((1R,2S,5R)-2-isopropyl-5-methylcyclohexyloxy)-5-oxo-2,5-dihydrofuran-3-ylamino)butanoate (5ad). Yellowish solid, yield $650 \mathrm{mg}(93 \%)$; m.p. 168.8-169.6 ${ }^{\circ} \mathrm{C} ;[\alpha]_{\mathrm{D}}{ }^{20}+101.0\left(c{ }^{0.035}, \mathrm{CH}_{2} \mathrm{Cl}_{2}\right) ; \mathrm{UV}^{-\mathrm{vis}}\left(\mathrm{CH}_{2} \mathrm{Cl}_{2}\right) \lambda_{\max }: 257 \mathrm{~nm}$; ${ }^{1} \mathrm{H} \mathrm{NMR}(400$ $\mathrm{MHz}, \mathrm{CDCl}_{3}$-TMS) $\delta$, ppm: $0.77(\mathrm{~d}, J=4.0 \mathrm{~Hz}, 3 \mathrm{H}), 0.82-0.95(\mathrm{~m}, 7 \mathrm{H}), 0.96-1.12(\mathrm{~m}, 2 \mathrm{H}), 1.27-1.38(\mathrm{~m}, 2 \mathrm{H}), 1.59-1.69(\mathrm{~m}, 2 \mathrm{H}), 1.89-2.04(\mathrm{~m}, 6 \mathrm{H}), 2.09-2.22(\mathrm{~m}, 2 \mathrm{H}), 2.43$ $(\mathrm{t}, J=8.0 \mathrm{~Hz}, 2 \mathrm{H}), 3.46-3.62(\mathrm{~m}, 3 \mathrm{H}), 4.34-4.48(\mathrm{~m}, 4 \mathrm{H}), 5.20(\mathrm{~s}, 2 \mathrm{H}), 5.39(\mathrm{~s}, 1 \mathrm{H}), 5.74(\mathrm{~s}, 1 \mathrm{H}), 7.30-7.46(\mathrm{~m}, 3 \mathrm{H}), 7.61(\mathrm{~s}, 1 \mathrm{H}), 7.75-7.87(\mathrm{~m}, 3 \mathrm{H}) ;{ }^{13} \mathrm{C} \mathrm{NMR}(100 \mathrm{MHz}$, $\mathrm{CDCl}_{3}$-TMS) $\delta$, ppm: 15.8, 21.1, 22.1, 22.8, 25.3, 25.5, 27.1, 27.1, 31.0, 31.6, 33.9, 42.4, 43.2, 48.1, 49.4, 49.4, 57.8, 77.3, 81.7, 98.5 119.8, 123.7, 125.6, 128.2, 128.9, 130.4, 142.6, 147.8, 159.8, 167.8, 172.8; IR (neat) $v, \mathrm{~cm}^{-1}: 3331,3137,3093,3027,2954,2923,2852,1742,1646,1536,1484,1463,1322,1165,1122,951,766,697,646$; ESI-MS $\left.m / z(\%): 700\left([\mathrm{M}+\mathrm{H}]^{+}, 55\right), 722[\mathrm{M}+\mathrm{Na}]^{+}, 100\right)$; Anal. Calcd for $\mathrm{C}_{33} \mathrm{H}_{44} \mathrm{BrN}_{7} \mathrm{O}_{5}: \mathrm{C} 56.73, \mathrm{H} 6.35, \mathrm{~N}$ 14.03, Found: C 56.57, H 6.63, N 14.25.

(S)-(1-(4-(4-phenyl-1H-1,2,3-triazol-1-yl)butyl)-1H-1,2,3-triazol-4-yl)methyl 2-((S)-4-bromo-5-oxo-2-((1S,2R,4S)-1,7,7-trimethylbicyclo[2.2.1]heptan-2-yloxy)-2,5dihydrofuran-3-ylamino)-3-methylbutanoate (5ae). Yellowish oil, yield $696 \mathrm{mg}(98 \%)$; $[\alpha]_{\mathrm{D}}{ }^{20}+38.0\left(c 0.108, \mathrm{CH}_{2} \mathrm{Cl}_{2}\right)$; $\mathrm{UV}_{-\mathrm{vis}}\left(\mathrm{CH}_{2} \mathrm{Cl}_{2}\right) \lambda_{\max }: 256 \mathrm{~nm}$; ${ }^{1} \mathrm{H} \mathrm{NMR}(400 \mathrm{MHz}$, $\mathrm{CDCl}_{3}$-TMS) $\delta$, ppm: $0.82(\mathrm{~s}, 6 \mathrm{H}), 0.87(\mathrm{~s}, 3 \mathrm{H}), 0.89-0.99(\mathrm{~m}, 6 \mathrm{H}), 1.10-1.31(\mathrm{~m}, 4 \mathrm{H}), 1.61-1.77(\mathrm{~m}, 2 \mathrm{H}), 1.89-2.02(\mathrm{~m}, 4 \mathrm{H}), 2.15-2.27(\mathrm{~m}, 2 \mathrm{H}), 3.96(\mathrm{~d}, J=12.0 \mathrm{~Hz}, 1 \mathrm{H})$, 4.34-4.45 (m, 4H), 4.59 (b, 1H), 5.27-5.33 (m, 2H), $5.46(\mathrm{~d}, J=12.0 \mathrm{~Hz}, 1 \mathrm{H}), 5.72(\mathrm{~s}, 1 \mathrm{H}), 7.29-7.43(\mathrm{~m}, 2 \mathrm{H}), 7.72(\mathrm{~s}, 1 \mathrm{H}), 7.77-7.84(\mathrm{~m}, 2 \mathrm{H}), 7.89(\mathrm{~s}, 1 \mathrm{H})$; ${ }^{13} \mathrm{C} \mathrm{NMR}(100$ MHz, $\mathrm{CDCl}_{3}$-TMS) $\delta$, ppm: 13.8, 17.3, 18.3, 18.7, 19.5, 26.4, 27.0, 27.0, 27.9, 32.0, 36.9, 44.8, 47.5, 49.3, 49.4, 58.5, 60.3, 77.5, 87.9, 99.6, 120.0, 124.2, 125.5, 128.1, 128.8, 130.5, 141.8, 147.6, 158.1, 167.5, 170.4; IR (neat) $v, \mathrm{~cm}^{-1}: 3383,3136,3063,3027,2956,2930,2876,1752,1651,1523,1465,1331,1193,1136,957,766,696,661$; ESI-MS $m / z(\%): 710\left([\mathrm{M}+\mathrm{H}]^{+}, 100\right), 732\left([\mathrm{M}+\mathrm{Na}]^{+}, 57\right)$; Anal. Calcd for $\mathrm{C}_{34} \mathrm{H}_{44} \mathrm{BrN}_{7} \mathrm{O}_{5}: \mathrm{C}$ 57.46, H 6.24, N 13.80, Found: C 57.51, H 6.27, N 13.82.

(S)-(1-(4-(4-phenyl-1H-1,2,3-triazol-1-yl)butyl)-1H-1,2,3-triazol-4-yl)methyl 2-((S)-4-bromo-5-oxo-2-((1S,2R,4S)-1,7,7-trimethylbicyclo[2.2.1]heptan-2-yloxy)-2,5dihydrofuran-3-ylamino)-3-phenylpropanoate (5af). Yellowish oil, yield $713 \mathrm{mg}(94 \%) ;[\alpha]_{\mathrm{D}}{ }^{20}+71.4\left(c{ }^{0.056}, \mathrm{CH}_{2} \mathrm{Cl}_{2}\right) ; \mathrm{UV}^{-\mathrm{vis}}\left(\mathrm{CH}_{2} \mathrm{Cl}_{2}\right) \lambda_{\max }: 257 \mathrm{~nm} ;{ }^{1} \mathrm{H} \mathrm{NMR}(400 \mathrm{MHz}$, $\left.\mathrm{CDCl}_{3}-\mathrm{TMS}\right) \delta$, ppm: $0.80(\mathrm{~s}, 6 \mathrm{H}), 0.82(\mathrm{~s}, 3 \mathrm{H}), 1.11-1.32(\mathrm{~m}, 4 \mathrm{H}), 1.59-1.73(\mathrm{~m}, 2 \mathrm{H}), 1.89-2.03(\mathrm{~m}, 4 \mathrm{H}), 2.07-2.16(\mathrm{~m}, 1 \mathrm{H}), 3.02-3.23(\mathrm{~m}, 2 \mathrm{H}), 3.69-3.86(\mathrm{~m}, 1 \mathrm{H}), 4.32-4.48$ $(\mathrm{m}, 4 \mathrm{H}), 4.94(\mathrm{~b}, 1 \mathrm{H}), 5.12-5.37(\mathrm{~m}, 3 \mathrm{H}), 5.45(\mathrm{~s}, 1 \mathrm{H}), 7.04-7.14(\mathrm{~m}, 2 \mathrm{H}), 7.17-7.28(\mathrm{~m}, 3 \mathrm{H}), 7.29-7.42(\mathrm{~m}, 2 \mathrm{H}), 7.61(\mathrm{~s}, 1 \mathrm{H}), 7.75-7.87(\mathrm{~m}, 3 \mathrm{H}) ;{ }^{13} \mathrm{C} \mathrm{NMR}\left(100 \mathrm{MHz}, \mathrm{CDCl}{ }^{-}\right.$ TMS) $\delta$, ppm: 13.9, 18.7, 19.6, 26.5, 27.1, 27.1, 27.9, 36.9, 39.5, 44.8, 47.5, 49.3, 49.4, 49.4, 56.4, 58.8, 77.4, 88.0, 99.5, 120.0, 124.3, 125.6, 127.7, 128.2, 128.8, 128.9, 129.4, 
130.4, 134.7, 141.8, 147.8, 158.4, 167.4, 170.2; IR (neat) $v, \mathrm{~cm}^{-1}: 3375,3136,3066,3029,2953,2925,2868,1749,1651,1519,1495,1484,1455,1324,1180,1130957,765$, 698, 645; ESI-MS m/z (\%): $760\left([\mathrm{M}+\mathrm{H}]^{+}, 100\right)$; Anal. Calcd for $\mathrm{C}_{38} \mathrm{H}_{44} \mathrm{BrN}_{7} \mathrm{O}_{5}$ : C 60.16, H 5.85, N 12.92, Found: C 60.20, H 5.89, N 12.89.

(1-(4-(4-phenyl-1H-1,2,3-triazol-1-yl)butyl)-1H-1,2,3-triazol-4-yl)methyl 6-((S)-4-bromo-5-oxo-2-((1S,2R,4S)-1,7,7-trimethylbicyclo[2.2.1]heptan-2-yloxy)-2,5-dihydrofuran-3-ylamino)hexanoate (5ag). Yellowish oil, yield $609 \mathrm{mg}(84 \%)$; $[\alpha]_{\mathrm{D}}{ }^{20}+42.4\left(c 0.149, \mathrm{CH}_{2} \mathrm{Cl}_{2}\right)$; $\mathrm{UV}_{-\mathrm{vis}}\left(\mathrm{CH}_{2} \mathrm{Cl}_{2}\right) \lambda_{\text {max }}: 256 \mathrm{~nm}$; ${ }^{1} \mathrm{H} \mathrm{NMR}\left(400 \mathrm{MHz}, \mathrm{CDCl}_{3}\right.$ TMS) $\delta$, ppm: 0.71-0.98 (m, 9H), 1.09-1.31 (m, 4H), 1.32-1.43 (m, 2H), 1.61-1.89 (m, 6H), 1.91-2.05 (m, 4H), 2.16-2.27 (m, 1H), 2.32 (t, J= 8.0 Hz, 2H), 3.34-3.56 (m, 2H), $3.97(\mathrm{~d}, J=4.0 \mathrm{~Hz}, 1 \mathrm{H}), 4.33-4.51(\mathrm{~m}, 4 \mathrm{H}), 5.18(\mathrm{~s}, 2 \mathrm{H}), 5.71-5.97(\mathrm{~m}, 2 \mathrm{H}), 7.30-7.47(\mathrm{~m}, 3 \mathrm{H}), 7.69(\mathrm{~s}, 1 \mathrm{H}), 7.75-7.87(\mathrm{~m}, 2 \mathrm{H}), 7.91(\mathrm{~s}, 1 \mathrm{H}) ;{ }^{13} \mathrm{C} \mathrm{NMR}\left(100 \mathrm{MHz}, \mathrm{CDCl}{ }_{3}^{-}\right.$ TMS) $\delta$, ppm: 13.9, 18.7, 19.6, 24.2, 25.7, 26.5, 27.1, 28.0, 30.1, 33.7, 37.0, 44.0, 44.7, 47.5, 49.3, 49.4, 57.4, 77.5, 87.3, 99.1, 120.1, 123.9, 125.6, 128.1, 128.8, 130.4, 142.7, 147.6, 160.5, 168.0, 173.1; IR (neat) $v, \mathrm{~cm}^{-1}: 3319,3136,3095,3060,2952,2874,1744,1648,1555,1525,1459,1320,1155,1131,955,766,696,667 ; \mathrm{ESI}-\mathrm{MS} m / z$ (\%): 726 $\left([\mathrm{M}+\mathrm{H}]^{+}, 100\right), 748\left([\mathrm{M}+\mathrm{Na}]^{+}, 67\right)$; Anal. Calcd for $\mathrm{C}_{35} \mathrm{H}_{46} \mathrm{BrN}_{7} \mathrm{O}_{5}$ : C 58.01, H 6.40, N 13.53, Found: C 58.04, H 6.45, N 13.51.

(1-(4-(4-phenyl-1H-1,2,3-triazol-1-yl)butyl)-1H-1,2,3-triazol-4-yl)methyl 4-((S)-4-bromo-5-oxo-2-((1S,2R,4S)-1,7,7-trimethylbicyclo[2.2.1]heptan-2-yloxy)-2,5-dihydrofuran-3-ylamino)butanoate (5ah). Yellowish solid, yield $691 \mathrm{mg}(97 \%)$; m.p. 106.5-107.4 ${ }^{\circ} \mathrm{C} ;[\alpha]_{\mathrm{D}}{ }^{20}+103.0\left(c 0.045, \mathrm{CH}_{2} \mathrm{Cl}_{2}\right) ; \mathrm{UV}{ }^{-v i s}\left(\mathrm{CH}_{2} \mathrm{Cl}_{2}\right) \lambda_{\text {max }}: 256 \mathrm{~nm} ;{ }^{1} \mathrm{H}$ NMR (400 MHz, $\mathrm{CDCl}_{3}$-TMS) $\delta$, ppm: $0.82(\mathrm{~s}, 6 \mathrm{H}), 0.84(\mathrm{~s}, 3 \mathrm{H}), 1.12-1.36(\mathrm{~m}, 4 \mathrm{H}), 1.62-1.84(\mathrm{~m}, 2 \mathrm{H}), 1.87-2.04(\mathrm{~m}, 6 \mathrm{H}), 2.15-2.27(\mathrm{~m}, 1 \mathrm{H}), 2.44(\mathrm{t}, J=8.0 \mathrm{~Hz}, 2 \mathrm{H}), 3.43-$ $3.63(\mathrm{~m}, 2 \mathrm{H}), 3.96(\mathrm{~d}, J=8.0 \mathrm{~Hz}, 1 \mathrm{H}), 4.33-4.52(\mathrm{~m}, 4 \mathrm{H}), 5.19(\mathrm{~s}, 2 \mathrm{H}), 5.75(\mathrm{~s}, 1 \mathrm{H}), 6.02(\mathrm{~s}, 1 \mathrm{H}), 7.30-7.45(\mathrm{~m}, 3 \mathrm{H}), 7.68(\mathrm{~s}, 1 \mathrm{H}), 7.75-7.84(\mathrm{~m}, 2 \mathrm{H}), 7.90(\mathrm{~s}, 1 \mathrm{H}) ;{ }^{13} \mathrm{C} \mathrm{NMR}$ (100 MHz, CDCl ${ }_{3}$-TMS) $\delta$, ppm: 13.9, 18.8, 19.6, 25.3, 26.5, 27.1, 27.1, 28.0, 31.0, 37.0, 43.4, 44.7, 47.5, 49.4, 49.4, 57.7, 77.5, 87.4, 99.2, 120.1, 123.9, 125.6, 128.2, 128.8, 130.4, 142.5, 147.6, 160.8, 168.1, 172.8; IR (neat) $v \mathrm{~cm}^{-1}: 3312,3136,3093,3049,2954,2929,2874,1743,1646,1556,1531,1463,1322,1165,1131,953,765,696,646$; ESI-MS m/z (\%): $698\left([\mathrm{M}+\mathrm{H}]^{+}, 39\right), 720$ ([M+Na $\left.]^{+}, 100\right)$; Anal. Calcd for $\mathrm{C}_{33} \mathrm{H}_{42} \mathrm{BrN}_{7} \mathrm{O}_{5}$ : C 56.90, H 6.08, N 14.07, Found: C 56.75, H 6.35, N 13.84.

(S)-(1-(4-(4-propyl-1H-1,2,3-triazol-1-yl)butyl)-1H-1,2,3-triazol-4-yl)methyl 2-((S)-4-bromo-2-((1R,2S,5R)-2-isopropyl-5-methylcyclohexyloxy)-5-oxo-2,5dihydro-furan-3-ylamino)-3-methylbutanoate (5ba). Yellowish solid, yield $550 \mathrm{mg}(81 \%) ; \mathrm{m} . \mathrm{p} .120 .6-121.0{ }^{\circ} \mathrm{C} ;[\alpha]_{\mathrm{D}}^{20}+75.7^{\circ}\left(c 0.073, \mathrm{CH}_{2} \mathrm{Cl}_{2}\right) ; \mathrm{UV}^{2}-\mathrm{vis}\left(\mathrm{CH}_{2} \mathrm{Cl}_{2}\right) \lambda_{\max }: 270$ nm; ${ }^{1} \mathrm{H}$ NMR (400 MHz, CDCl 3 -TMS) $\delta$, ppm: 0.84 (d, $\left.J=4.0 \mathrm{~Hz}, 3 \mathrm{H}\right), 0.86-1.01(\mathrm{~m}, 16 \mathrm{H}), 1.02-1.15(\mathrm{~m}, 2 \mathrm{H}), 1.26-1.43(\mathrm{~m}, 2 \mathrm{H}), 1.60-1.77(\mathrm{~m}, 4 \mathrm{H}), 1.87-2.02(\mathrm{~m}, 4 \mathrm{H})$, 2.11-2.31 (m, 3H), $2.68(\mathrm{t}, J=8.0 \mathrm{~Hz}, 2 \mathrm{H}), 3.53-3.64\left(\mathrm{ddd}, J_{1}=4.0 \mathrm{~Hz}, J_{2}=4.0 \mathrm{~Hz}, J_{3}=4.0 \mathrm{~Hz}, 1 \mathrm{H}\right), 4.31-4.46(\mathrm{~m}, 4 \mathrm{H}), 4.81(\mathrm{~b}, 1 \mathrm{H}), 5.24-5.40(\mathrm{~m}, 3 \mathrm{H}), 5.76(\mathrm{~s}, 1 \mathrm{H}), 7.36(\mathrm{~s}$, 1H), 7.70 (s, 1H); ${ }^{13} \mathrm{C}$ NMR (100 MHz, $\mathrm{CDCl}_{3}$-TMS) $\delta$, ppm: 13.8, 15.7, 17.3, 17.9, 20.9, 22.1, 22.6, 22.7, 25.8, 27.1, 27.1, 27.6, 31.5, 32.0, 33.8, 42.2, 47.9, 49.0, 49.4, 58.5, $59.7,77.4,83.2,99.9,120.9,124.1,141.8,148.3,157.3,167.8,170.5$; IR (neat) $v, \mathrm{~cm}^{-1}: 3382,3137,3082,2958,2928,2872,1748,1651,1551,1523,1458,1317,1193,1135$, 956, 661; ESI-MS m/z (\%): $680\left([\mathrm{M}+\mathrm{H}]^{+}, 100\right), 702\left([\mathrm{M}+\mathrm{Na}]^{+}, 67\right)$; Anal. Calcd for $\mathrm{C}_{31} \mathrm{H}_{48} \mathrm{BrN}_{7} \mathrm{O}_{5}$ : C 54.86, H 7.13, N 14.45, Found: C 54.57, H 7.36, N 14.30. 
dihydro-furan-3-ylamino)-3-phenylpropanoate (5bb). Yellowish oil, yield $603 \mathrm{mg}(83 \%)$; $[\alpha]_{\mathrm{D}}{ }^{20}+112.5\left(c{ }^{0.043}, \mathrm{CH}_{2} \mathrm{Cl}_{2}\right) ; \mathrm{UV}^{-v i s}\left(\mathrm{CH}_{2} \mathrm{Cl}_{2}\right) \lambda_{\text {max }}: 271 \mathrm{~nm}$; ${ }^{1} \mathrm{H} \mathrm{NMR}(400$ $\left.\mathrm{MHz}, \mathrm{CDCl}_{3}-\mathrm{TMS}\right) \delta$, ppm: $0.72(\mathrm{~d}, J=8.0 \mathrm{~Hz}, 3 \mathrm{H}), 0.77-1.09(\mathrm{~m}, 12 \mathrm{H}), 1.25-1.39(\mathrm{~m}, 2 \mathrm{H}), 1.60-1.74(\mathrm{~m}, 4 \mathrm{H}), 1.83-2.18(\mathrm{~m}, 6 \mathrm{H}), 2.68(\mathrm{t}, J=8.0 \mathrm{~Hz}, 2 \mathrm{H}), 3.06-3.25(\mathrm{~m}, 2 \mathrm{H})$, 3.37-3.51 (m, 1H), 4.30-4.46 (m, 4H), 5.02-5.58 (m, 5H), 7.03-7.12 (m, 2H), 7.20-7.31 (m, 3H), $7.34(\mathrm{~s}, 1 \mathrm{H}), 7.62(\mathrm{~s}, 1 \mathrm{H}) ;{ }^{13} \mathrm{C} \mathrm{NMR}\left(100 \mathrm{MHz}, \mathrm{CDCl}{ }_{3}-\mathrm{TMS}\right) \delta, \mathrm{ppm}: 13.8$, $15.5,21.0,22.1,22.6,22.7,25.4,27.1,27.6,31.5,33.8,39.7,42.2,47.8,49.0,49.5,56.1,58.8,77.4,82.8,99.8,120.9,124.3,127.6,128.7,129.5,134.2,141.6,148.3,157.6$, 167.6, 170.1; IR (neat) $v \mathrm{~cm}^{-1}: 3379,3137,3088,3029,2956,2928,2870,1751,1649,1552,1519,1497,1456,1325,1183,1120,957,746,704,628 ;$ ESI-MS $m / z$ (\%): 728 $\left([\mathrm{M}+\mathrm{H}]^{+}, 100\right), 750\left([\mathrm{M}+\mathrm{Na}]^{+}, 83\right)$; Anal. Calcd for $\mathrm{C}_{35} \mathrm{H}_{48} \mathrm{BrN}_{7} \mathrm{O}_{5}$ : C 57.85, H 6.66, N 13.49, Found: C 57.82, H 6.70, N 13.47.

(1-(4-(4-propyl-1H-1,2,3-triazol-1-yl)butyl)-1H-1,2,3-triazol-4-yl)methyl 6-((S)-4-bromo-2-((1R,2S,5R)-2-isopropyl-5-methylcyclohexyloxy)-5-oxo-2,5-dihydrofuran-3-ylamino)hexanoate (5bc). Yellowish solid, yield $610 \mathrm{mg}(88 \%)$; m.p. 123.8-124.6 ${ }^{\circ} \mathrm{C} ;[\alpha]_{\mathrm{D}}{ }^{20}+51.3\left(c 0.129, \mathrm{CH}_{2} \mathrm{Cl}_{2}\right) ; \mathrm{UV}^{-v i s}\left(\mathrm{CH}_{2} \mathrm{Cl}_{2}\right) \lambda_{\text {max }}: 272 \mathrm{~nm}$; ${ }^{1} \mathrm{H} \mathrm{NMR}(400$ $\mathrm{MHz}, \mathrm{CDCl}_{3}$-TMS) $\delta$, ppm: $0.79(\mathrm{~d}, J=4.0 \mathrm{~Hz}, 3 \mathrm{H}), 0.84-0.99(\mathrm{~m}, 10 \mathrm{H}), 1.00-1.16(\mathrm{~m}, 2 \mathrm{H}), 1.26-1.42(\mathrm{~m}, 4 \mathrm{H}), 1.59-1.73(\mathrm{~m}, 8 \mathrm{H}), 1.88-2.00(\mathrm{~m}, 4 \mathrm{H}), 2.10-2.26(\mathrm{~m}, 2 \mathrm{H}), 2.36$ $(\mathrm{t}, J=8.0 \mathrm{~Hz}, 2 \mathrm{H}), 2.68(\mathrm{t}, J=8.0 \mathrm{~Hz}, 2 \mathrm{H}), 3.41-3.54(\mathrm{~m}, 2 \mathrm{H}), 3.56-3.63\left(\mathrm{ddd}, J_{1}=4.0 \mathrm{~Hz}, J_{2}=4.0 \mathrm{~Hz}, J_{3}=4.0 \mathrm{~Hz}, 1 \mathrm{H}\right), 4.32-4.44(\mathrm{~m}, 4 \mathrm{H}), 5.15-5.27(\mathrm{~m}, 3 \mathrm{H}), 5.79(\mathrm{~s}, 1 \mathrm{H})$, 7.31 (s, 1H), 7.63 (s, 1H); ${ }^{13} \mathrm{C}$ NMR (100 MHz, $\mathrm{CDCl}_{3}$-TMS) $\delta$, ppm: 13.7, 15.8, 21.0, 22.1, 22.6, 22.6, 24.2, 25.2, 25.7, 27.1, 27.5, 30.1, 31.4, 33.6, 33.8, 42.3, 43.5, 48.0, $49.0,49.3,57.3,77.6,81.3,98.2,120.9,123.8,142.6,148.1,159.6,168.2,173.0 ;$ IR (neat) $v, \mathrm{~cm}^{-1}: 3354,3137,3082,2955,2927,2869,1742,1648,1551,1528,1458,1320$, 1160, 1122, 938, 647; ESI-MS m/z (\%): $694\left([\mathrm{M}+\mathrm{H}]^{+}, 87\right), 716\left([\mathrm{M}+\mathrm{Na}]^{+}, 100\right)$; Anal. Calcd for $\mathrm{C}_{32} \mathrm{H}_{50} \mathrm{BrN}_{7} \mathrm{O}_{5}: \mathrm{C} 55.49, \mathrm{H} 7.28, \mathrm{~N} 14.15, \mathrm{Found}: \mathrm{C} 55.78, \mathrm{H} 7.46, \mathrm{~N} 14.35$

(1-(4-(4-propyl-1H-1,2,3-triazol-1-yl)butyl)-1H-1,2,3-triazol-4-yl)methyl 4-((S)-4-bromo-2-((1R,2S,5R)-2-isopropyl-5-methylcyclohexyloxy)-5-oxo-2,5-dihydrofuran-3-ylamino)butanoate (5bd). Yellowish solid, yield $572 \mathrm{mg}(86 \%) ;$ m.p. $118.2-119.6{ }^{\circ} \mathrm{C} ;[\alpha]_{\mathrm{D}}{ }^{20}+97.7\left(c 0.044, \mathrm{CH}_{2} \mathrm{Cl}_{2}\right) ; \mathrm{UV}$-vis $\left(\mathrm{CH}_{2} \mathrm{Cl}_{2}\right) \lambda_{\max }: 272 \mathrm{~nm} ;{ }^{1} \mathrm{H} \mathrm{NMR}(400$ $\mathrm{MHz}, \mathrm{CDCl}_{3}$-TMS) $\delta$, ppm: $0.77(\mathrm{~d}, J=4.0 \mathrm{~Hz}, 3 \mathrm{H}), 0.80-1.00(\mathrm{~m}, 10 \mathrm{H}), 1.01-1.15(\mathrm{~m}, 2 \mathrm{H}), 1.26-1.40(\mathrm{~m}, 2 \mathrm{H}), 1.58-1.78(\mathrm{~m}, 4 \mathrm{H}), 1.82-2.09(\mathrm{~m}, 6 \mathrm{H}), 2.11-2.27(\mathrm{~m}, 2 \mathrm{H}), 2.46$ $(\mathrm{t}, J=8.0 \mathrm{~Hz}, 2 \mathrm{H}), 2.67(\mathrm{t}, J=8.0 \mathrm{~Hz}, 2 \mathrm{H}), 3.43-3.67(\mathrm{~m}, 3 \mathrm{H}), 4.27-4.50(\mathrm{~m}, 4 \mathrm{H}), 5.22(\mathrm{~s}, 2 \mathrm{H}), 5.74(\mathrm{~s}, 1 \mathrm{H}), 5.81(\mathrm{~s}, 1 \mathrm{H}), 7.35(\mathrm{~s}, 1 \mathrm{H}), 7.67(\mathrm{~s}, 1 \mathrm{H}) ;{ }^{13} \mathrm{C} \mathrm{NMR}(100 \mathrm{MHz}$ $\mathrm{CDCl}_{3}$-TMS) $\delta$, ppm: 13.8, 15.8, 21.1, 22.1, 22.6, 22.7, 25.3, 27.1, 27.1, 27.6, 30.9, 31.5, 33.9, 42.3, 43.1, 48.0, 49.1, 49.4, 57.7, 77.4, 81.6, 98.4, 120.9, 123.8, 142.5, 148.3, 159.8, 168.0, 172.8; IR (neat) $v, \mathrm{~cm}^{-1}: 3334,3137,3077,2956,2926,2870,1743,1651,1552,1525,1458,1321,1165,1122,952,647 ; \mathrm{ESI}-\mathrm{MS} m / z(\%): 666\left([\mathrm{M}+\mathrm{H}]^{+}, 100\right)$, $688\left([\mathrm{M}+\mathrm{Na}]^{+}\right.$, 87); Anal. Calcd for $\mathrm{C}_{30} \mathrm{H}_{46} \mathrm{BrN}_{7} \mathrm{O}_{5}$ : C 54.21, H 6.98, N 14.75, Found: C 54.18, H 6.73, N 14.49.

(S)-(1-(4-(4-propyl-1H-1,2,3-triazol-1-yl)butyl)-1H-1,2,3-triazol-4-yl)methyl 2-((S)-4-bromo-5-oxo-2-((1S,2R,4S)-1,7,7-trimethylbicyclo[2.2.1]heptan-2-yloxy)-2,5dihydrofuran-3-ylamino)-3-methylbutanoate (5be). Yellowish oil, yield $656 \mathrm{mg}(97 \%) ;[\alpha]_{\mathrm{D}}{ }^{20}+84.6\left(c 0.063, \mathrm{CH}_{2} \mathrm{Cl}_{2}\right) ; \mathrm{UV}_{-}$vis $\left(\mathrm{CH}_{2} \mathrm{Cl}_{2}\right) \lambda_{\max }: 270 \mathrm{~nm} ;{ }^{1} \mathrm{H} \mathrm{NMR}(400 \mathrm{MHz}$, 
$\mathrm{CDCl}_{3}$-TMS) $\delta$, ppm: $0.85(\mathrm{~s}, 6 \mathrm{H}), 0.89(\mathrm{~s}, 3 \mathrm{H}), 0.91-1.05(\mathrm{~m}, 9 \mathrm{H}), 1.09-1.33(\mathrm{~m}, 4 \mathrm{H}), 1.62-1.74(\mathrm{~m}, 4 \mathrm{H}), 1.90-2.01(\mathrm{~m}, 4 \mathrm{H}), 2.18-2.31(\mathrm{~m}, 2 \mathrm{H}), 2.67(\mathrm{t}, J=8.0 \mathrm{~Hz}, 2 \mathrm{H}), 3.99$ $(\mathrm{d}, J=8.0 \mathrm{~Hz}, 1 \mathrm{H}), 4.34-4.47(\mathrm{~m}, 4 \mathrm{H}), 4.82(\mathrm{~b}, 1 \mathrm{H}), 5.28-5.39(\mathrm{~m}, 2 \mathrm{H}), 5.53(\mathrm{~d}, J=8.0 \mathrm{~Hz}, 1 \mathrm{H}), 5.77(\mathrm{~s}, 1 \mathrm{H}), 7.39(\mathrm{~s}, 1 \mathrm{H}), 7.75(\mathrm{~s}, 1 \mathrm{H}) ;{ }^{13} \mathrm{C} \mathrm{NMR}\left(100 \mathrm{MHz}, \mathrm{CDCl}{ }_{3}-\mathrm{TMS}\right) \delta$, ppm: $13.7,13.8,17.3,18.3,18.7,19.5,22.6,26.4,27.0,27.1,27.5,27.8,32.0,36.9,44.7,47.5,49.0,49.4,58.4,60.3,77.5,87.9,99.6,120.9,124.2,141.7,148.2,157.6,167.3$, 170.4; IR (neat) $v, \mathrm{~cm}^{-1}: 3286,3136,3079,2958,2931,2874,1752,1649,1552,1523,1458,1331,1192,1136,957,662 ; \mathrm{ESI}-\mathrm{MS} \mathrm{m} / z(\%): 678\left([\mathrm{M}+\mathrm{H}]^{+}, 100\right), 700\left([\mathrm{M}+\mathrm{Na}]^{+}\right.$, 64); Anal. Calcd for $\mathrm{C}_{31} \mathrm{H}_{46} \mathrm{BrN}_{7} \mathrm{O}_{5}$ : C 55.03, H 6.85, N 14.49, Found: C 55.01, H 6.88, N 14.45.

(S)-(1-(4-(4-propyl-1H-1,2,3-triazol-1-yl)butyl)-1H-1,2,3-triazol-4-yl)methyl 2-((S)-4-bromo-5-oxo-2-((1S,2R,4S)-1,7,7-trimethylbicyclo[2.2.1]heptan-2-yloxy)-2,5dihydrofuran-3-ylamino)-3-phenylpropanoate (5bf). Yellowish oil, yield $544 \mathrm{mg}(75 \%) ;[\alpha]_{\mathrm{D}}{ }^{20}+34.3\left(c 0.093, \mathrm{CH}_{2} \mathrm{Cl}_{2}\right) ; \mathrm{UV}^{2}$-vis $\left(\mathrm{CH}_{2} \mathrm{Cl}_{2}\right) \lambda_{\max }: 271 \mathrm{~nm} ;{ }^{1} \mathrm{H} \mathrm{NMR}(400 \mathrm{MHz}$, $\mathrm{CDCl}_{3}$-TMS) $\delta$, ppm: 0.65-0.91 (m, 9H), $0.96(\mathrm{t}, J=8.0 \mathrm{~Hz}, 3 \mathrm{H}), 1.11-1.34(\mathrm{~m}, 4 \mathrm{H}), 1.60-1.74(\mathrm{~m}, 4 \mathrm{H}), 1.87-2.01(\mathrm{~m}, 4 \mathrm{H}), 2.09-2.21(\mathrm{~m}, 1 \mathrm{H}), 2.67(\mathrm{t}, J=8.0 \mathrm{~Hz}, 2 \mathrm{H}), 3.02-$ $3.28(\mathrm{~m}, 2 \mathrm{H}), 3.72-3.91(\mathrm{~m}, 1 \mathrm{H}), 4.30-4.48(\mathrm{~m}, 4 \mathrm{H}), 4.80(\mathrm{~b}, 1 \mathrm{H}), 5.32(\mathrm{~s}, 2 \mathrm{H}), 5.39-5.74(\mathrm{~m}, 4 \mathrm{H}), 7.04-7.16(\mathrm{~m}, 2 \mathrm{H}), 7.18-7.32(\mathrm{~m}, 3 \mathrm{H}), 7.33(\mathrm{~s}, 1 \mathrm{H}), 7.63(\mathrm{~s}, 1 \mathrm{H})$; ${ }^{13} \mathrm{C} \mathrm{NMR}$ (100 MHz, $\mathrm{CDCl}_{3}$-TMS) $\delta$, ppm: 13.8, 13.9, 18.7, 19.6, 22.7, 26.4, 27.1, 27.6, 27.9, 36.8, 39.5, 44.7, 47.5, 49.0, 49.3, 49.4, 56.3, 58.8, 77.4, 88.0, 99.4, 120.9, 124.3, 127.7, 128.9, 129.4, 134.5, 141.7, 148.3, 159.8, 167.2, 170.2; IR (neat) $v, \mathrm{~cm}^{-1}: 3361,3136,3060,3027,2955,2927,2873,1754,1651,1551,1520,1497,1456,1324,1180,1131$, 958, 748, 703, 647; ESI-MS m/z (\%): 726 ([M+H $\left.]^{+}, 100\right), 748\left([\mathrm{M}+\mathrm{Na}]^{+}, 85\right)$; Anal. Calcd for $\mathrm{C}_{35} \mathrm{H}_{46} \mathrm{BrN}_{7} \mathrm{O}_{5}$ : C 58.01, H 6.40, N 13.53, Found: C 57.99, H 6.37, N 13.57.

(1-(4-(4-propyl-1H-1,2,3-triazol-1-yl)butyl)-1H-1,2,3-triazol-4-yl)methyl 6-((S)-4-bromo-5-oxo-2-((1S,2R,4S)-1,7,7-trimethylbicyclo[2.2.1]heptan-2-yloxy)-2,5-dihydrofuran-3-ylamino)hexanoate (5bg). Yellowish oil, yield $456 \mathrm{mg}(66 \%)$; $[\alpha]_{\mathrm{D}}{ }^{20}+117.6\left(c \quad 0.034, \mathrm{CH}_{2} \mathrm{Cl}_{2}\right) ; \mathrm{UV}_{-} \mathrm{vis}_{(\mathrm{C}}\left(\mathrm{CH}_{2} \mathrm{Cl}_{2}\right) \lambda_{\max }: 274 \mathrm{~nm}$; ${ }^{1} \mathrm{H} \mathrm{NMR}\left(400 \mathrm{MHz}, \mathrm{CDCl}_{3}\right.$ TMS) $\delta$, ppm: $0.86(\mathrm{~s}, 6 \mathrm{H}), 0.88(\mathrm{~s}, 3 \mathrm{H}), 0.97(\mathrm{t}, J=8.0 \mathrm{~Hz}, 3 \mathrm{H}), 1.22-1.31(\mathrm{~m}, 4 \mathrm{H}), 1.35-1.44(\mathrm{~m}, 2 \mathrm{H}), 1.65-1.87(\mathrm{~m}, 6 \mathrm{H}), 1.90-2.03(\mathrm{~m}, 4 \mathrm{H}), 2.21-2.29(\mathrm{~m}, 1 \mathrm{H}), 2.36(\mathrm{t}, J=$ $8.0 \mathrm{~Hz}, 2 \mathrm{H}), 2.68(\mathrm{t}, J=8.0 \mathrm{~Hz}, 2 \mathrm{H}), 3.38-3.57(\mathrm{~m}, 2 \mathrm{H}), 3.99(\mathrm{~d}, J=12.0 \mathrm{~Hz}, 1 \mathrm{H}), 4.29-4.46(\mathrm{~m}, 4 \mathrm{H}), 5.20(\mathrm{~s}, 2 \mathrm{H}), 5.41(\mathrm{~s}, 1 \mathrm{H}), 5.77(\mathrm{~s}, 1 \mathrm{H}), 7.32(\mathrm{~s}, 1 \mathrm{H}), 7.62(\mathrm{~s}, 1 \mathrm{H}) ;{ }^{13} \mathrm{C}$ NMR (100 MHz, $\mathrm{CDCl}_{3}$-TMS) $\delta$, ppm: 13.8, 14.0, 18.8, 19.6, 22.7, 24.2, 25.8, 26.6, 27.1, 27.6, 28.0, 30.2, 33.7, 37.1, 43.9, 44.8, 47.6, 49.1, 49.4, 57.5, 77.3, 87.4, 99.1, 120.8, 123.7, 142.8, 148.4, 160.3, 167.8, 173.2; IR (neat) $v, \mathrm{~cm}^{-1}: 3313,3136,3084,2954,2931,2873,1744,1650,1552,1528,1458,1321,1156,1132,955,634$; ESI-MS m/z (\%): $692\left([\mathrm{M}+\mathrm{H}]^{+}, 81\right), 714\left([\mathrm{M}+\mathrm{Na}]^{+}, 100\right)$; Anal. Calcd for $\mathrm{C}_{32} \mathrm{H}_{48} \mathrm{BrN}_{7} \mathrm{O}_{5}$ : C 55.65, H 7.00, N 14.20, Found: C 55.70, H 7.03, N 14.18.

(1-(4-(4-propyl-1H-1,2,3-triazol-1-yl)butyl)-1H-1,2,3-triazol-4-yl)methyl 4-((S)-4-bromo-5-oxo-2-((1S,2R,4S)-1,7,7-trimethylbicyclo[2.2.1]heptan-2-yloxy)-2,5-dihydrofuran-3-ylamino)butanoate (5bh). Yellowish solid, yield $652 \mathrm{mg}(96 \%)$; m.p. 94.7-95.6 ${ }^{\circ} \mathrm{C}$.; $[\alpha]_{\mathrm{D}}^{20}+51.8\left(c{ }^{0.073}, \mathrm{CH}_{2} \mathrm{Cl}_{2}\right)$; $\mathrm{UV}^{2}$-vis $\left(\mathrm{CH}_{2} \mathrm{Cl}_{2}\right) \lambda_{\text {max }}$ : $272 \mathrm{~nm}$; ${ }^{1} \mathrm{H} \mathrm{NMR}$ $\left(400 \mathrm{MHz}, \mathrm{CDCl}_{3}-\mathrm{TMS}\right) \delta$, ppm: 0.72-0.93 (m, 9H), $0.96(\mathrm{t}, J=8.0 \mathrm{~Hz}, 3 \mathrm{H}), 1.13-1.37(\mathrm{~m}, 4 \mathrm{H}), 1.64-1.87(\mathrm{~m}, 4 \mathrm{H}), 1.88-2.06(\mathrm{~m}, 6 \mathrm{H}), 2.18-2.28(\mathrm{~m}, 1 \mathrm{H}), 2.48(\mathrm{t}, J=8.0 \mathrm{~Hz}$, $2 \mathrm{H}), 2.67(\mathrm{t}, J=8.0 \mathrm{~Hz}, 2 \mathrm{H}), 3.45-3.66(\mathrm{~m}, 2 \mathrm{H}), 3.99(\mathrm{~d}, J=8.0 \mathrm{~Hz}, 1 \mathrm{H}), 4.30-4.47(\mathrm{~m}, 4 \mathrm{H}), 5.21(\mathrm{~s}, 2 \mathrm{H}), 5.80(\mathrm{~s}, 1 \mathrm{H}), 6.16(\mathrm{~s}, 1 \mathrm{H}), 7.38(\mathrm{~s}, 1 \mathrm{H}), 7.70(\mathrm{~s}, 1 \mathrm{H}) ;{ }^{13} \mathrm{C} \mathrm{NMR}(100$ 
$\mathrm{MHz}, \mathrm{CDCl}_{3}$-TMS) $\delta$, ppm: 13.8, 13.9, 18.7, 19.5, 22.6, 25.3, 26.5, 27.1, 27.1, 27.5, 27.9, 31.0, 37.0, 43.3, 44.7, 47.5, 49.1, 49.3, 49.4, 57.7, 77.5, 87.3, 99.1, 120.9, 123.8, 142.5, 148.2, 160.7, 167.9, 172.8; IR (neat) $v, \mathrm{~cm}^{-1}: 3318,3137,3081,2955,2931,2874,1745,1650,1551,1457,1322,1166,1131,953,661 ; \mathrm{ESI}-\mathrm{MS} m / z(\%): 686$ ([M+Na] $]^{+}$ 100); Anal. Calcd for $\mathrm{C}_{30} \mathrm{H}_{44} \mathrm{BrN}_{7} \mathrm{O}_{5}$ : C 54.38, H 6.69, N 14.80, Found: C 54.40, H 6.72, N 14.79. 


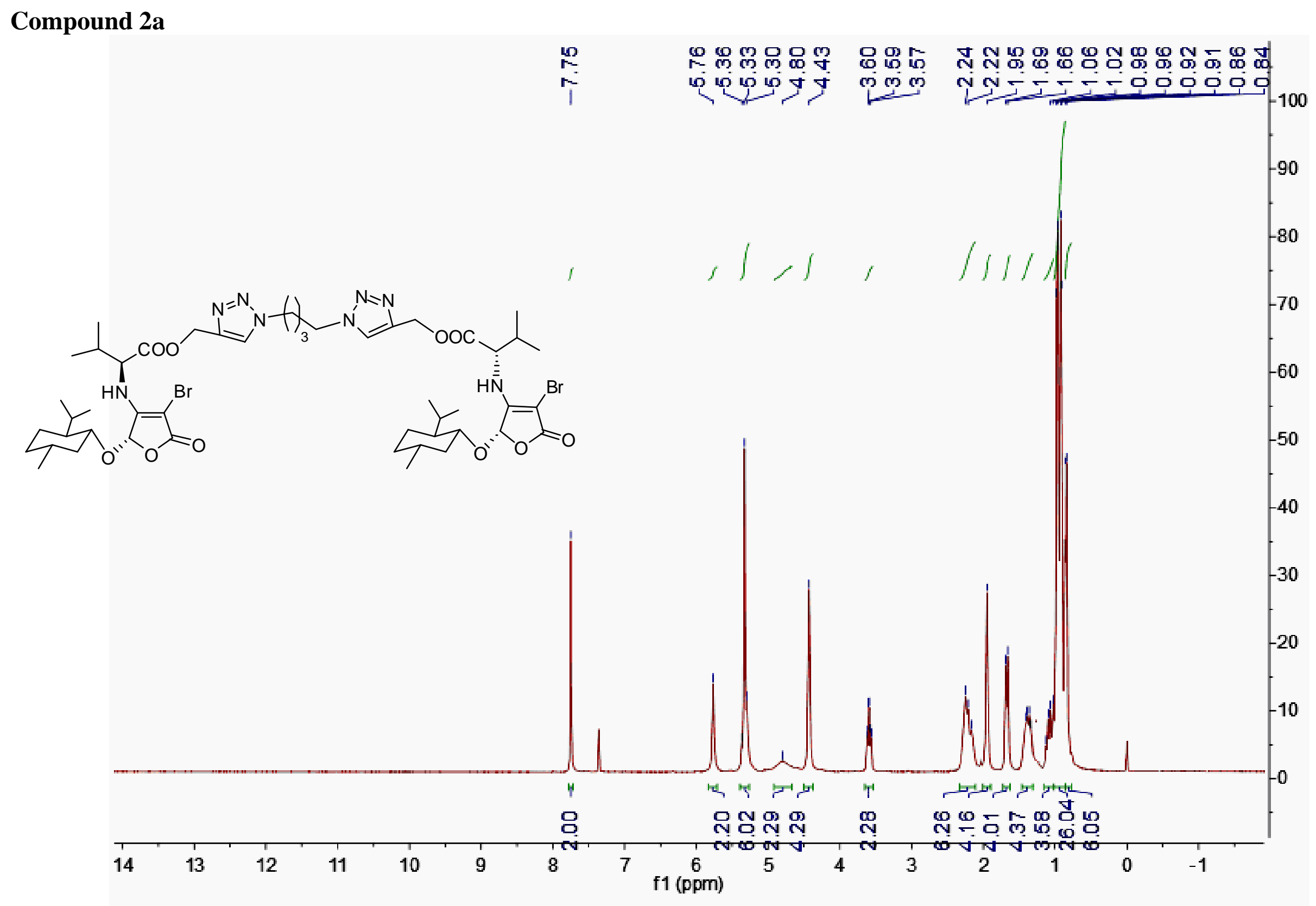




\section{Compound 2a}

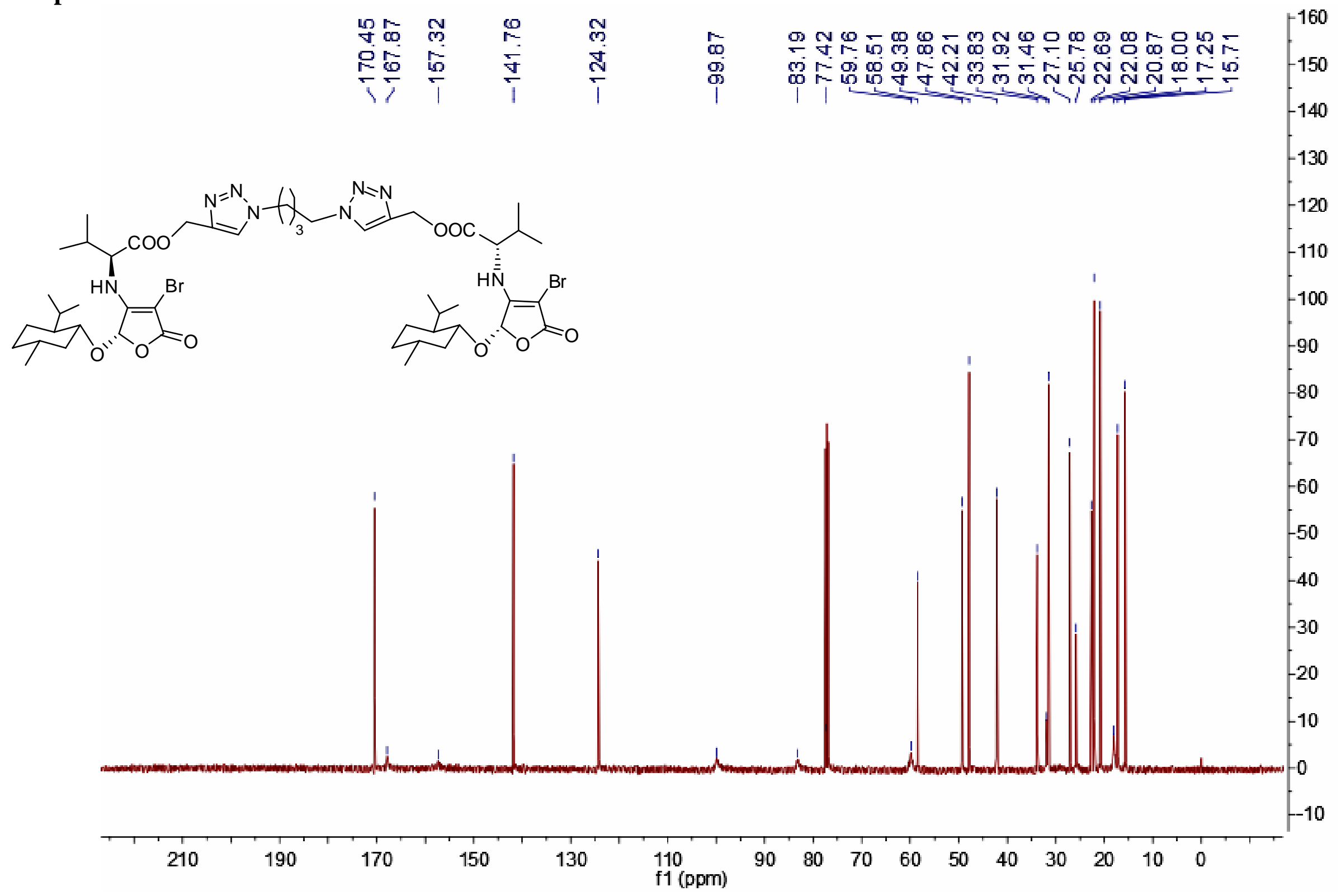




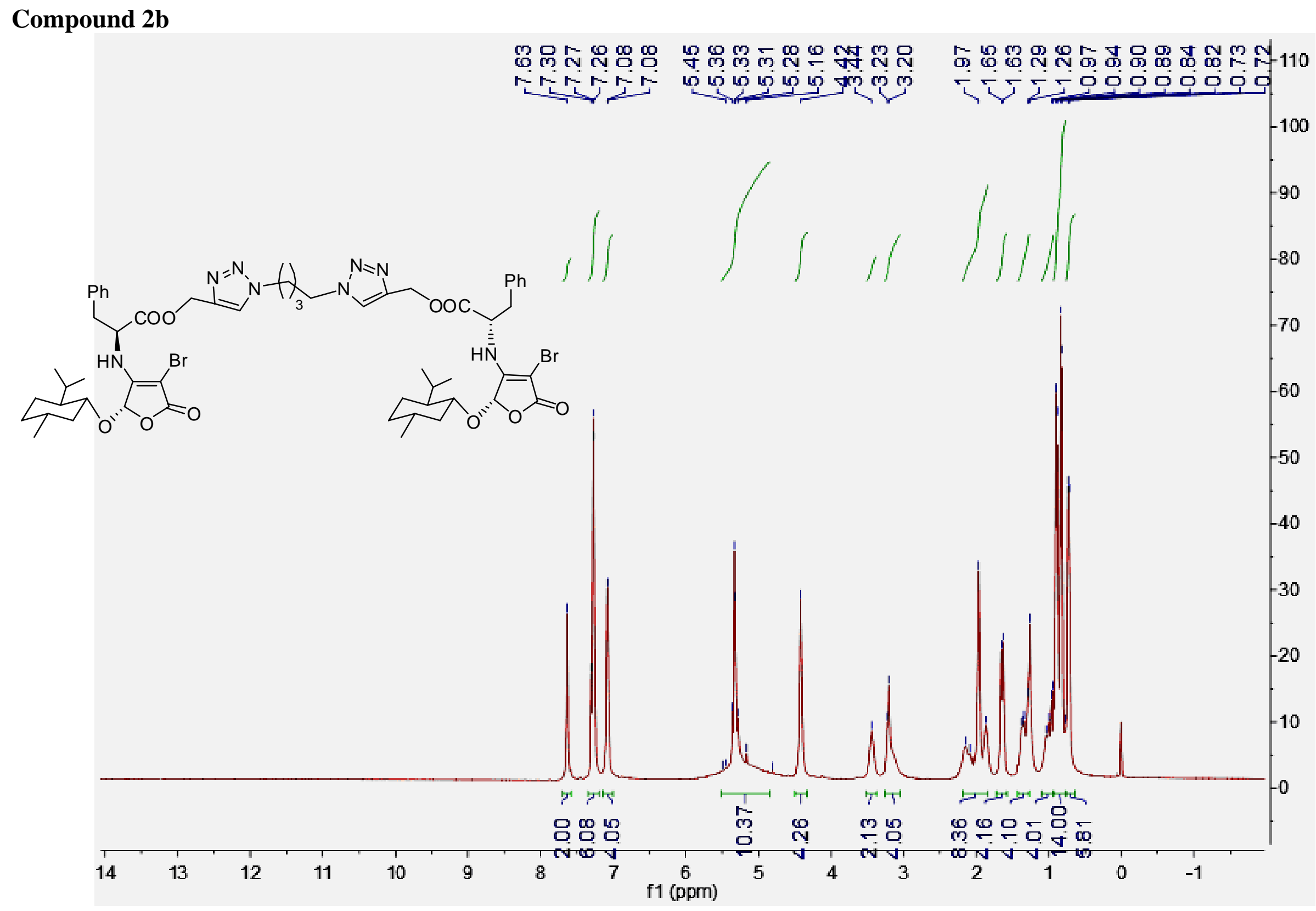




\section{Compound $2 b$}

กฺ

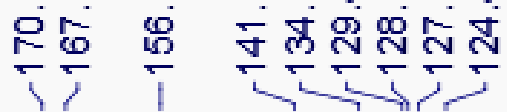

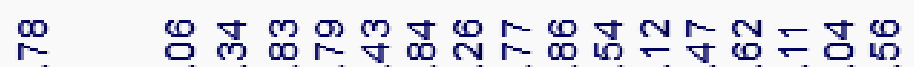

$-220$

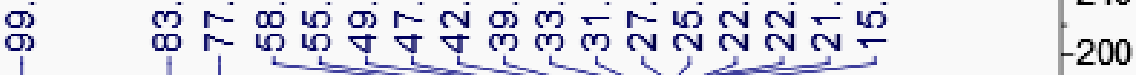

190

$-180$

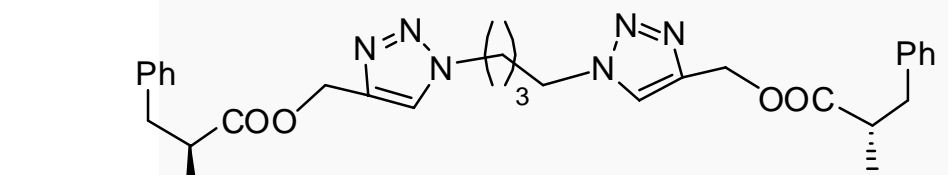

$Y_{0}=\sum_{0}^{B r}$
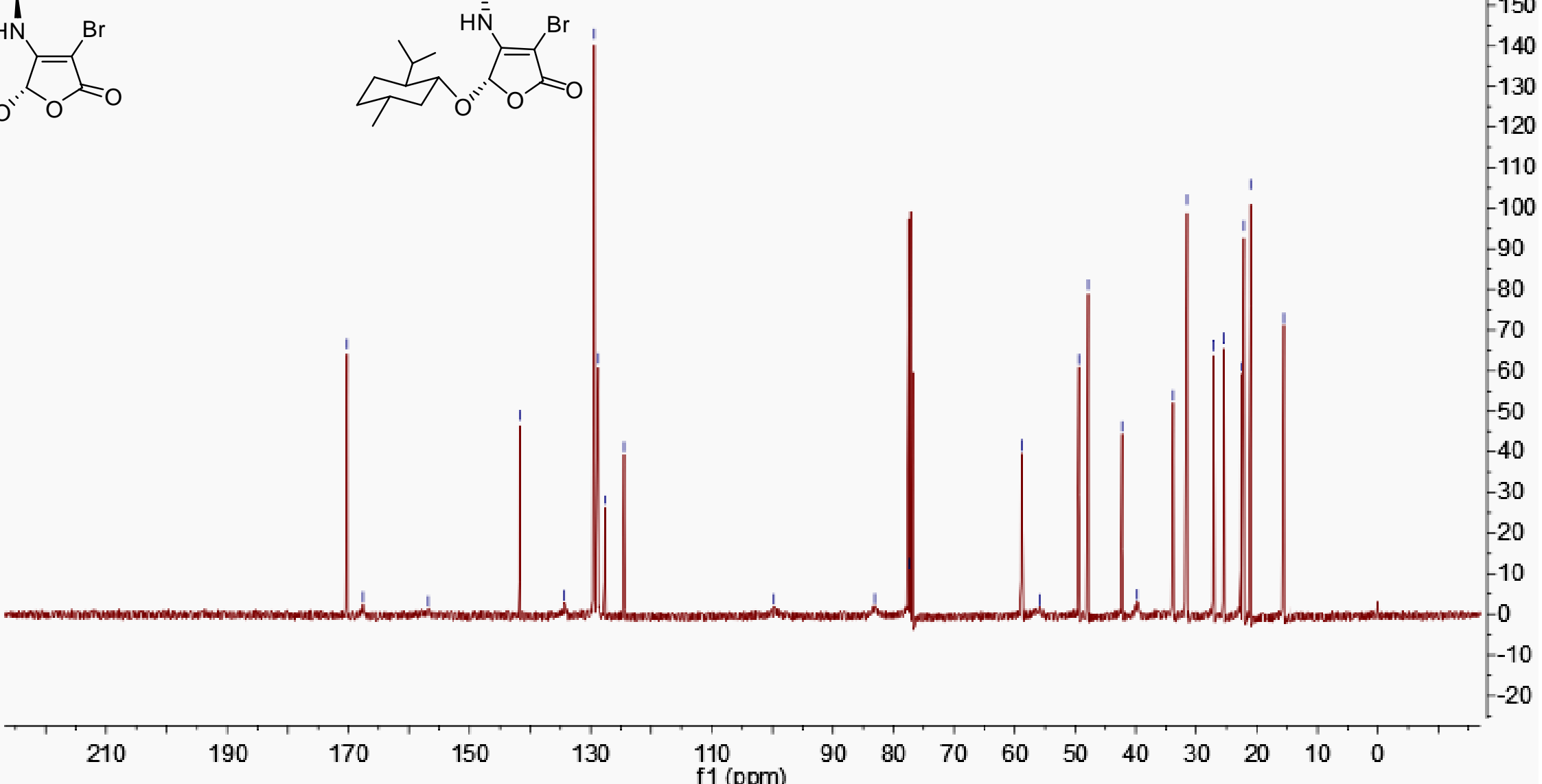


\section{Compound 2c}

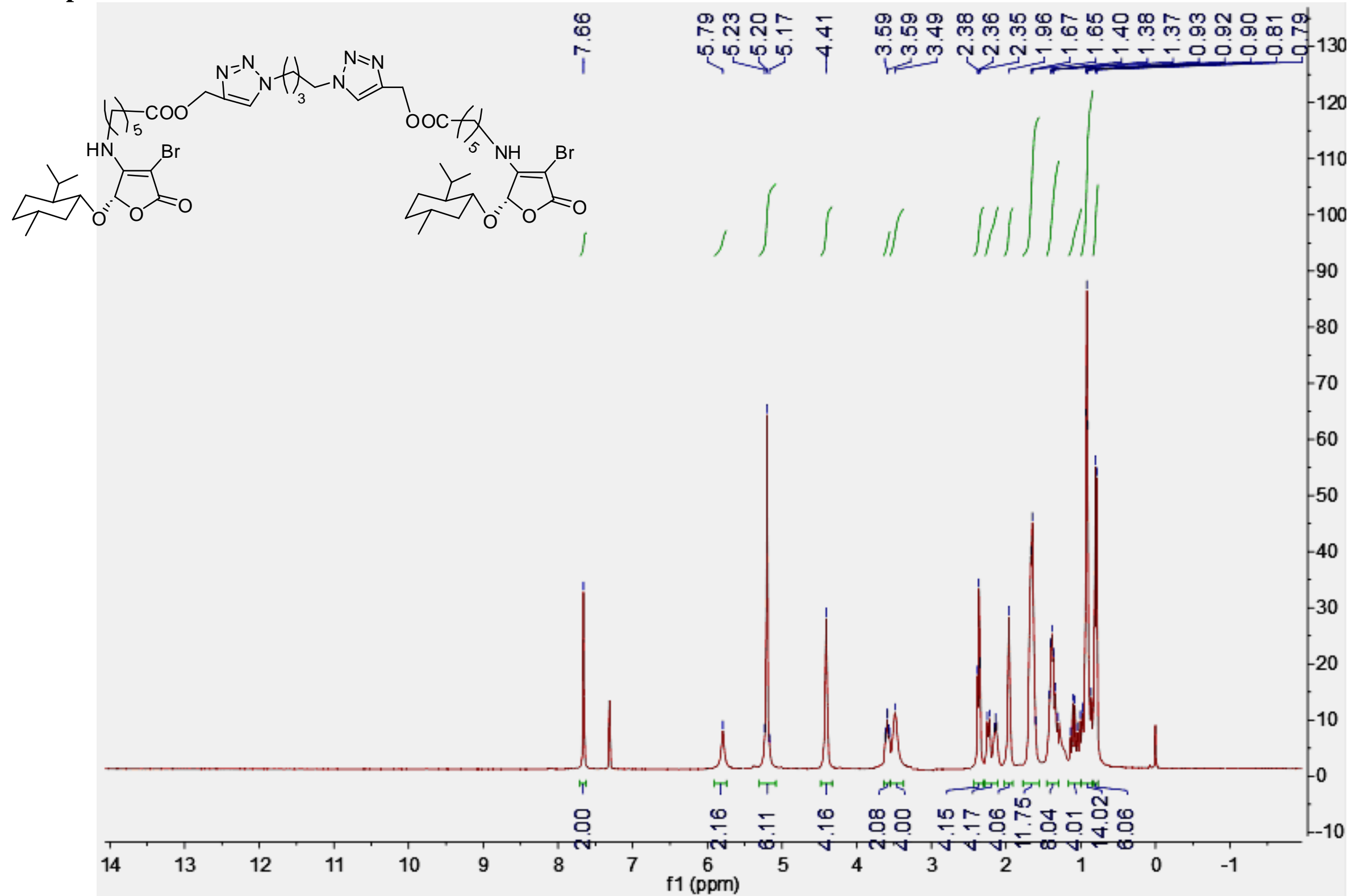




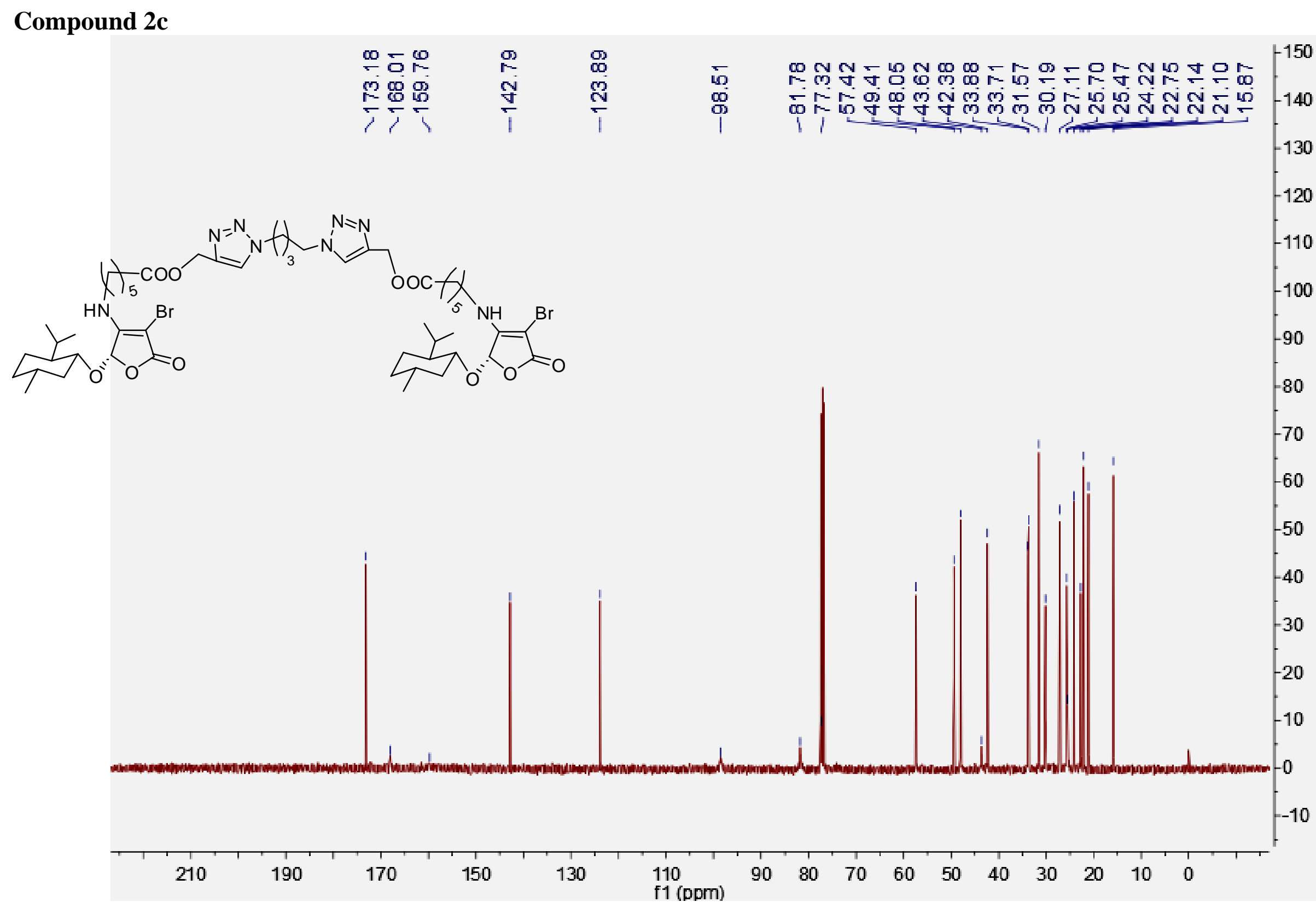




\section{Compound 2d}

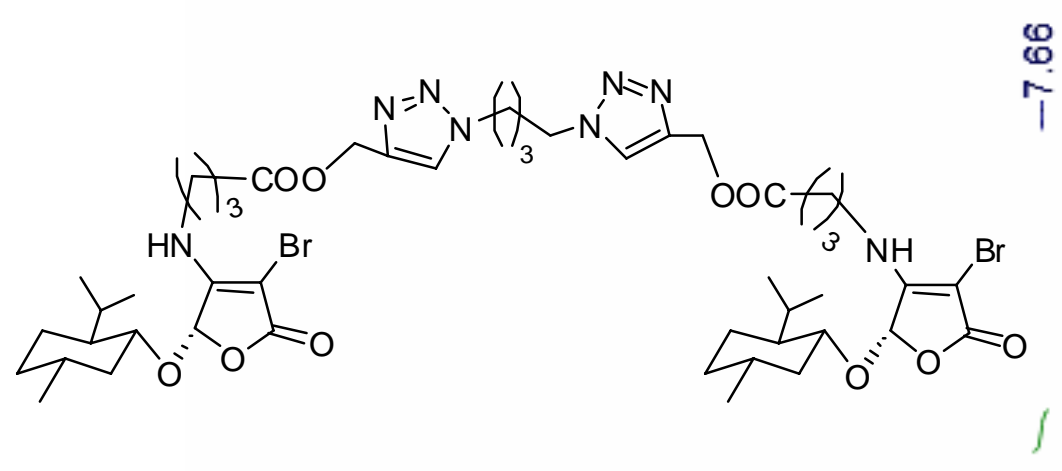




\section{Compound 2d}

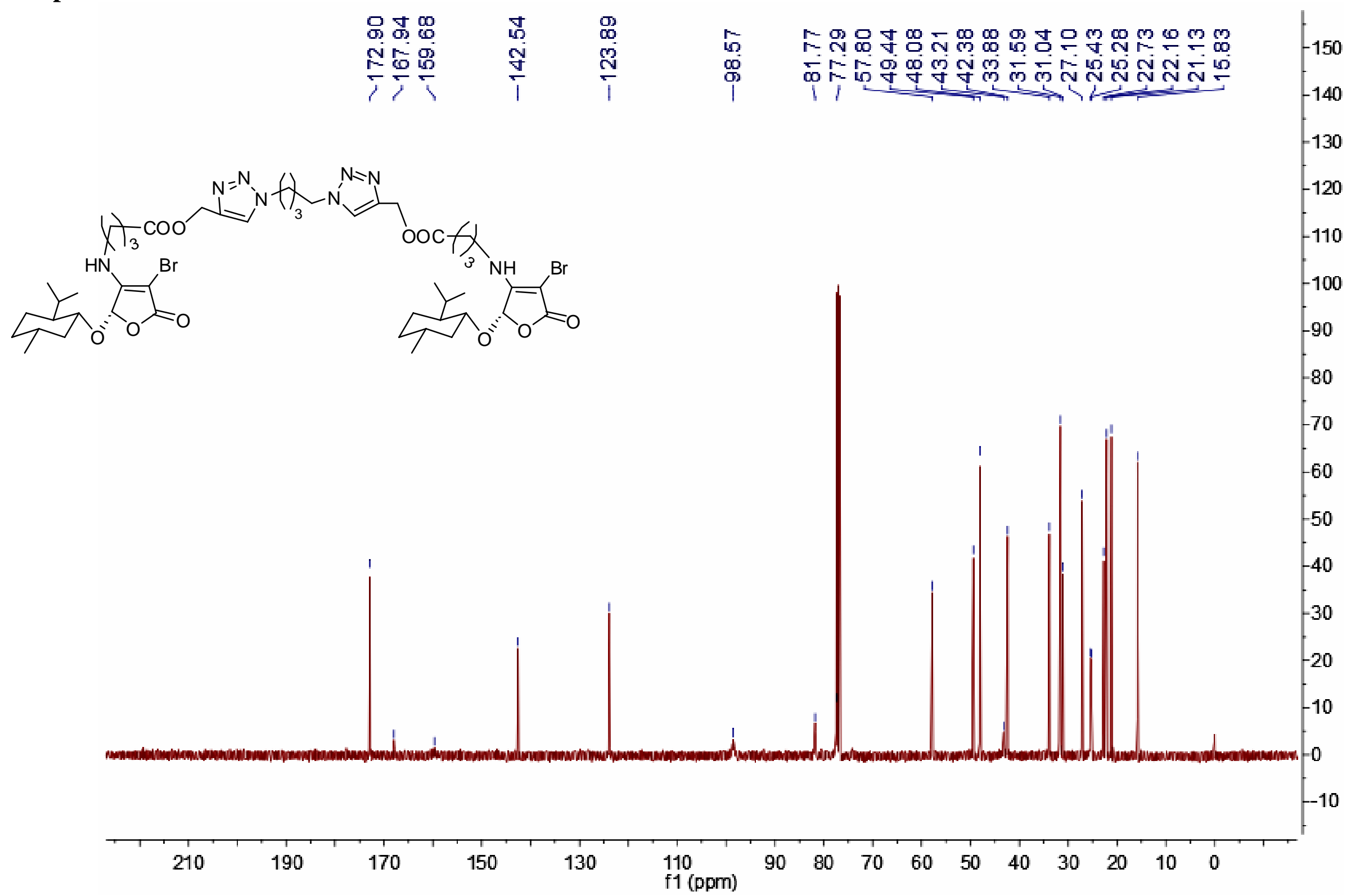




\section{Compound $2 \mathbf{e}$}

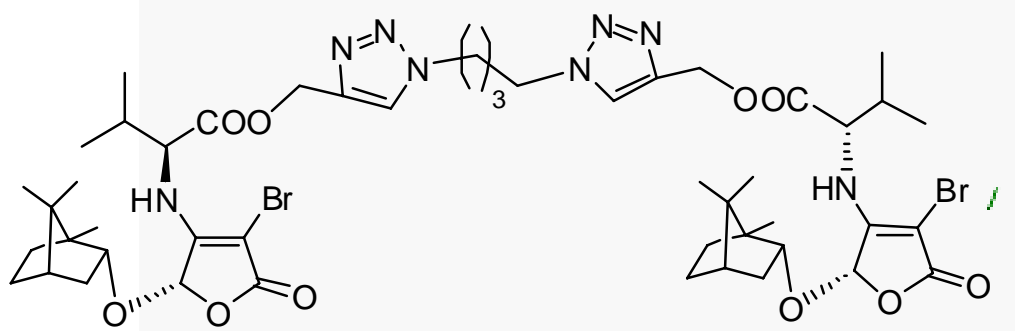

뜜ำ

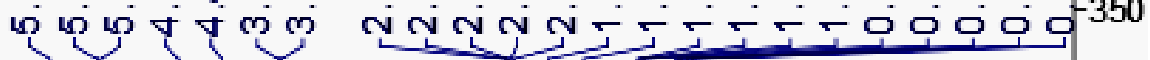

ri
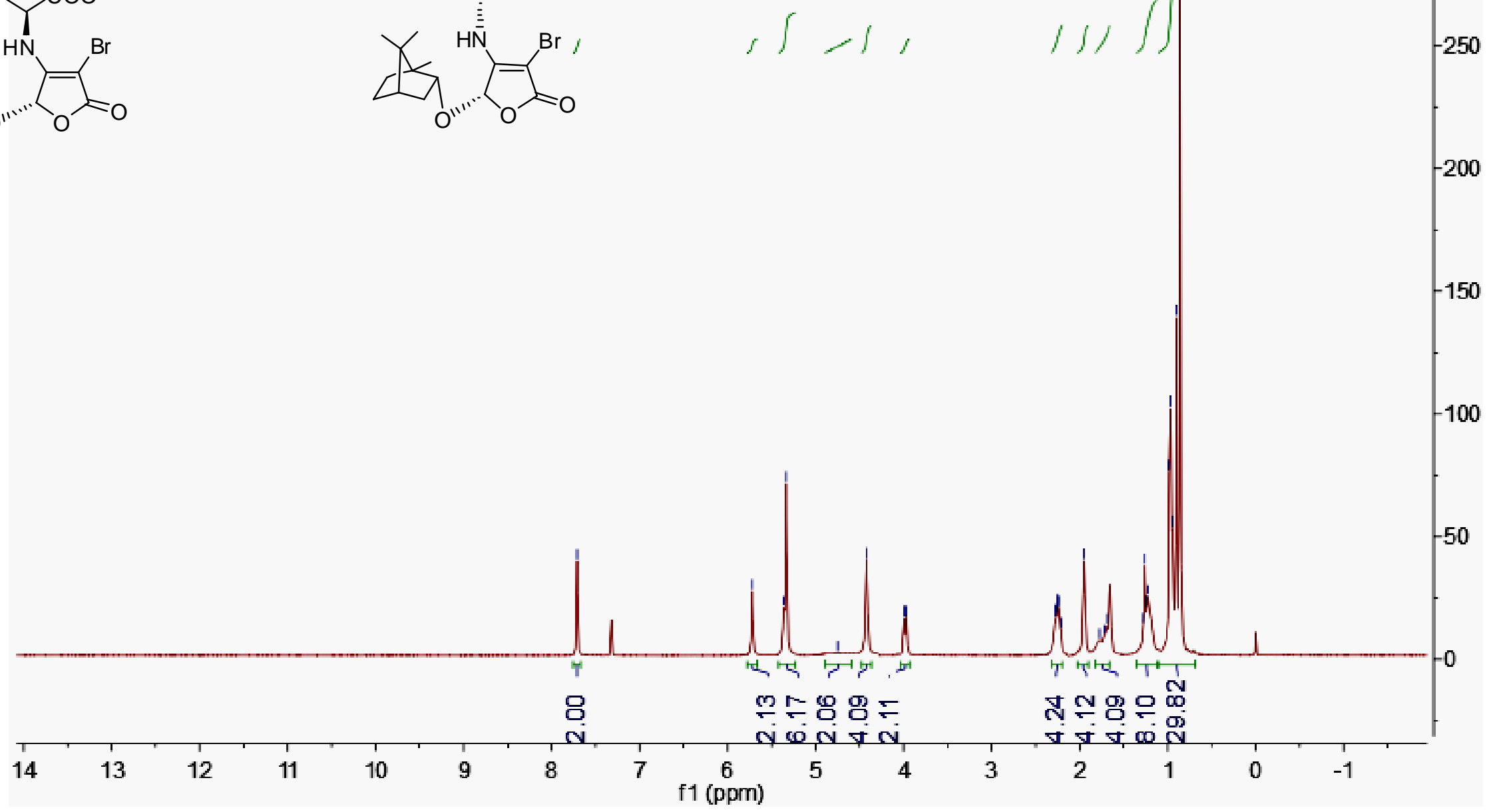


\section{Compound 2 e}

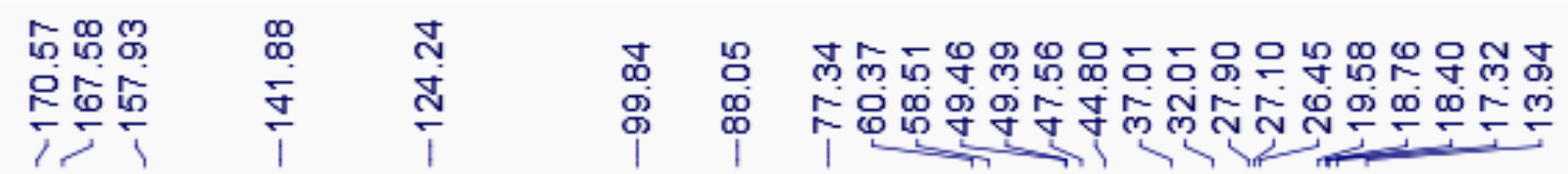
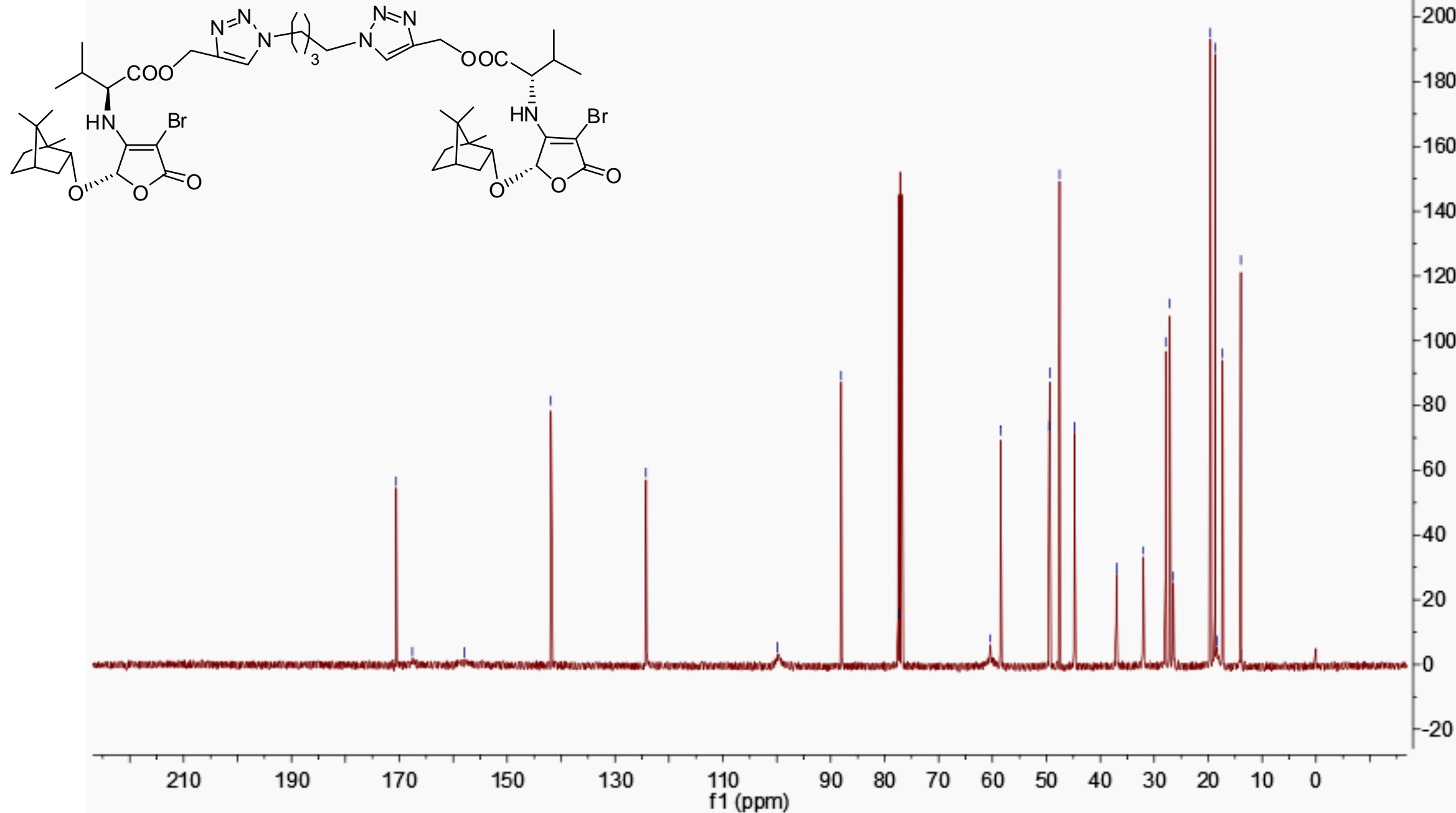


\section{Compound $2 f$}

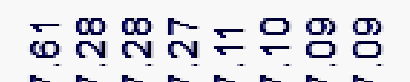

rmmmin

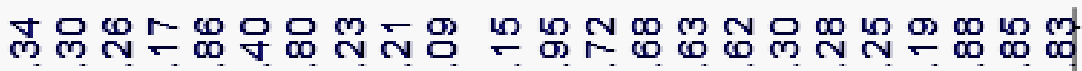

ט

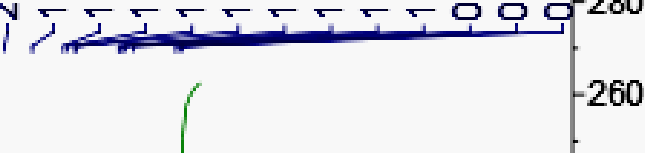<smiles>C=CC(=O)OCc1cn(CC(C)(C)n2cc(COC(=O)[C@H](Cc3ccccc3)NC3=C(Br)C(=O)O[C@@H]3C3CC4CCC3(C)C4(C)C)nn2)nn1</smiles>

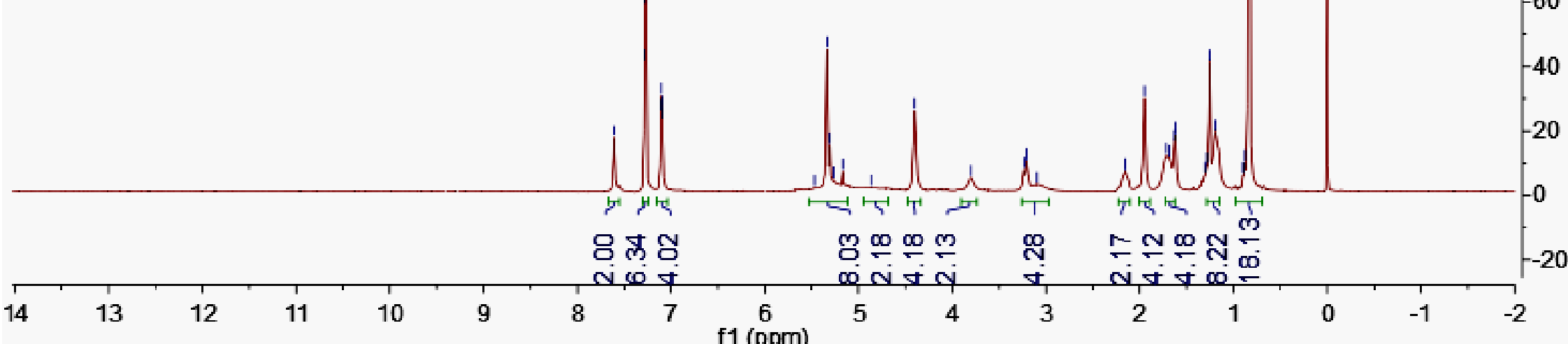




\section{Compound $2 f$}

m

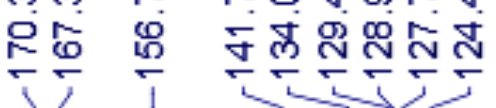

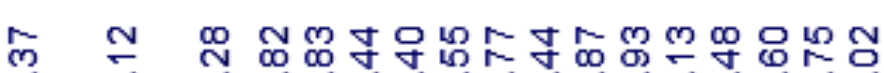

क

$-380$

$-360$
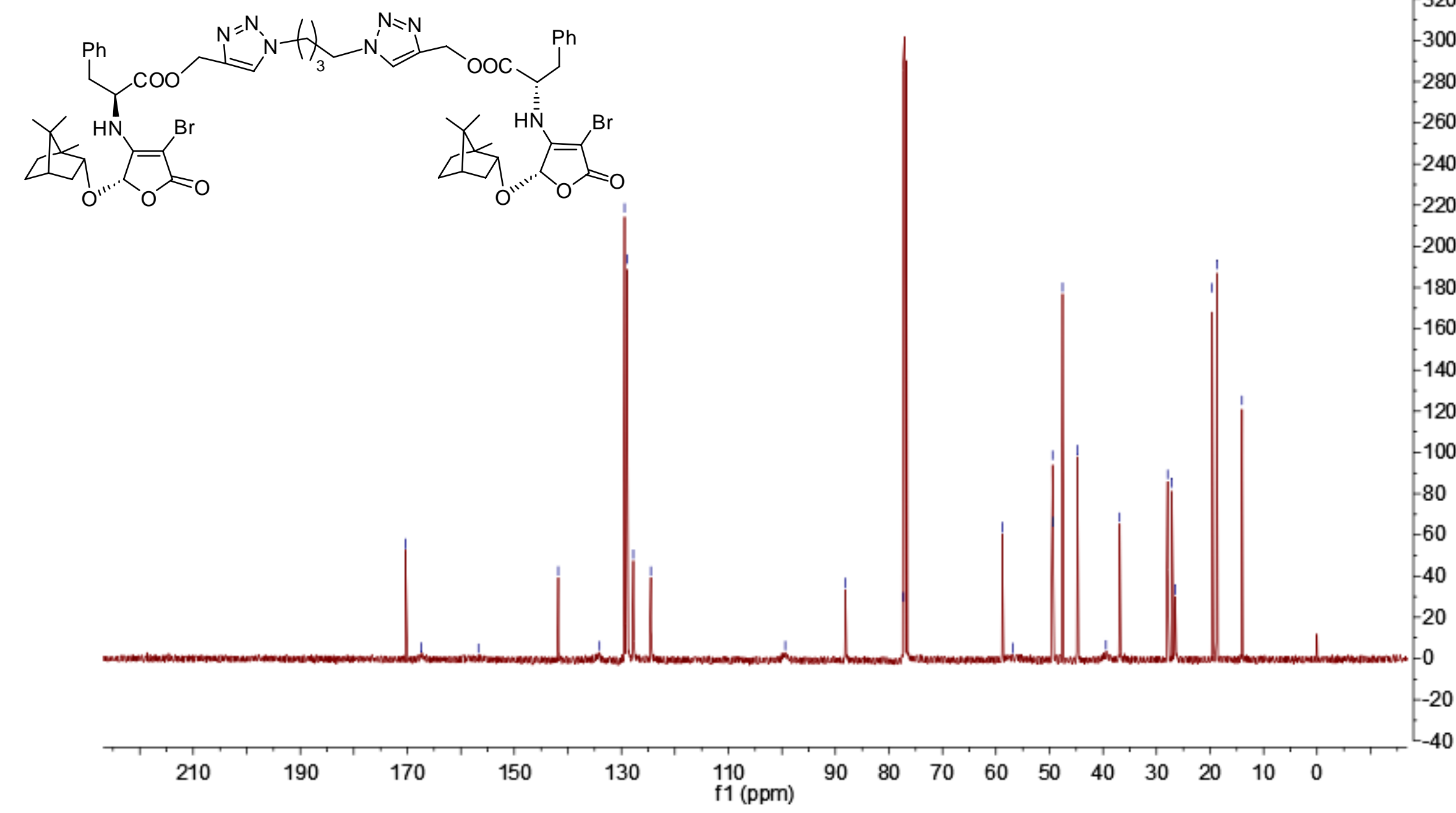


\section{Compound 2g}

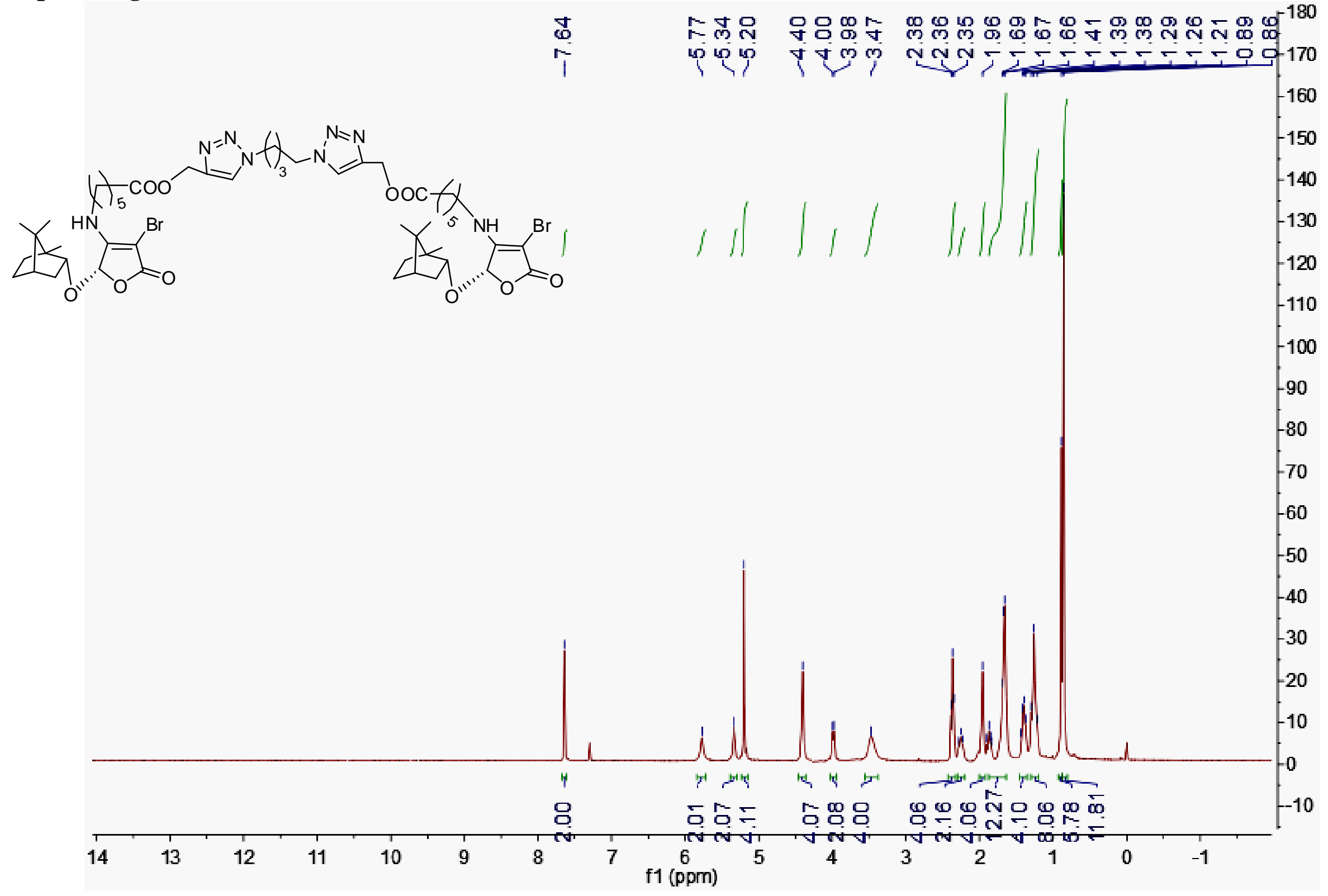




\section{Compound 2g}

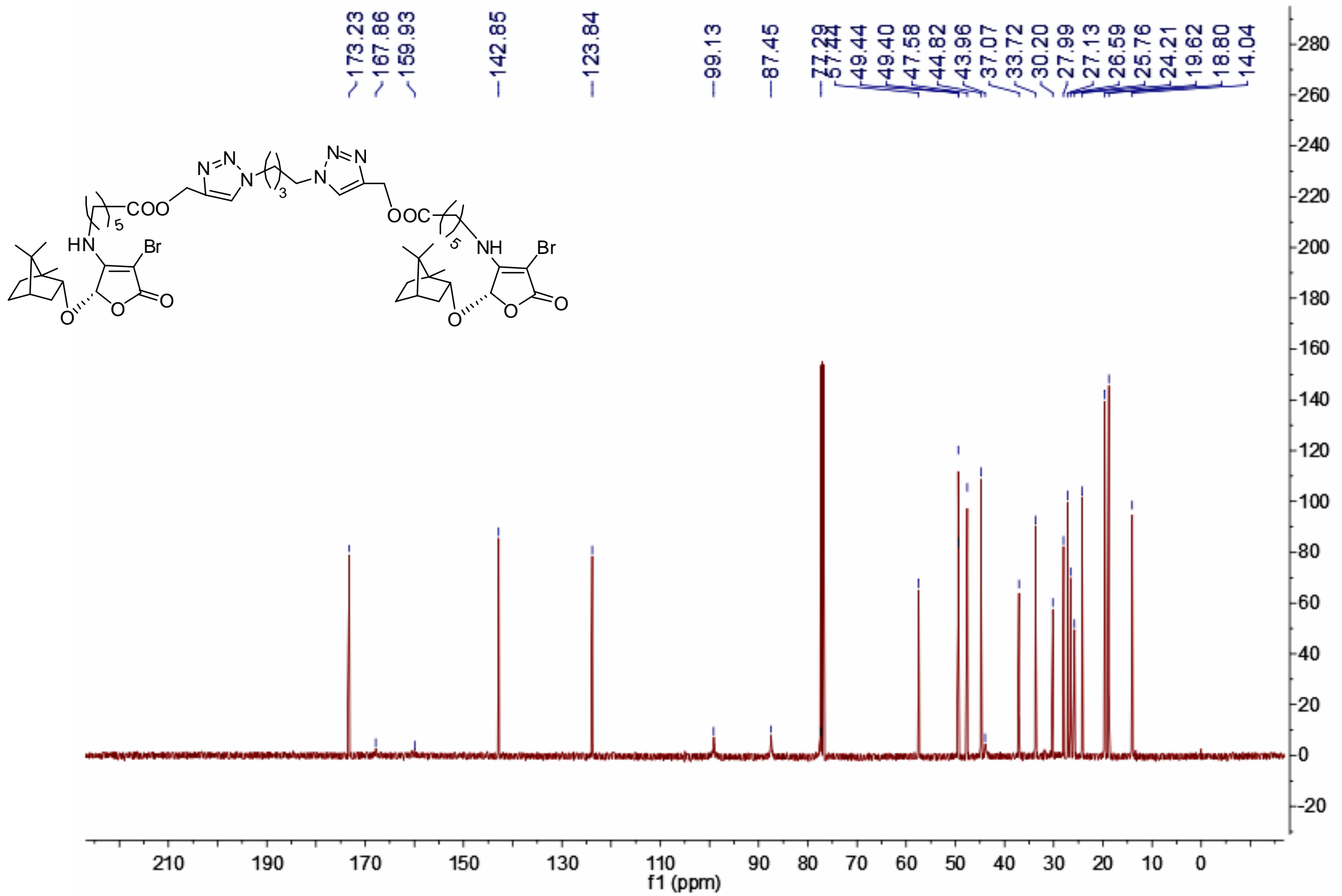




\section{Compound 2h}

ம

i ம
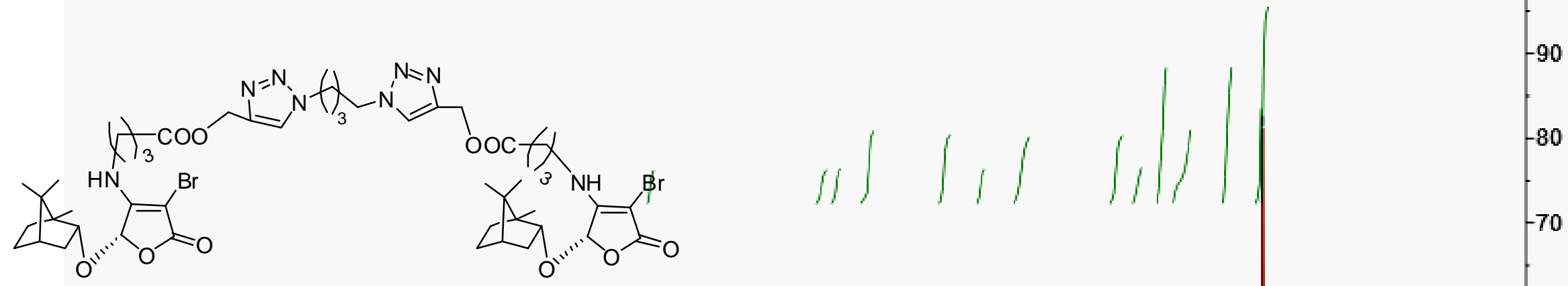

(") 0

O"“"
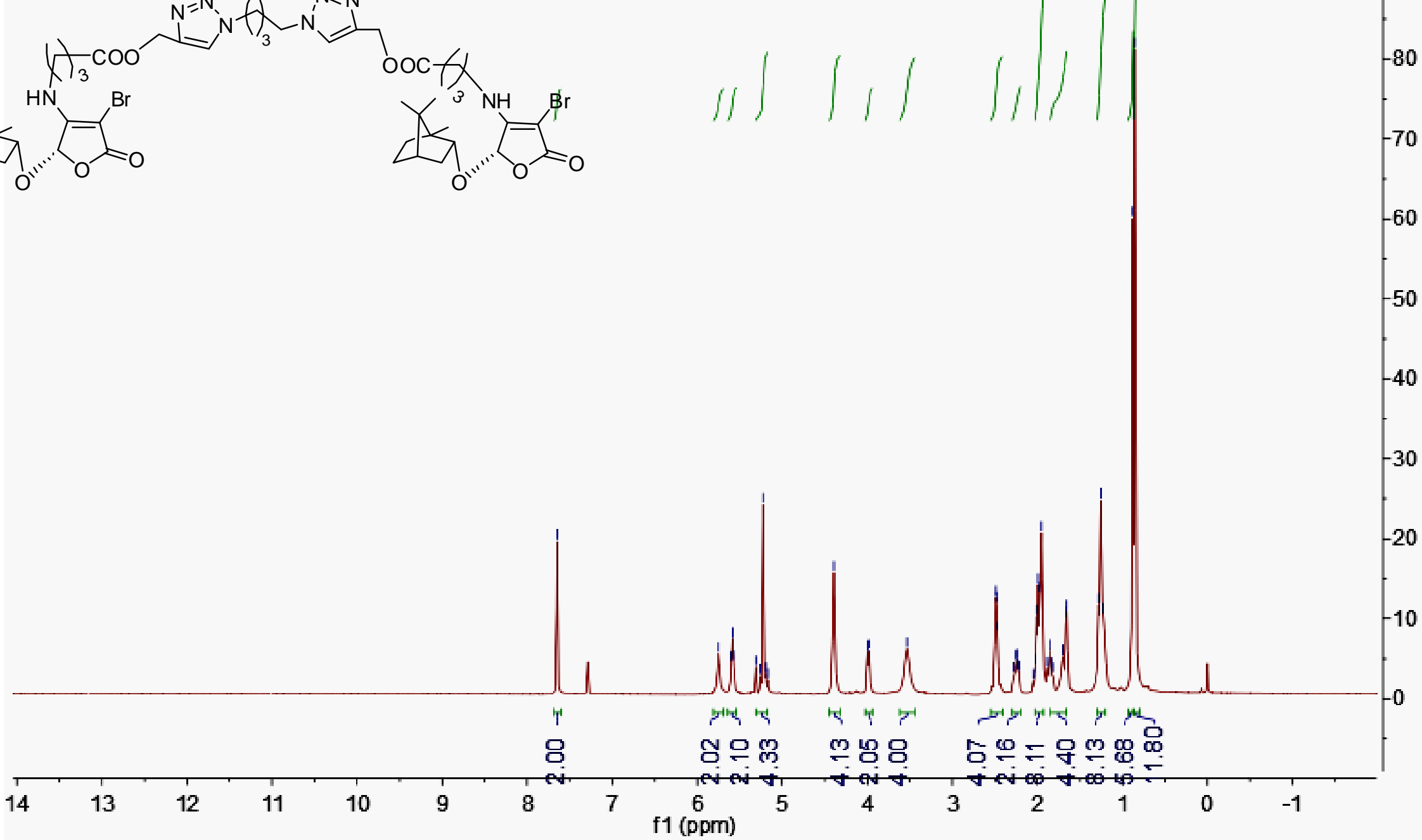


\section{Compound 2h}

$$
\begin{aligned}
& \text { 仕员 员 } \\
& \text { लํํㅇ }
\end{aligned}
$$

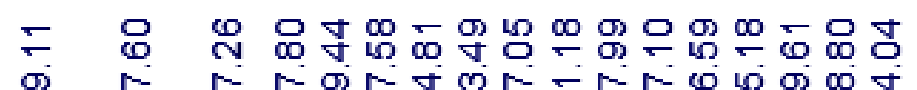

$$
\begin{aligned}
& \text { क }
\end{aligned}
$$
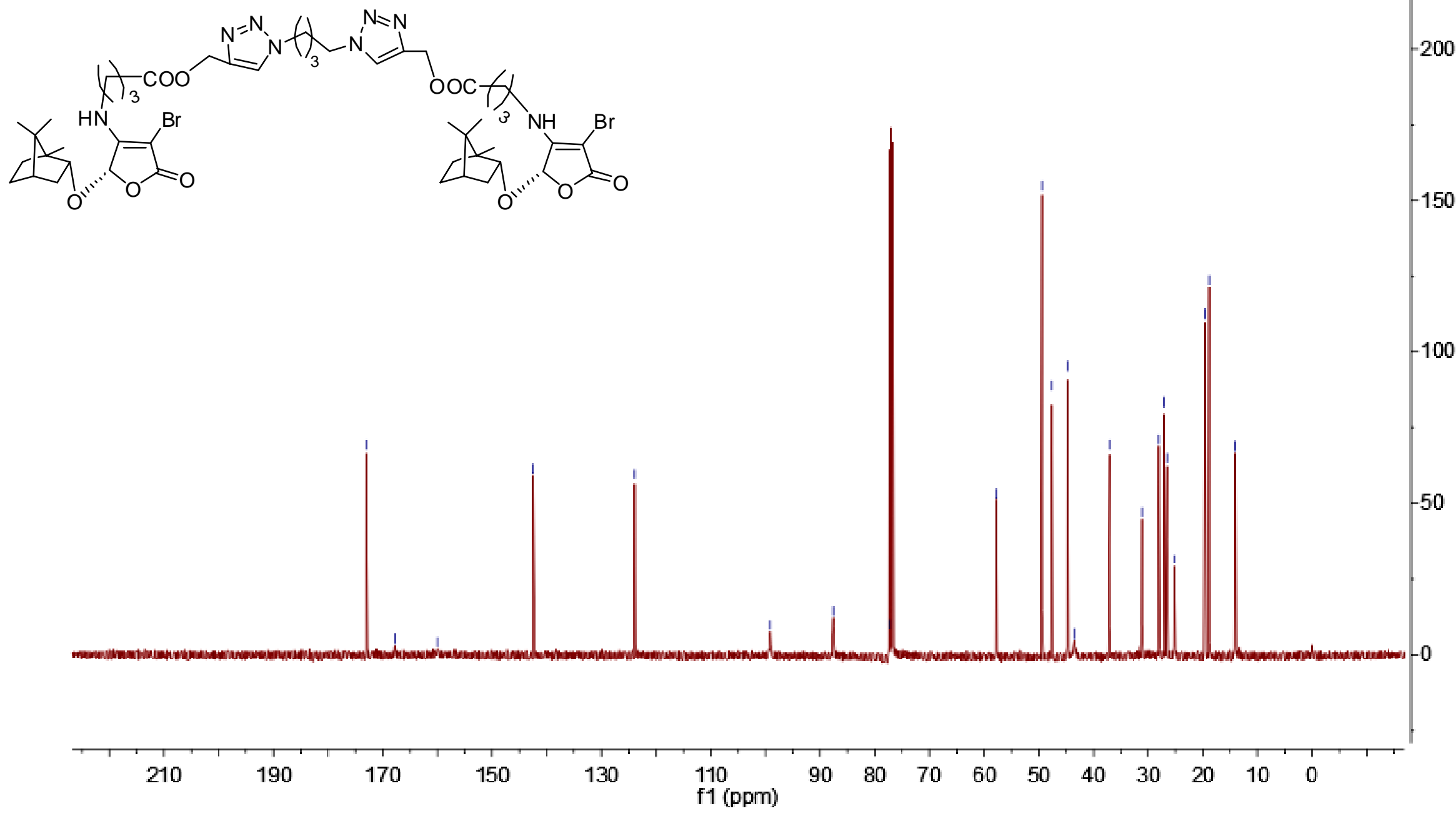


\section{Compound 3a}

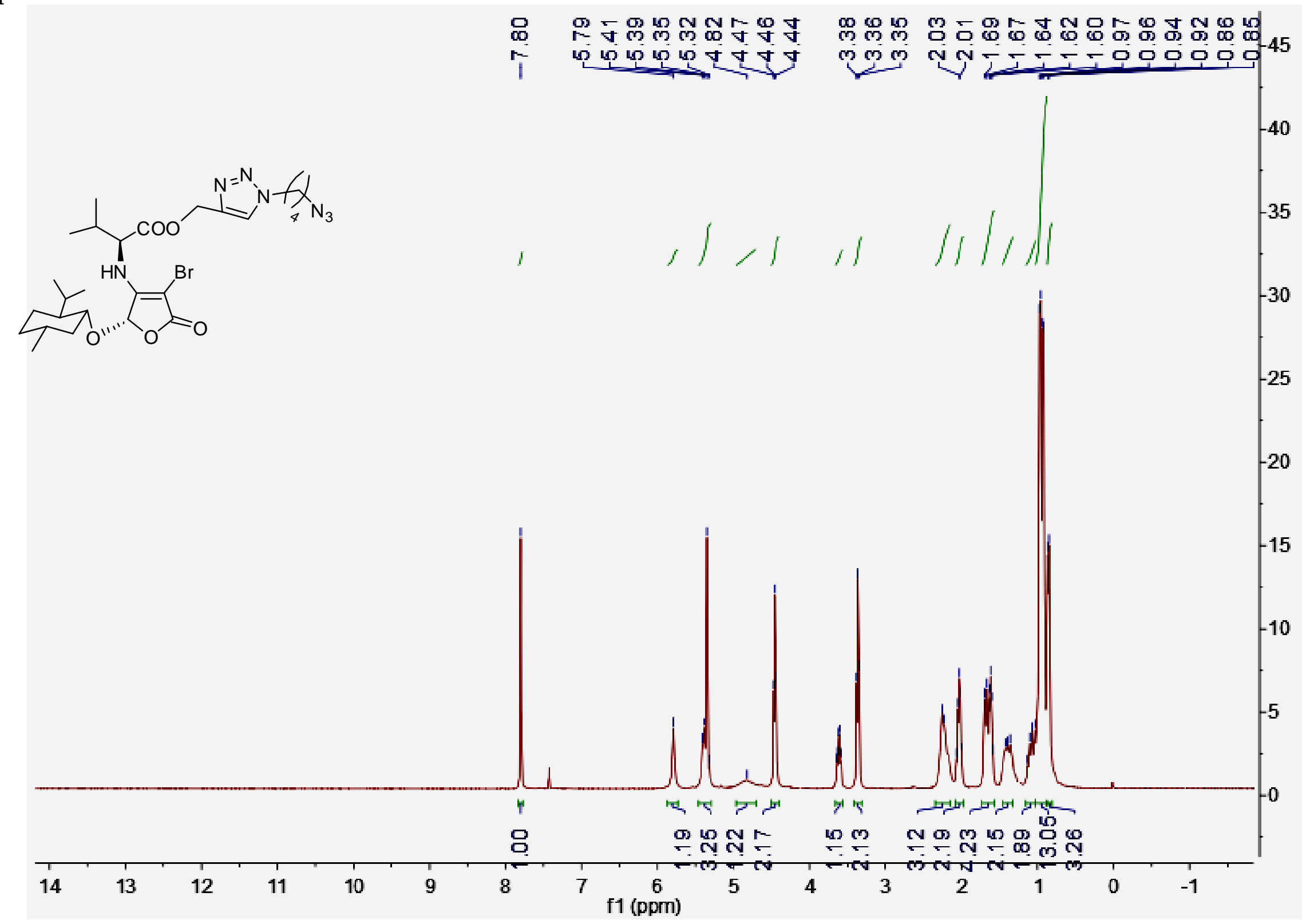




\section{Compound 3a}

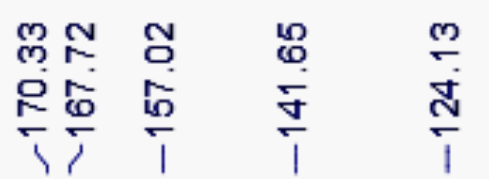

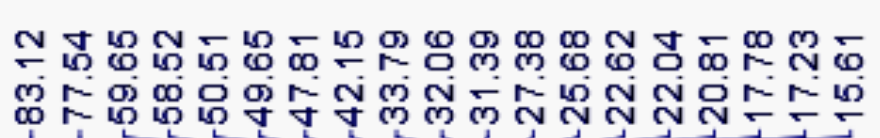

।
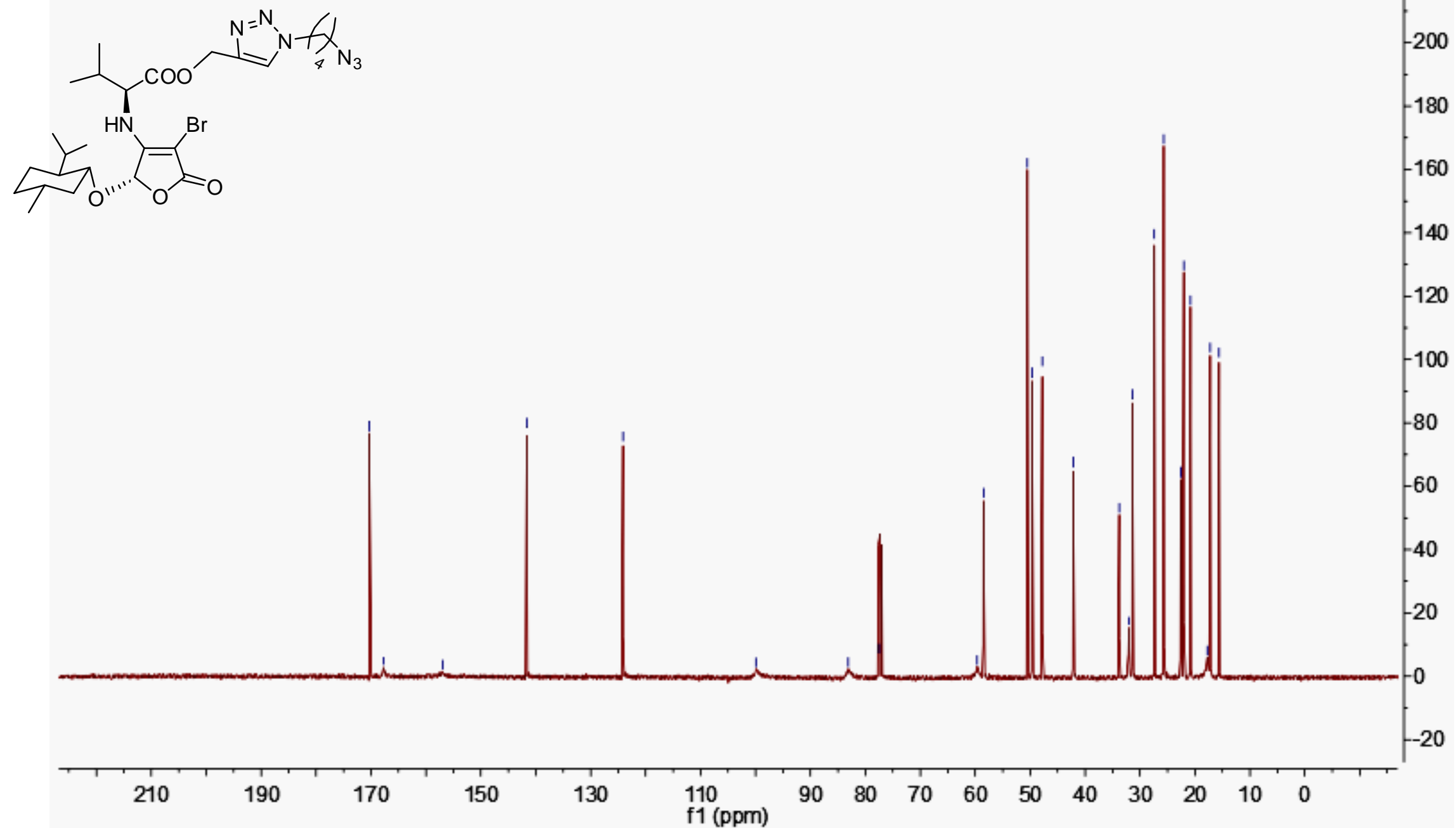


\section{Compound 3b}

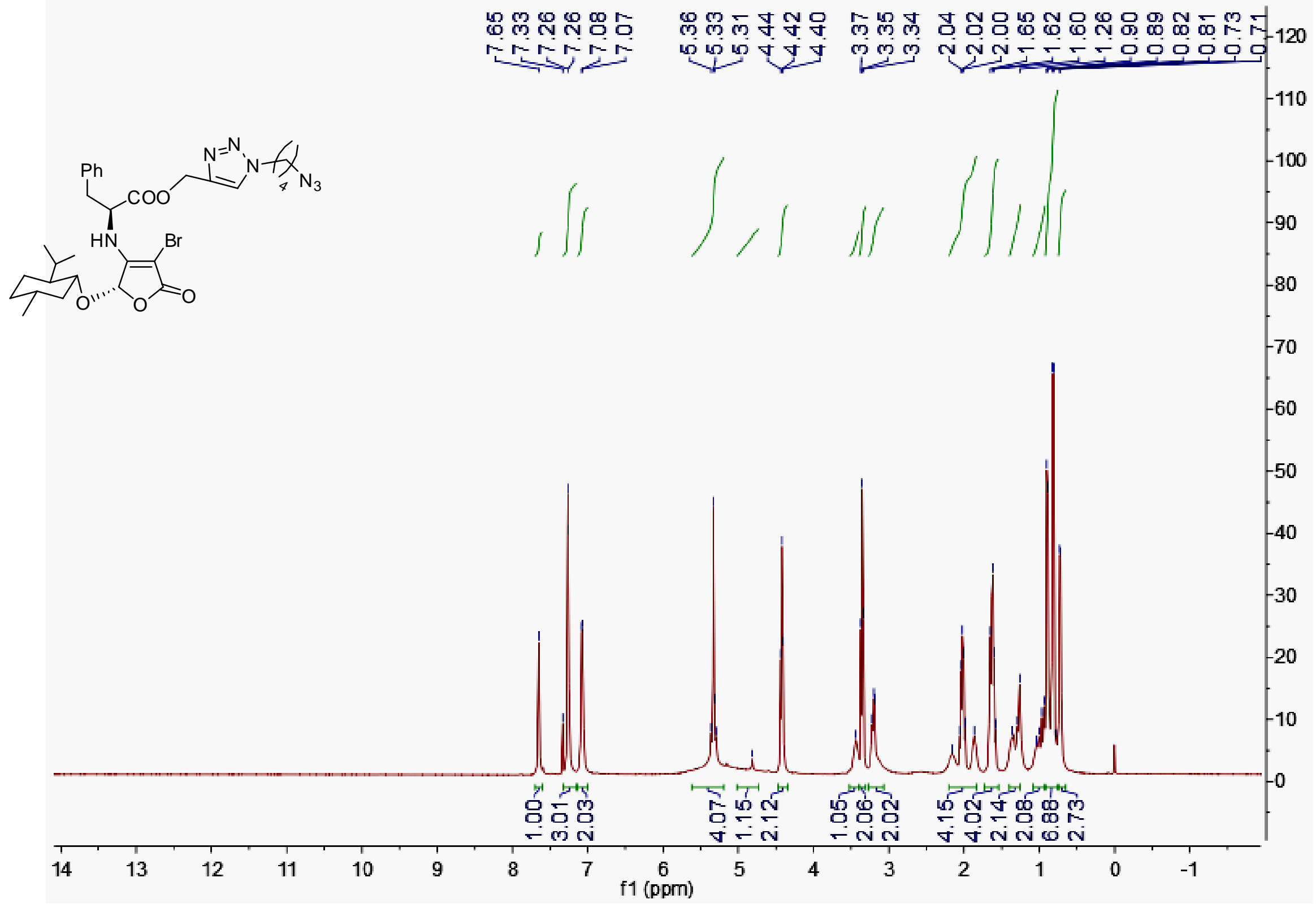




\section{Compound 3b}

ㅁ

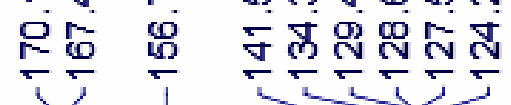

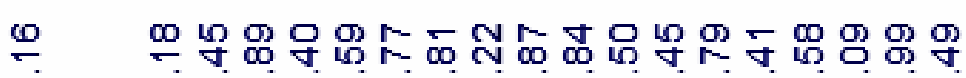
以
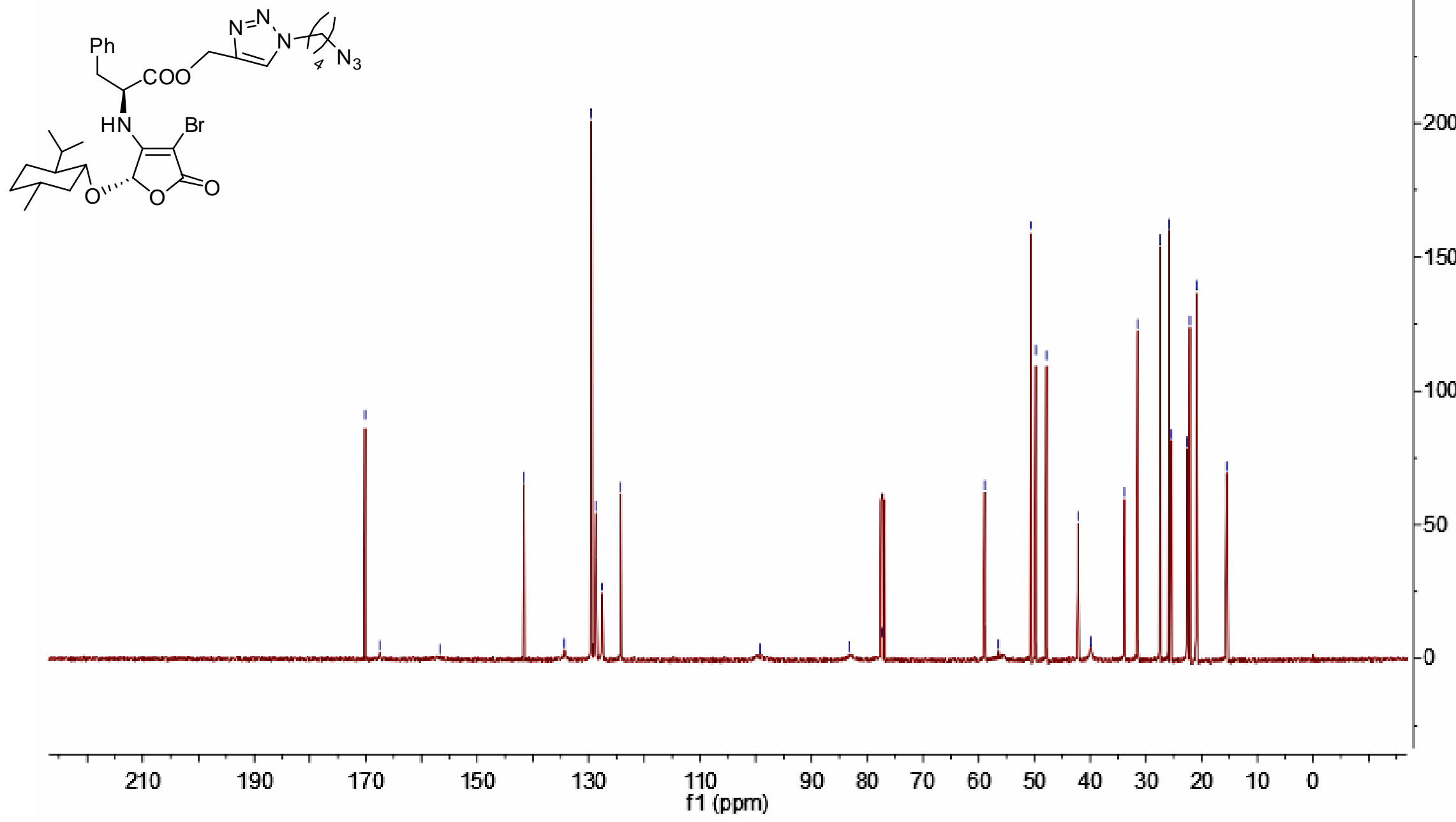


\section{Compound 3c}

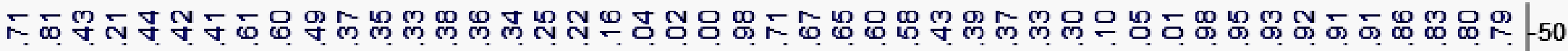

m n
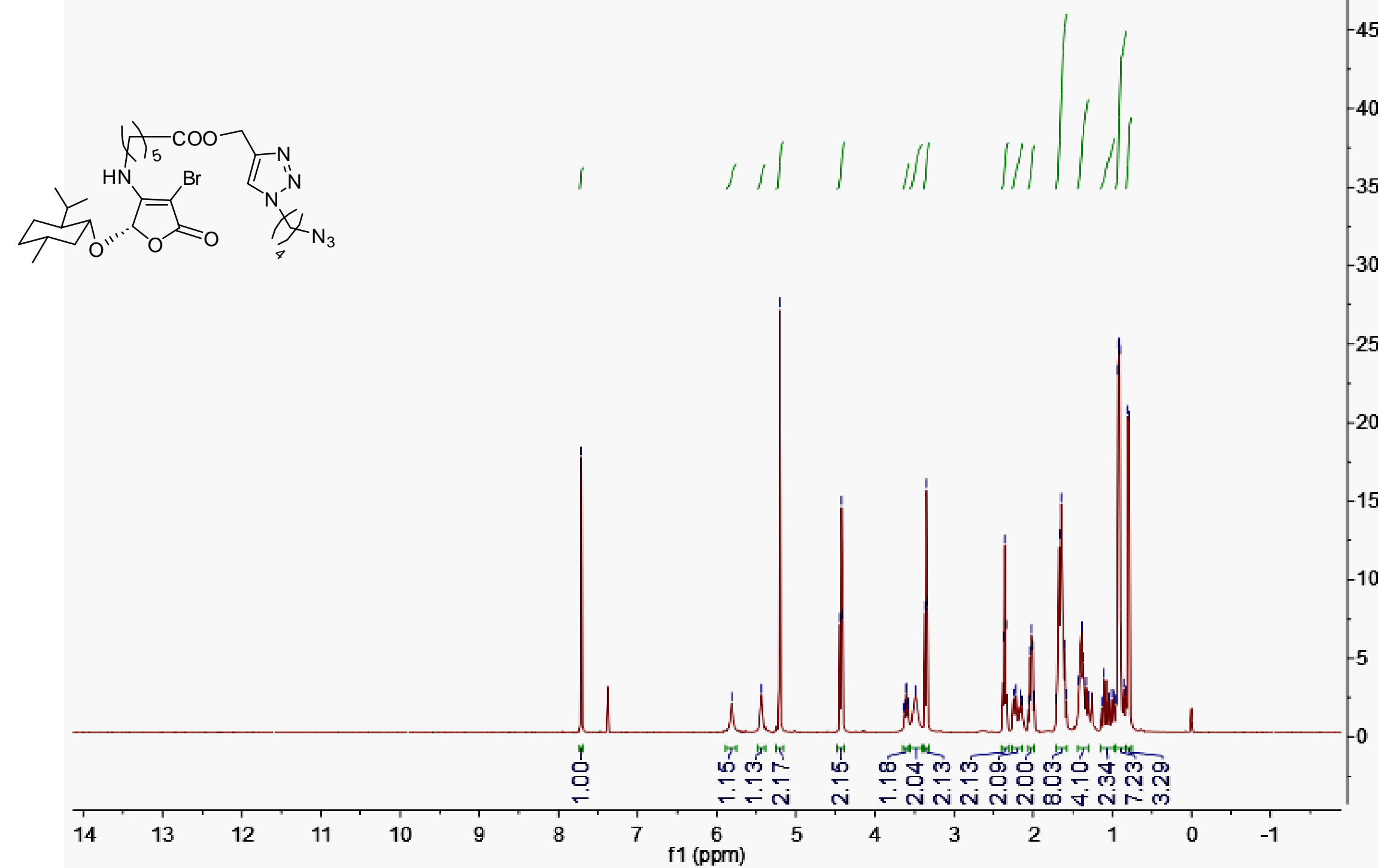


\section{Compound 3c}

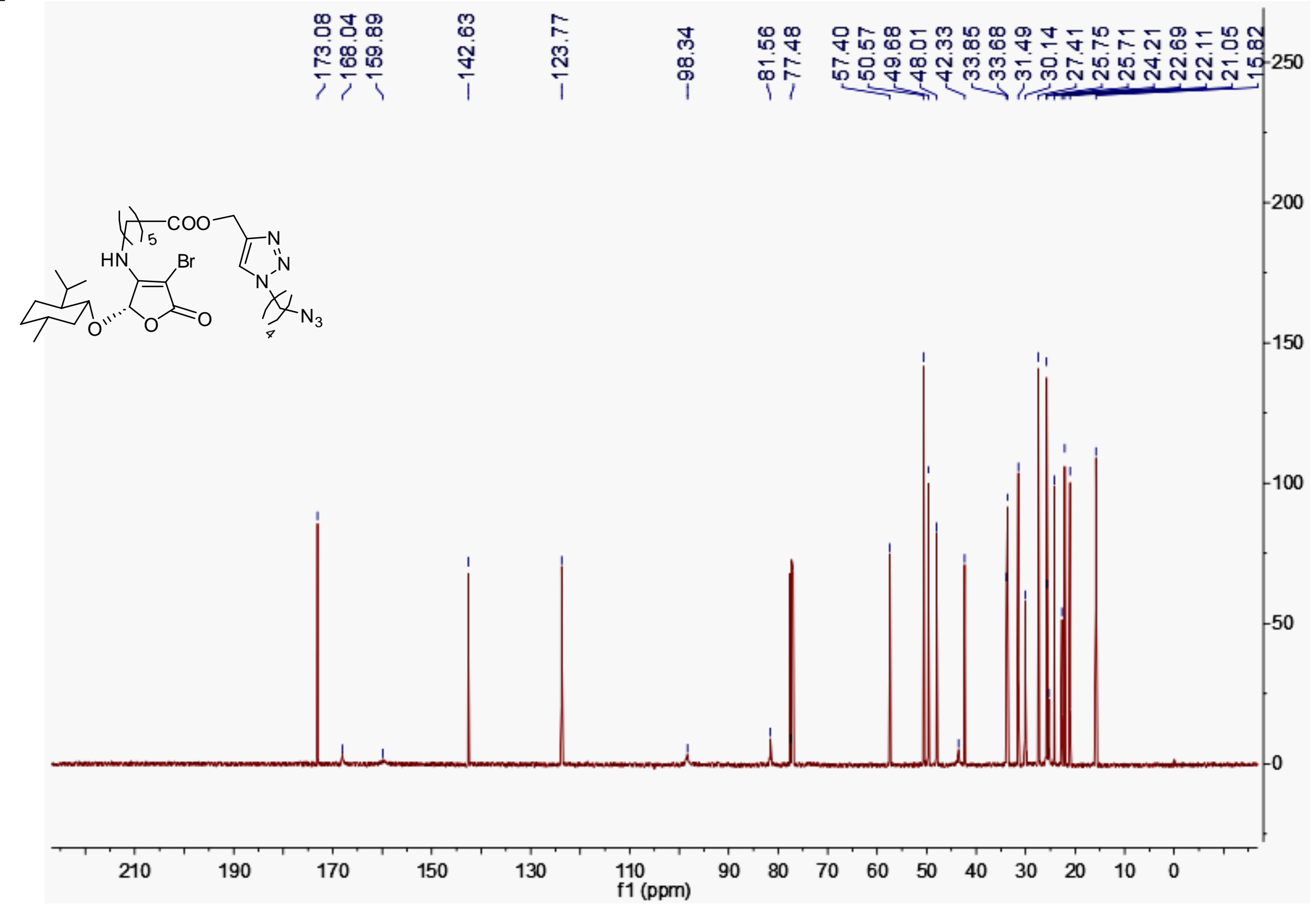




\section{Compound 3d}

ผ

m

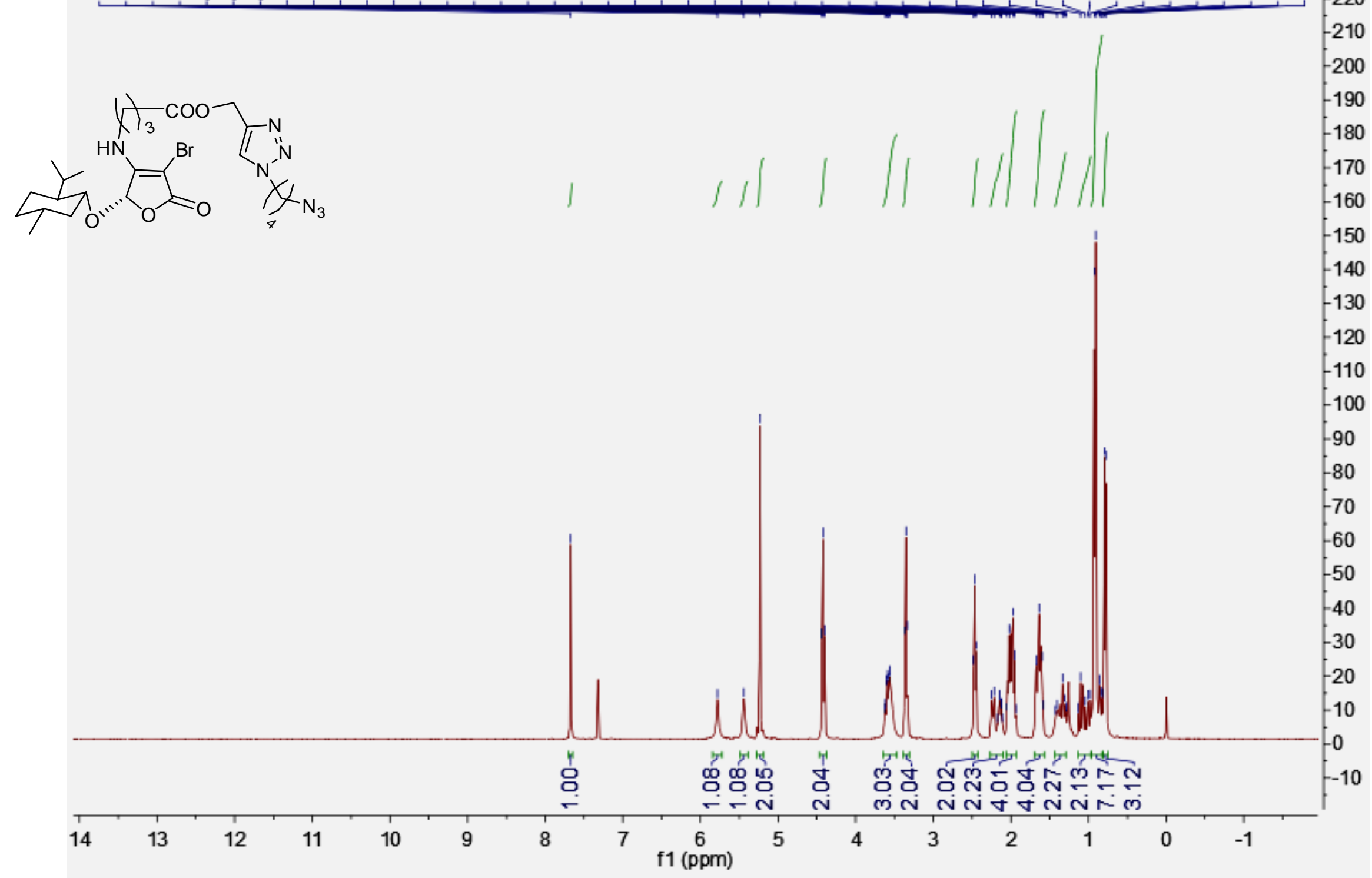




\section{Compound 3d}

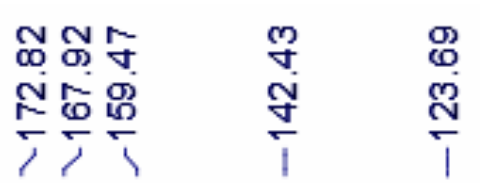

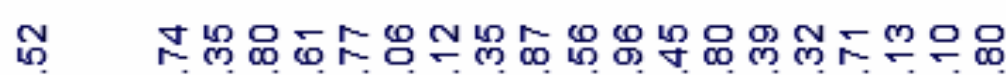

o 11

$-150$

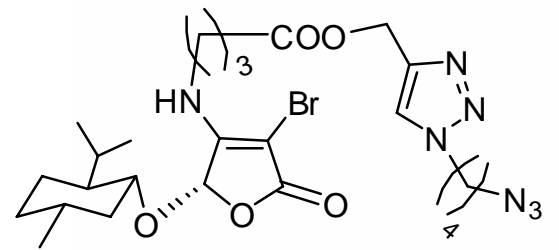




\section{Compound 3e}

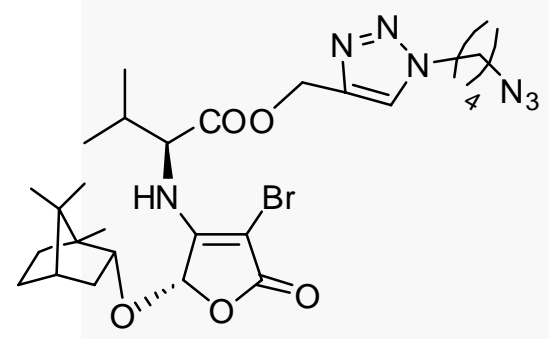

ம

i. ए内

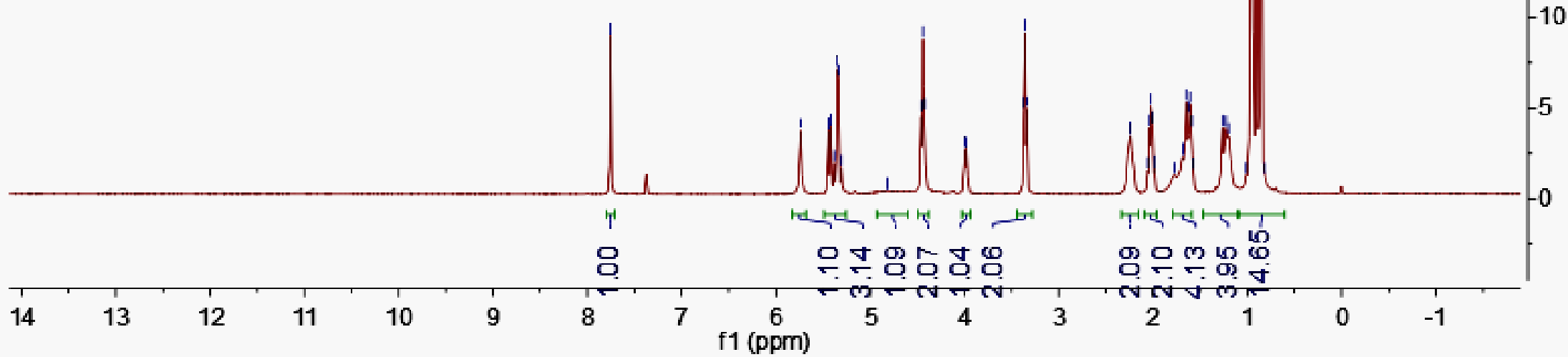




\section{Compound 3 e}

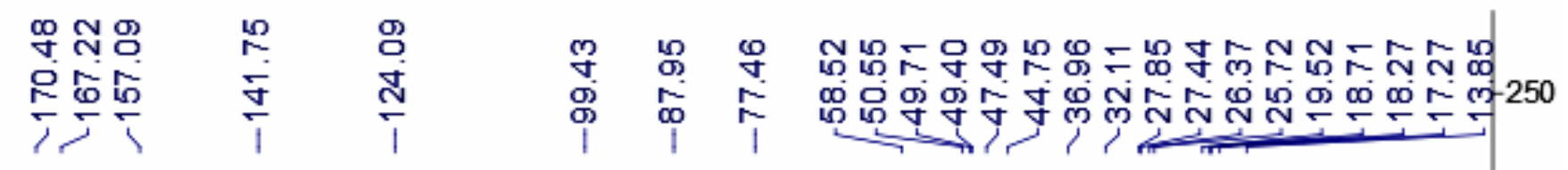
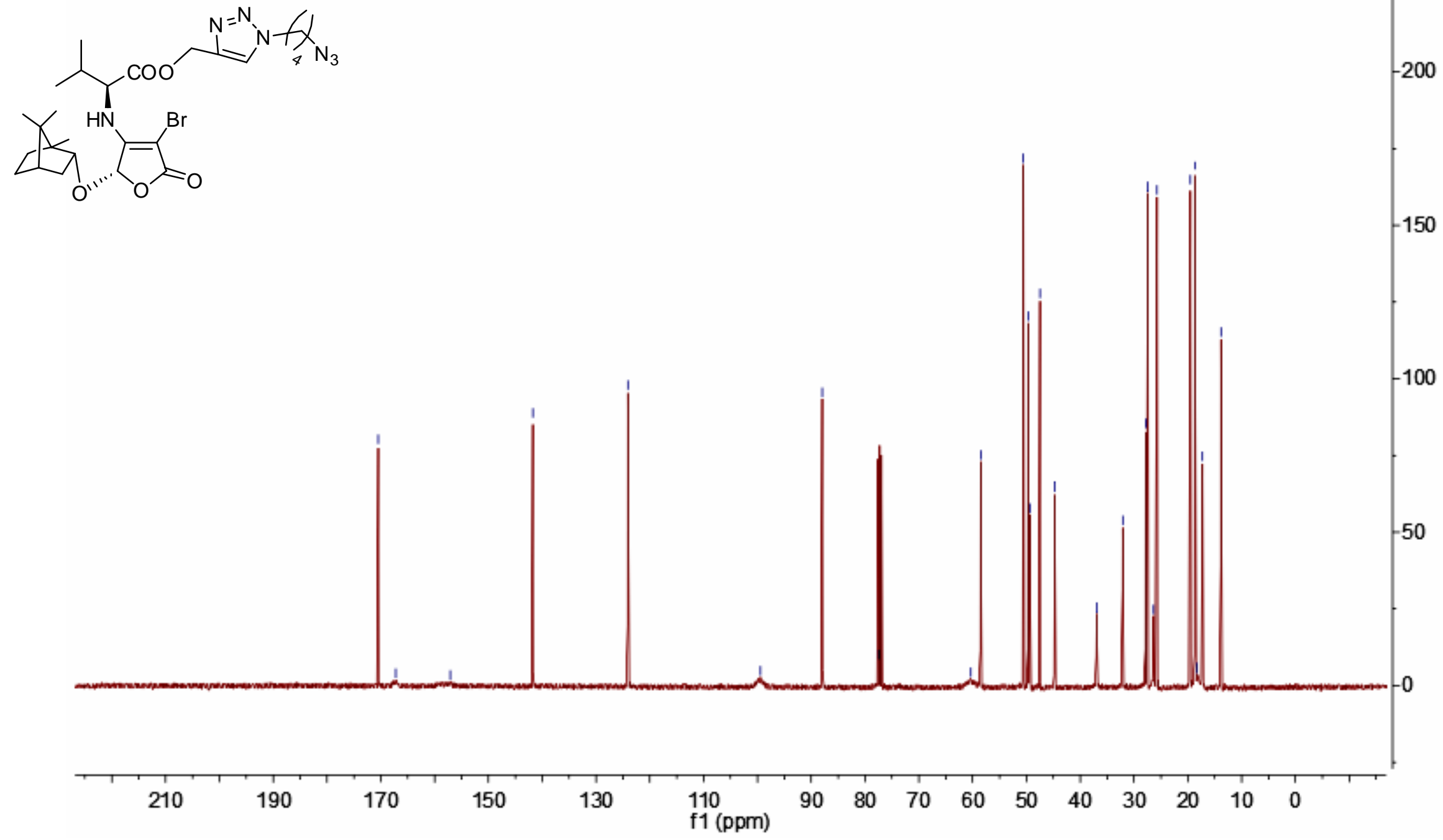


\section{Compound $3 f$}

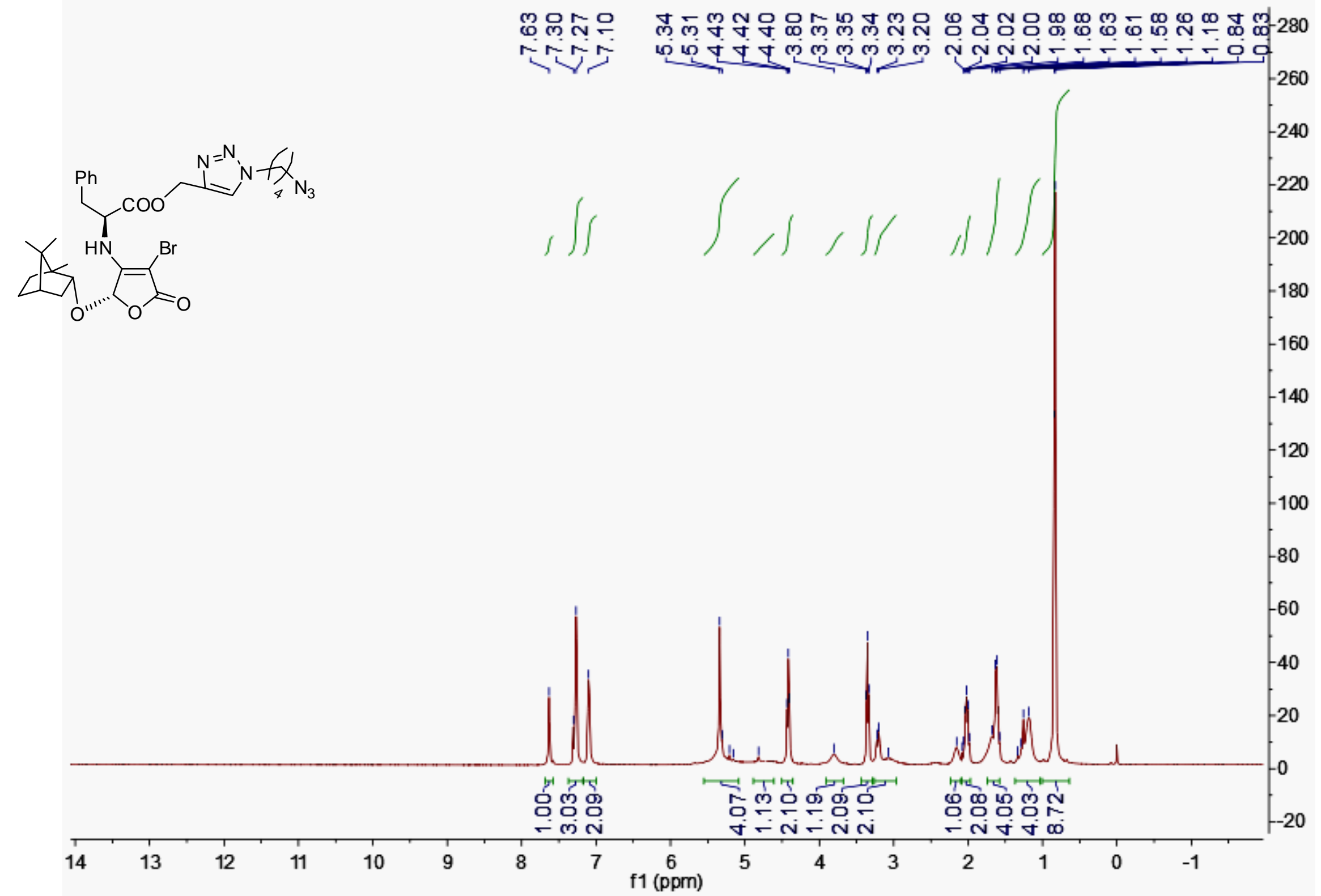




\section{Compound $3 f$}

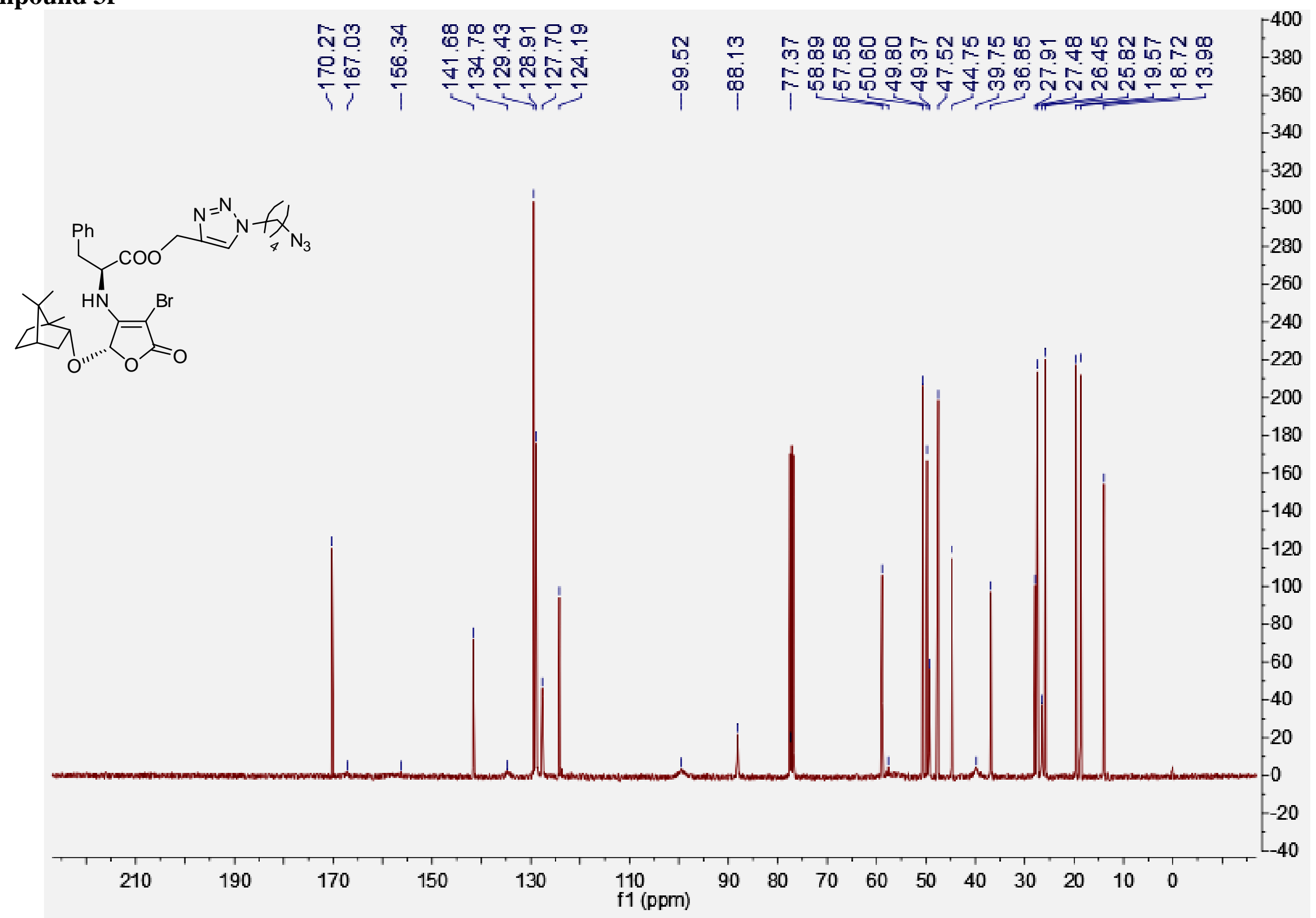




\section{Compound 3g}

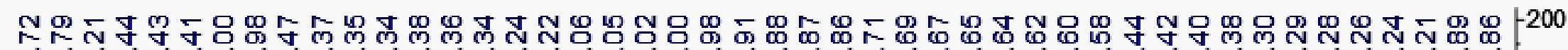

m is
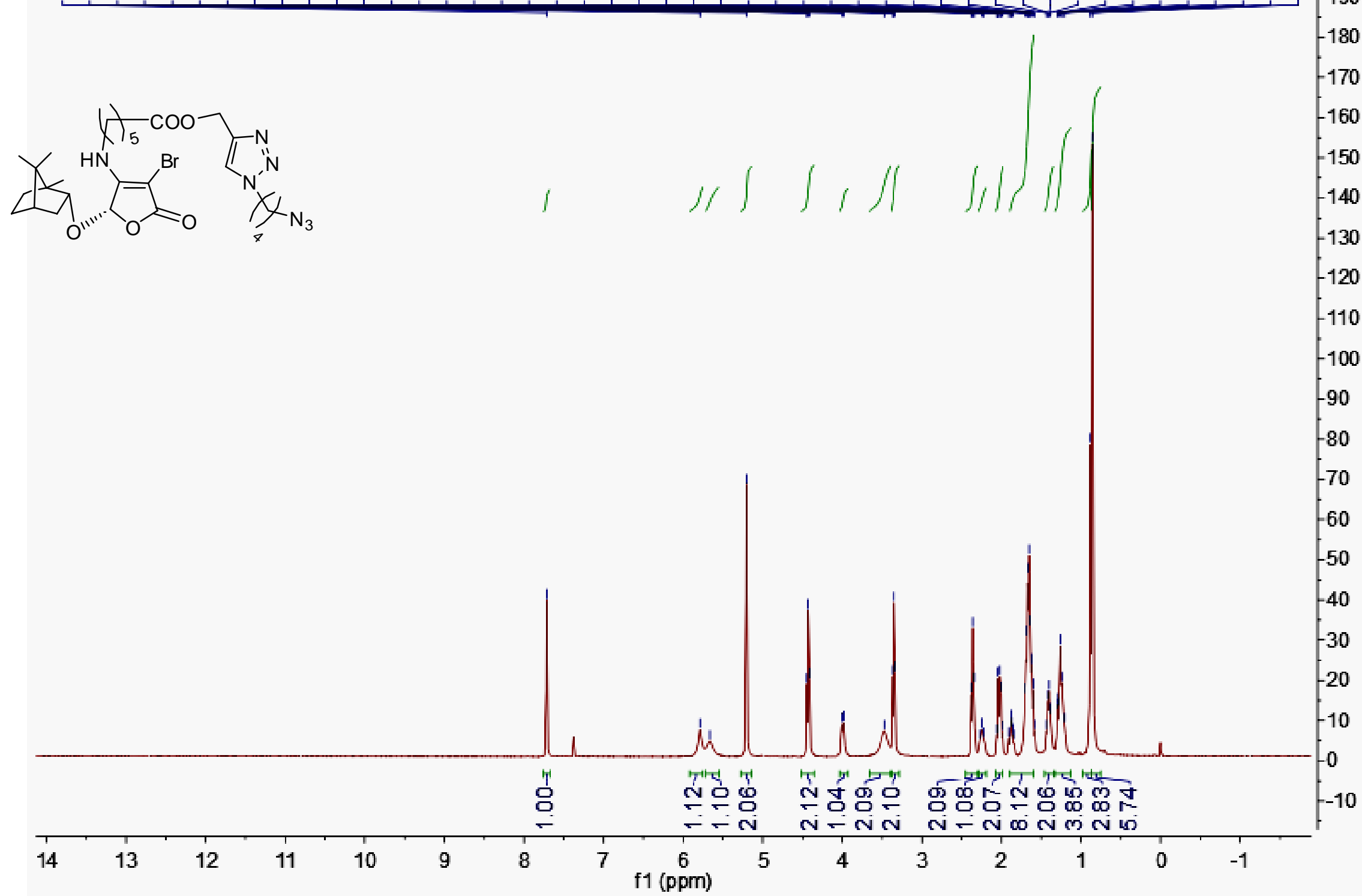


\section{Compound 3g}

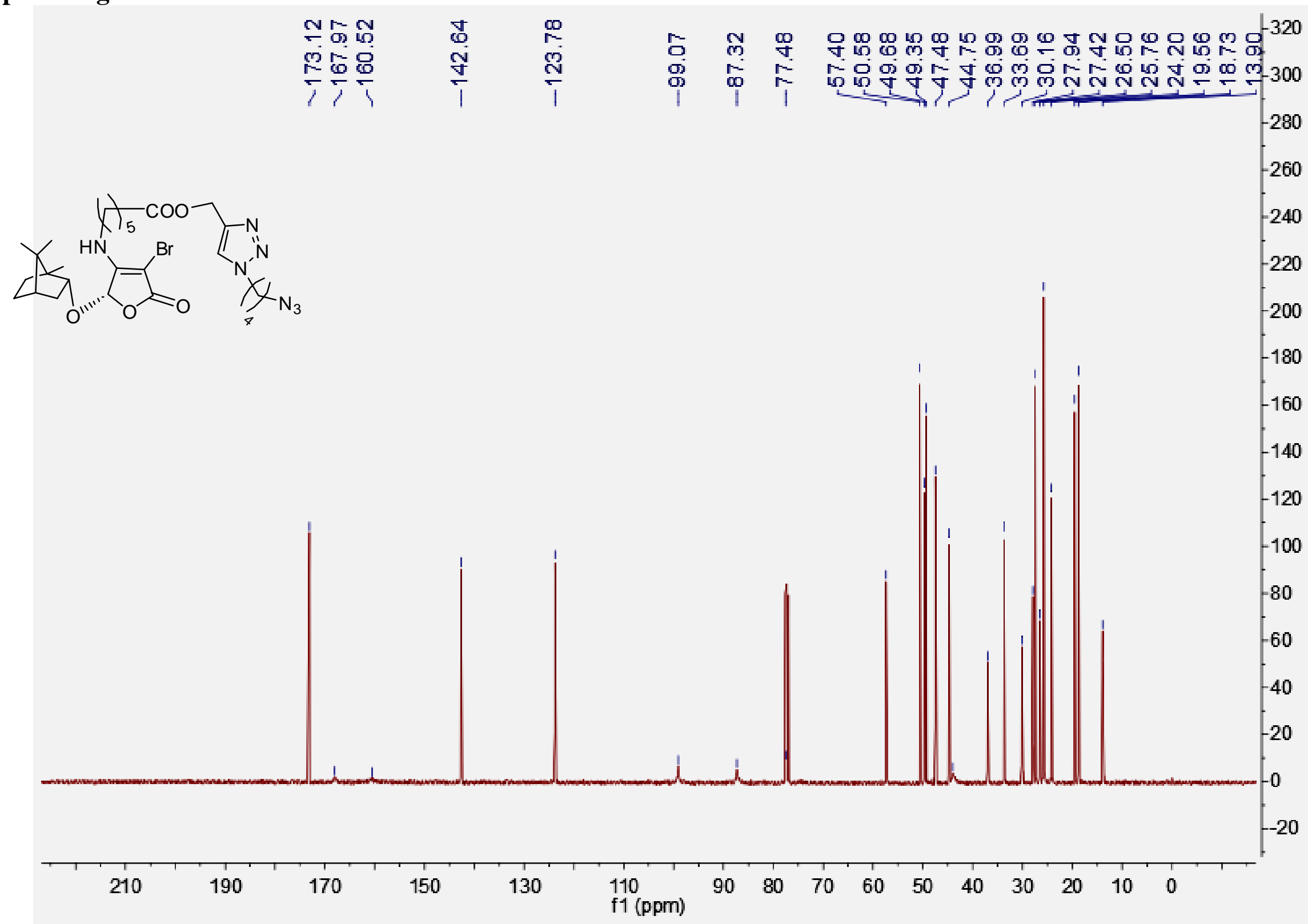




\section{Compound $3 h$}

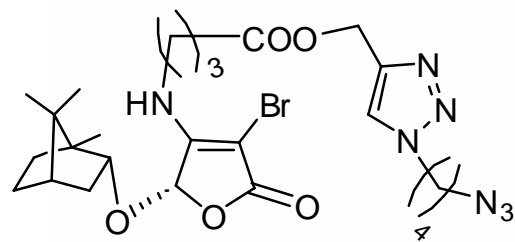

NNM 寸

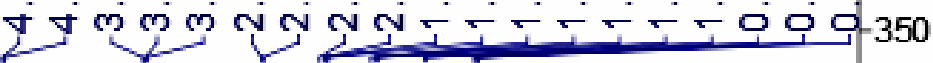

O"
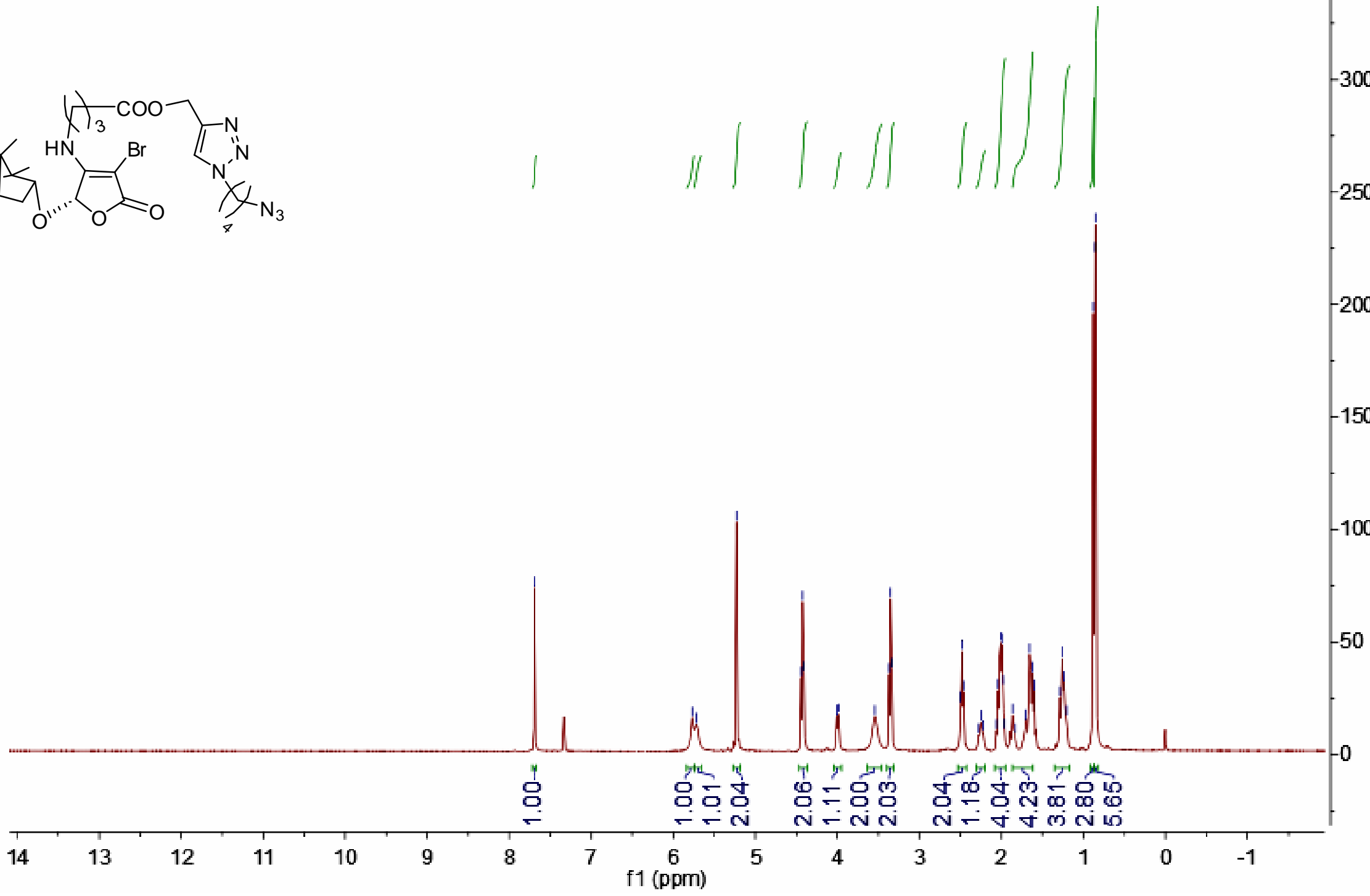


\section{Compound $3 h$}

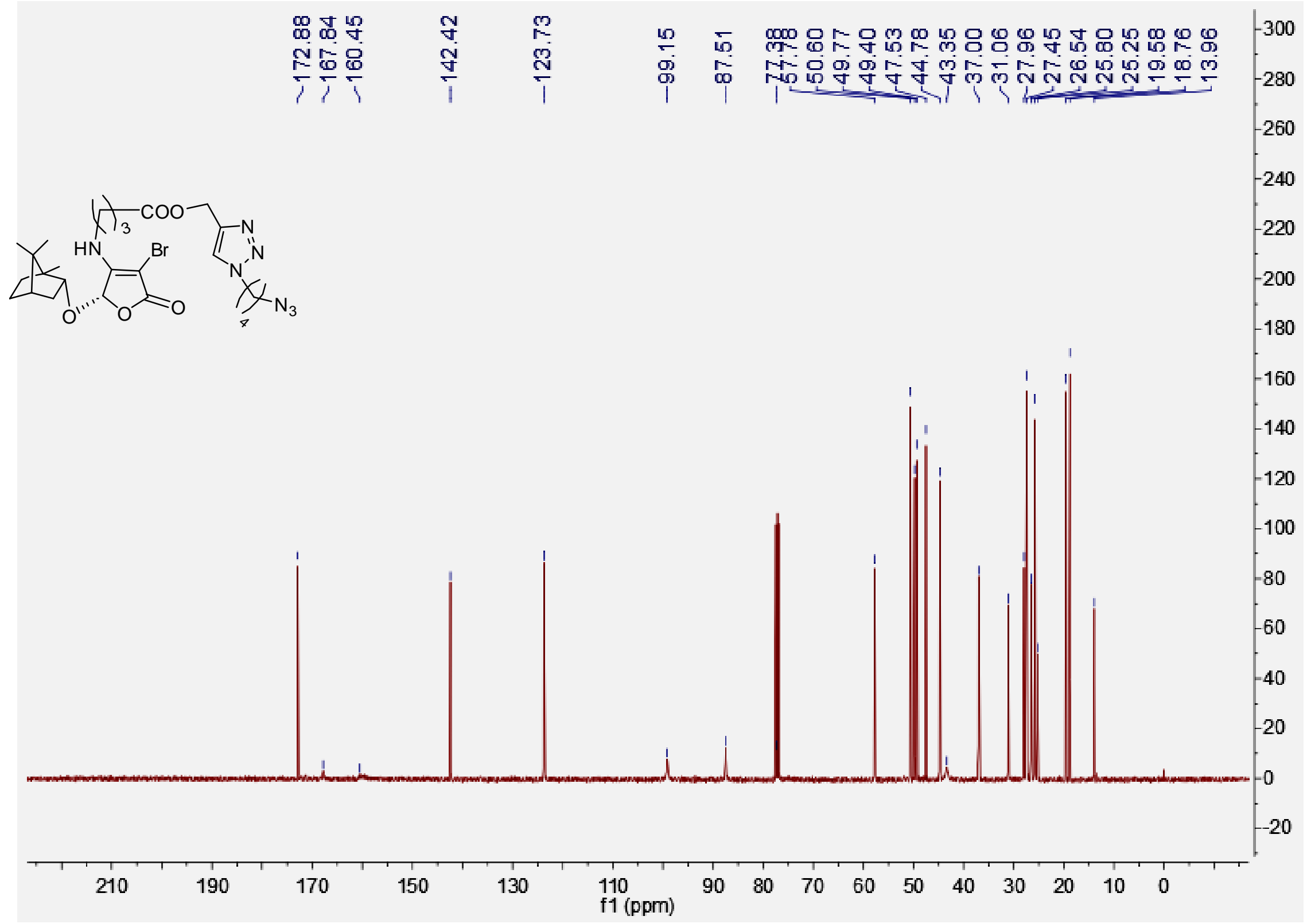




\section{Compound 5aa}

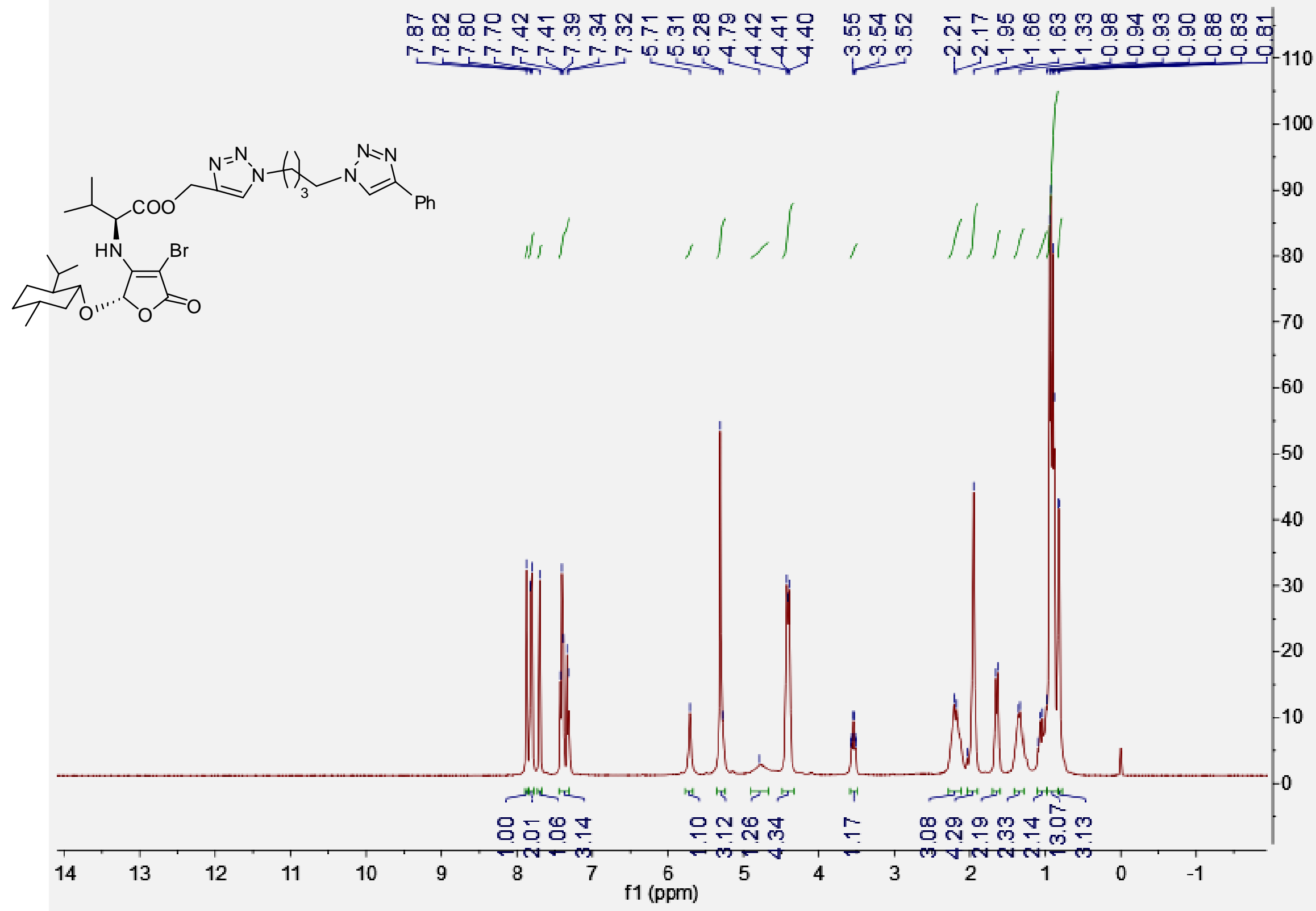




\section{Compound 5aa}

オ以

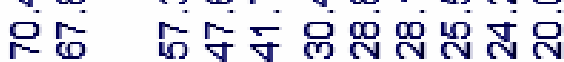

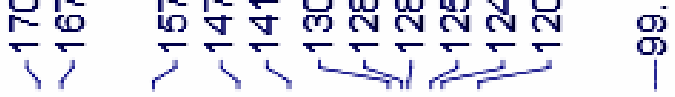

$\stackrel{2}{\infty}$

240

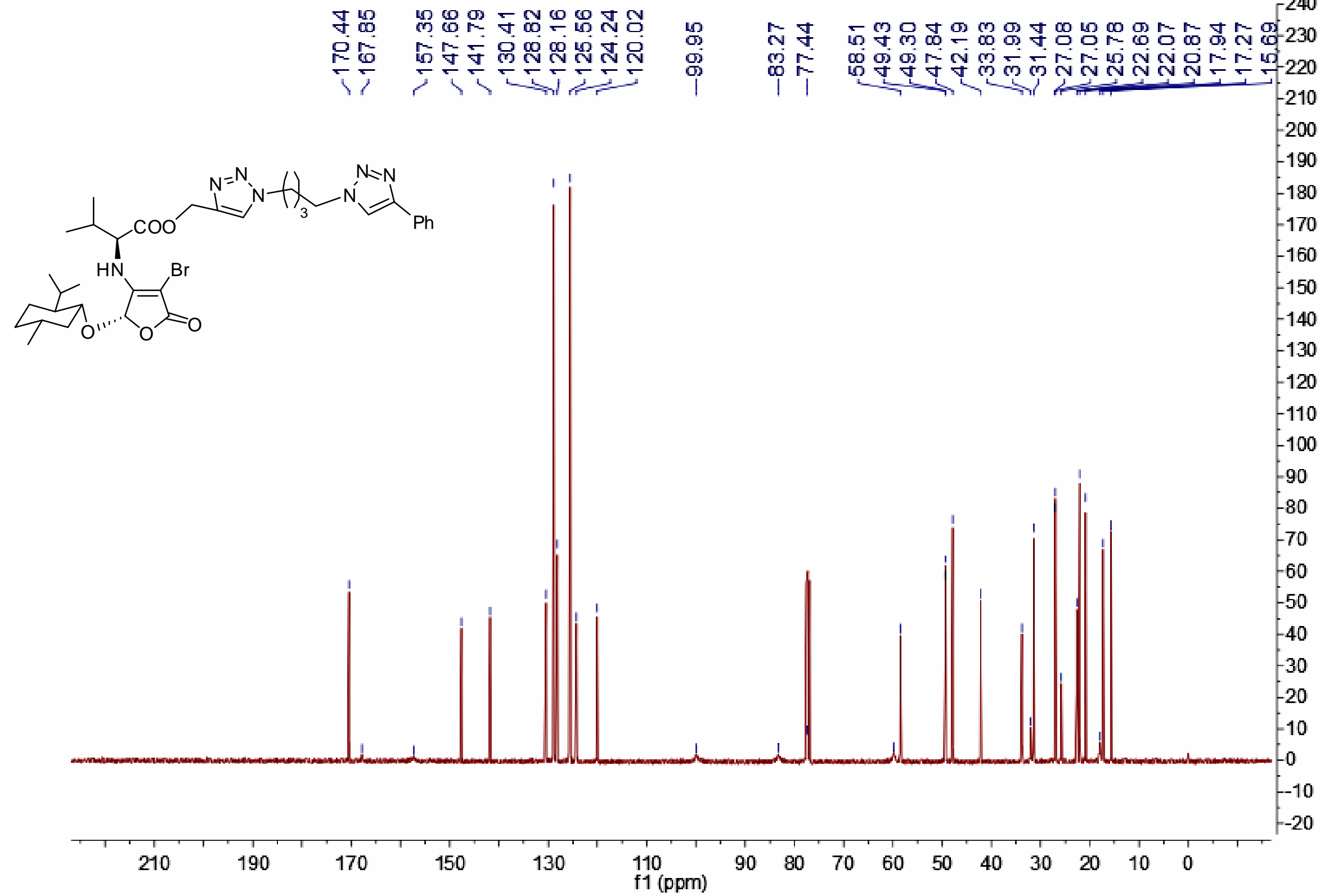




\section{Compound 5ab}

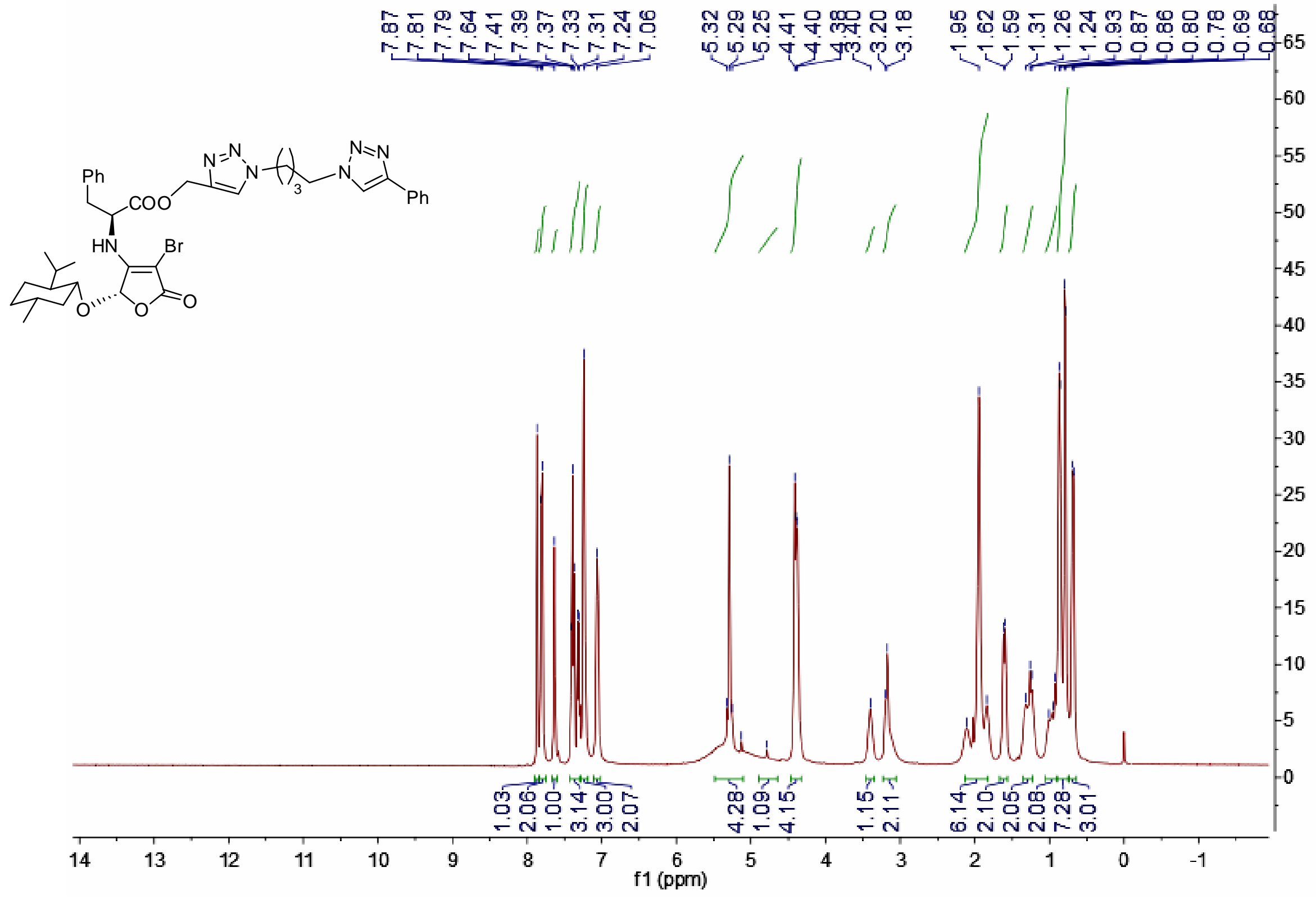




\section{Compound 5ab}

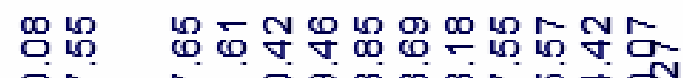

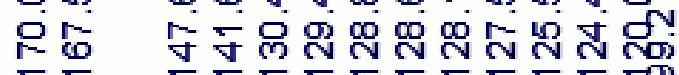

ธ ธํำ

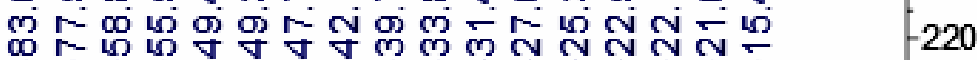

i)
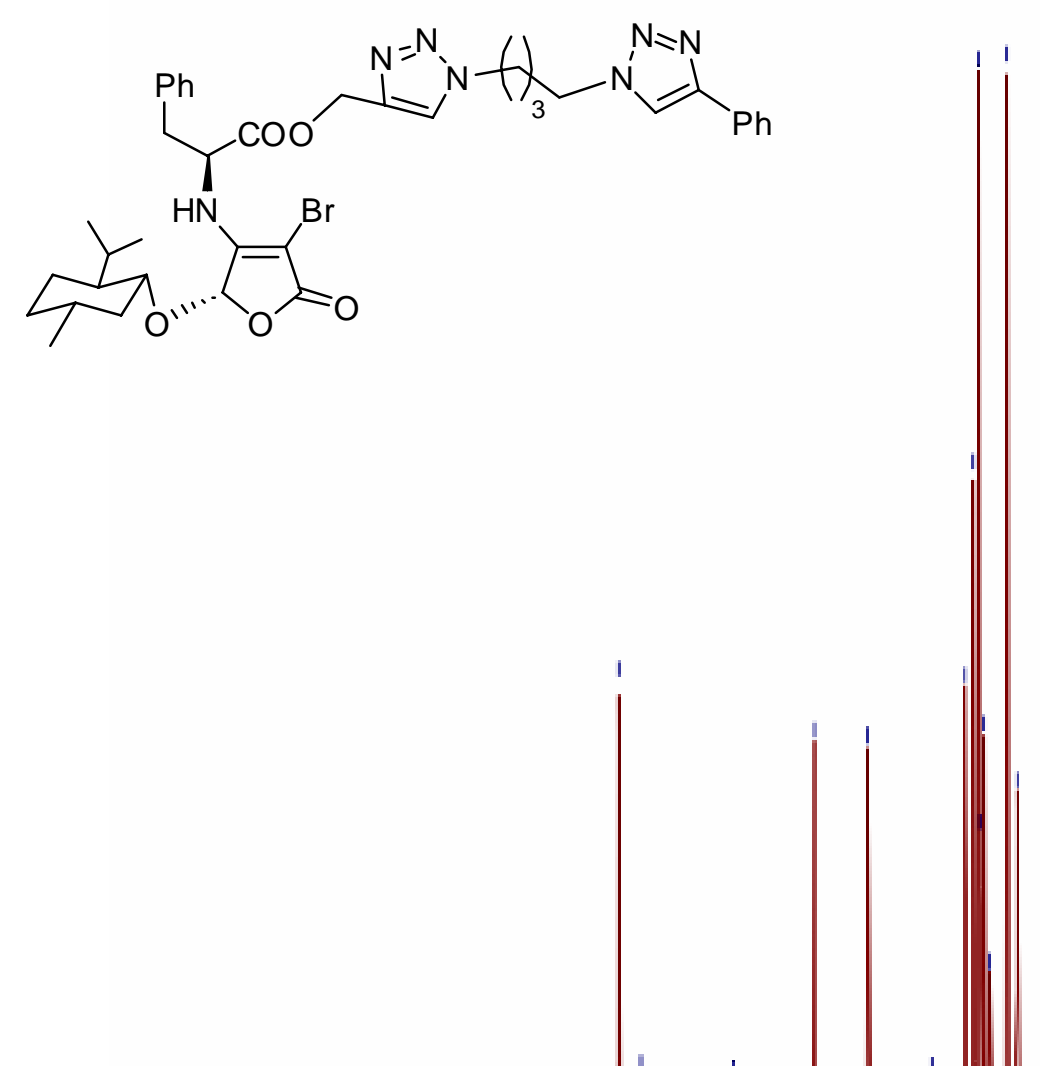

180

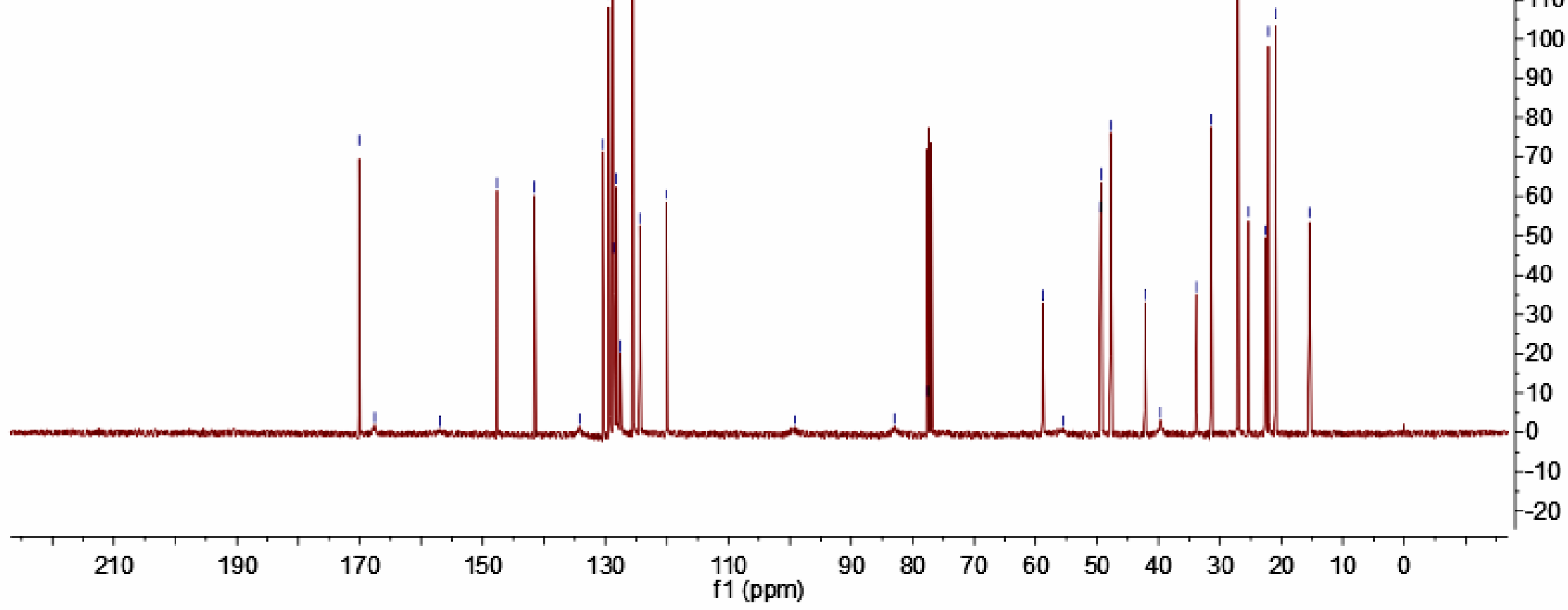




\section{Compound 5 ac}

ఇ య

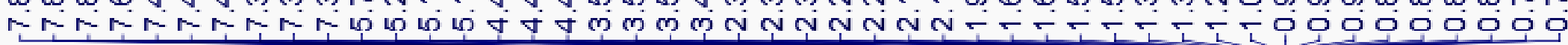

(1)
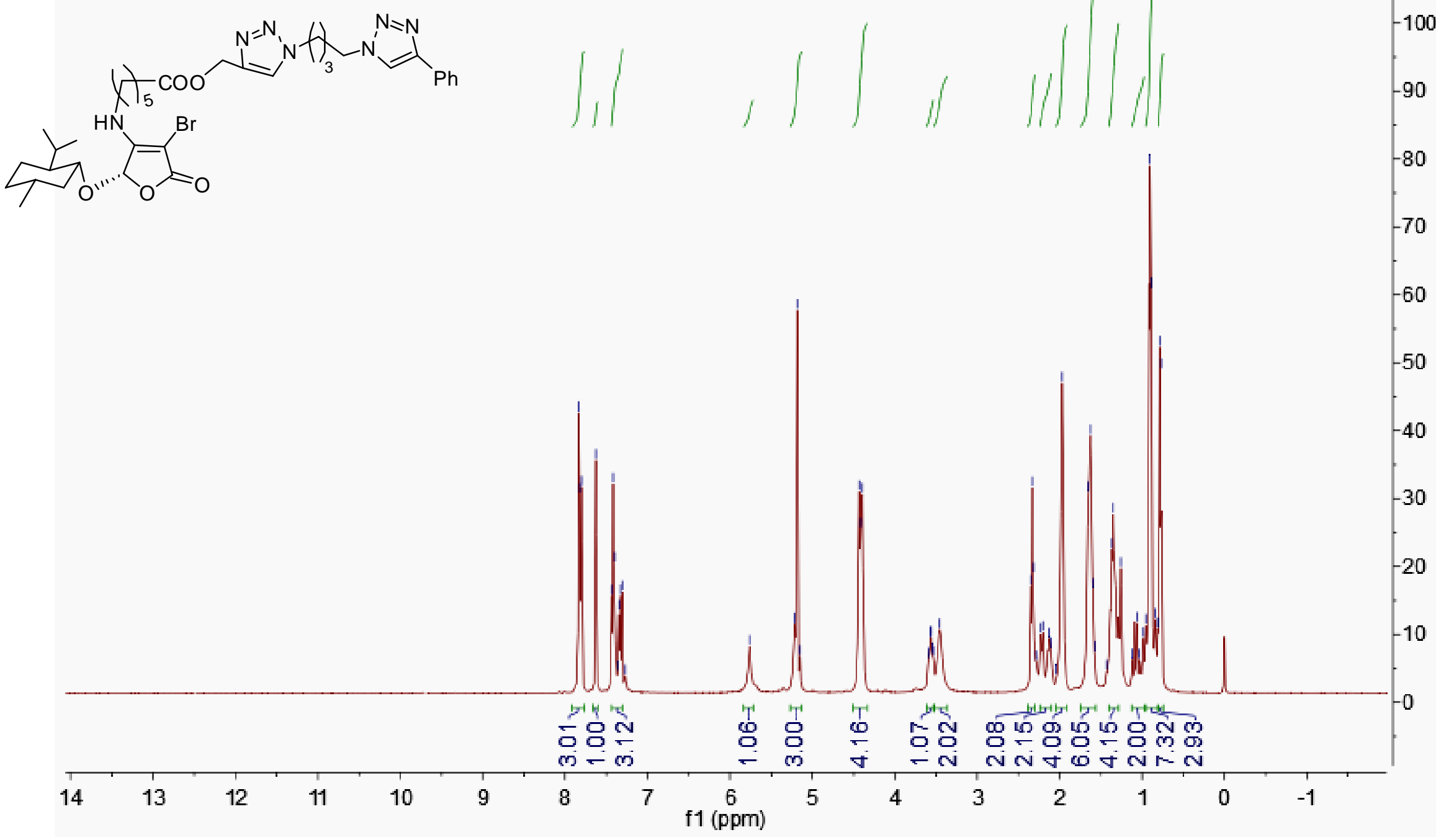


\section{Compound 5ac}

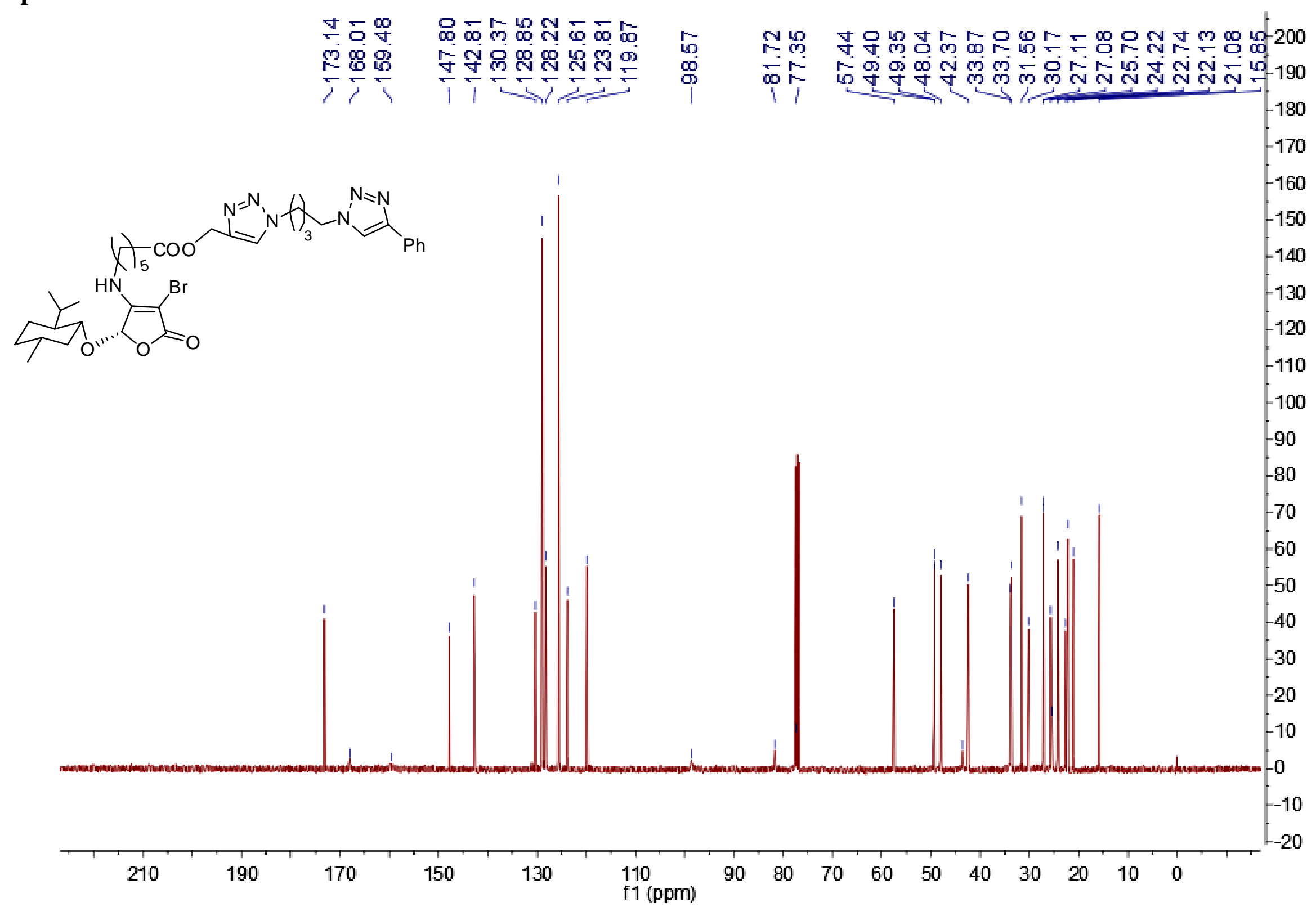




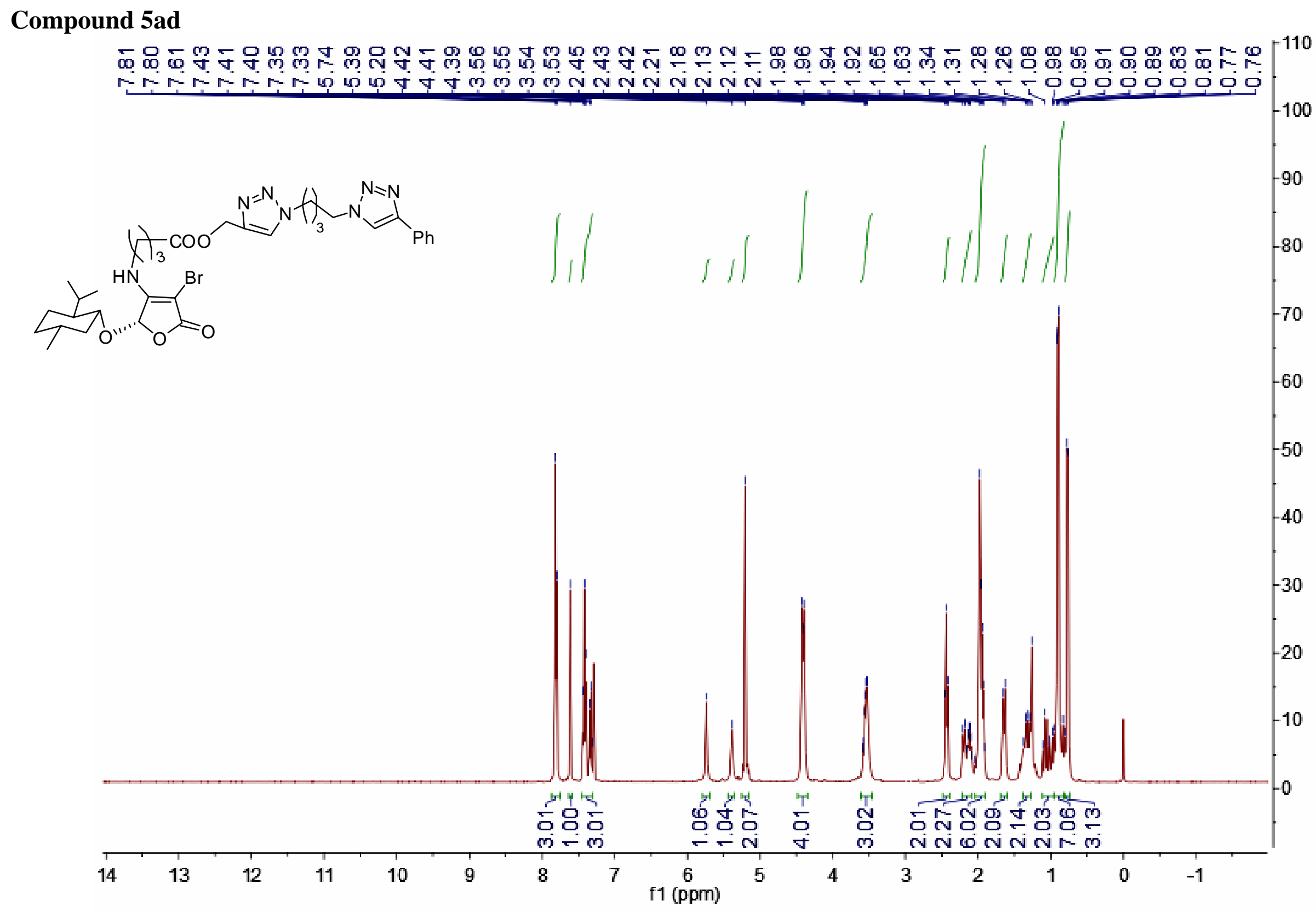




\section{Compound 5ad}

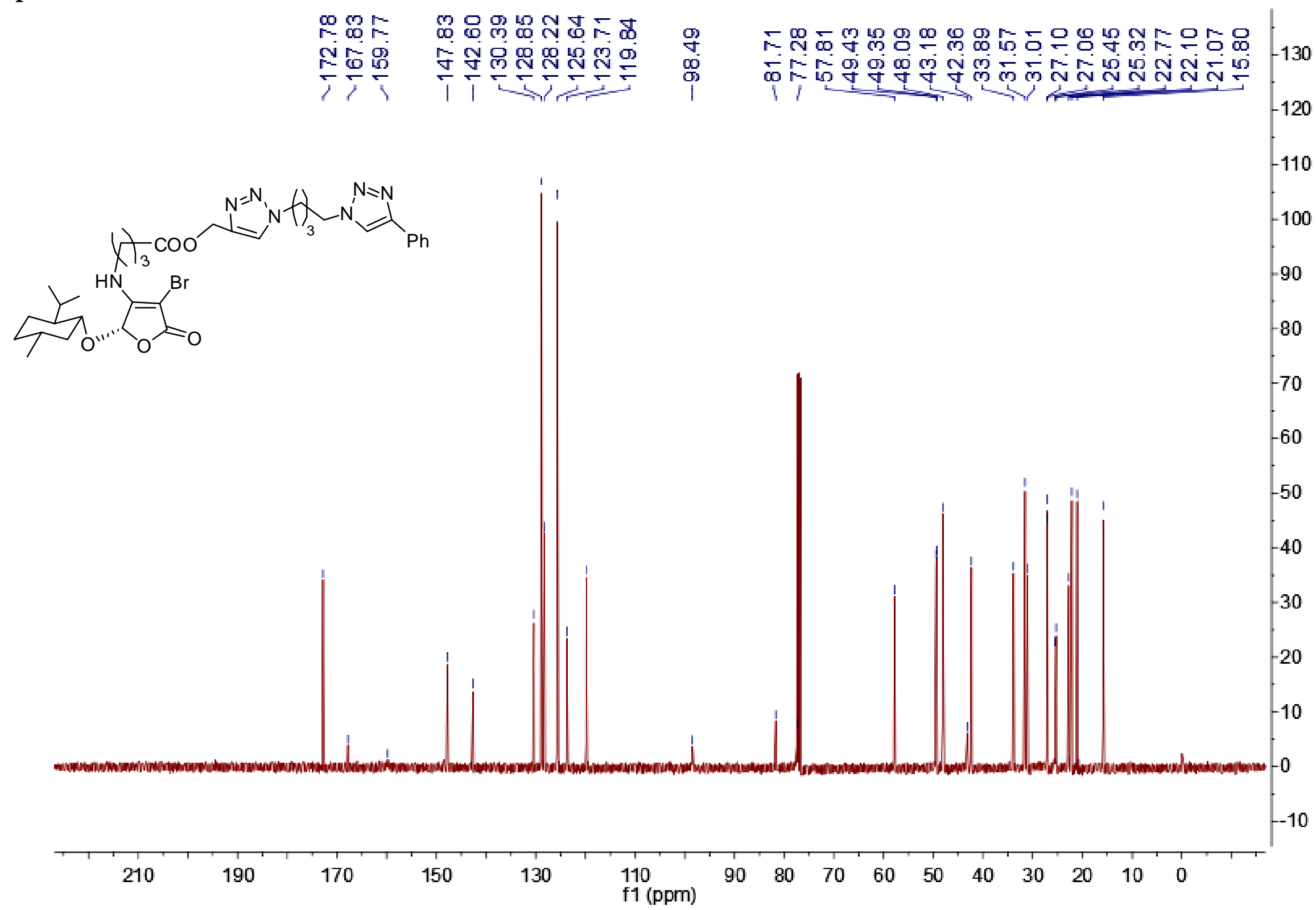




\section{Compound $5 a e$}

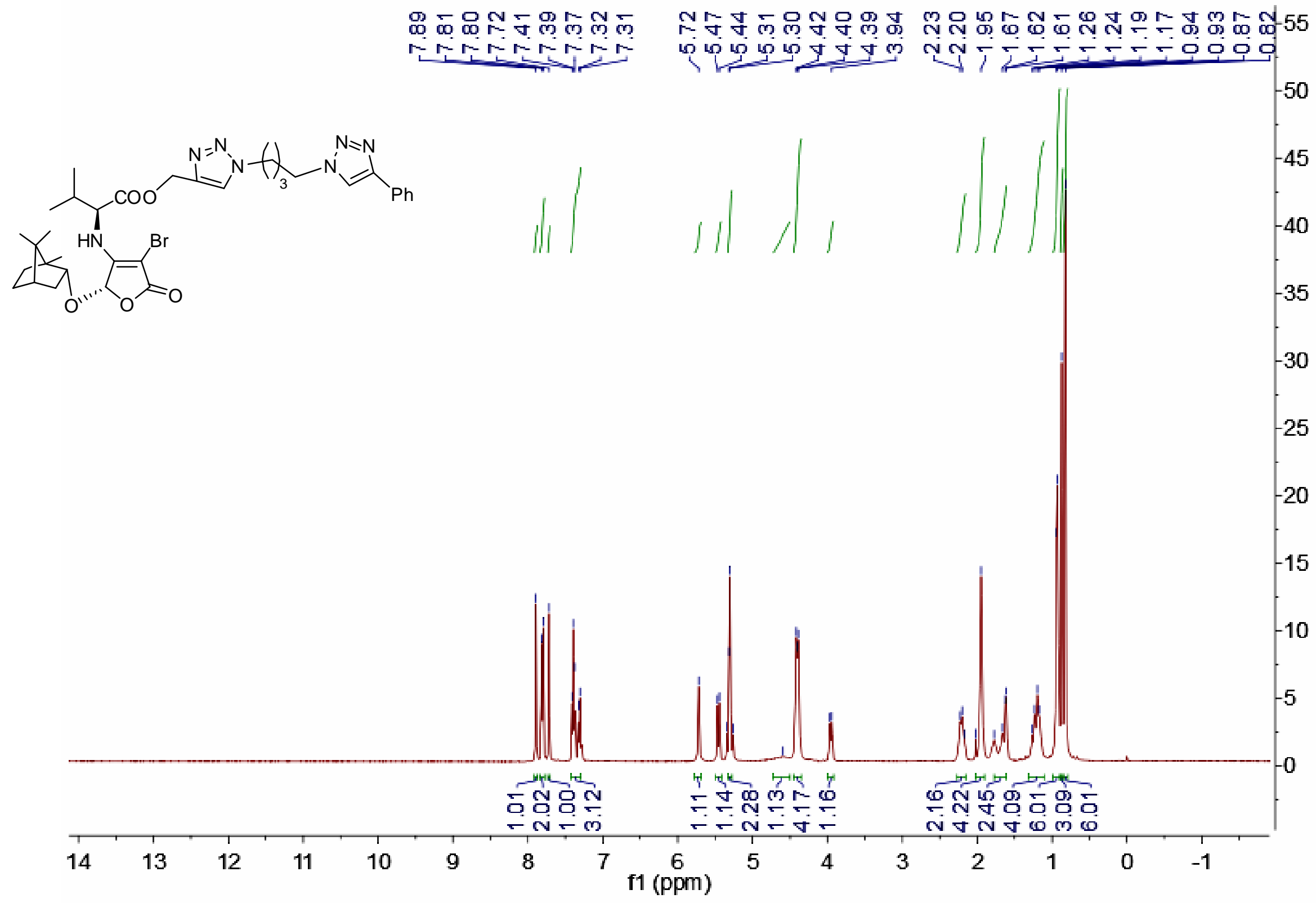




\section{Compound 5ae}

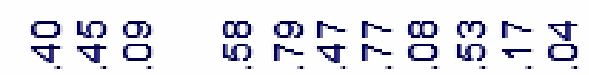

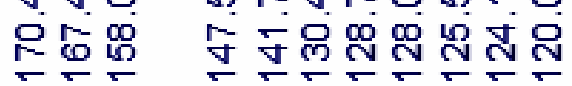

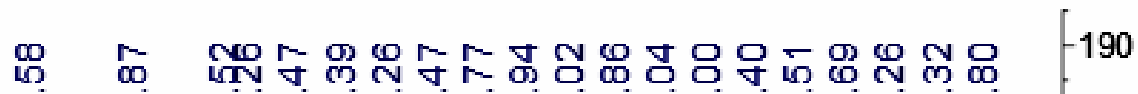

क

7) 1111
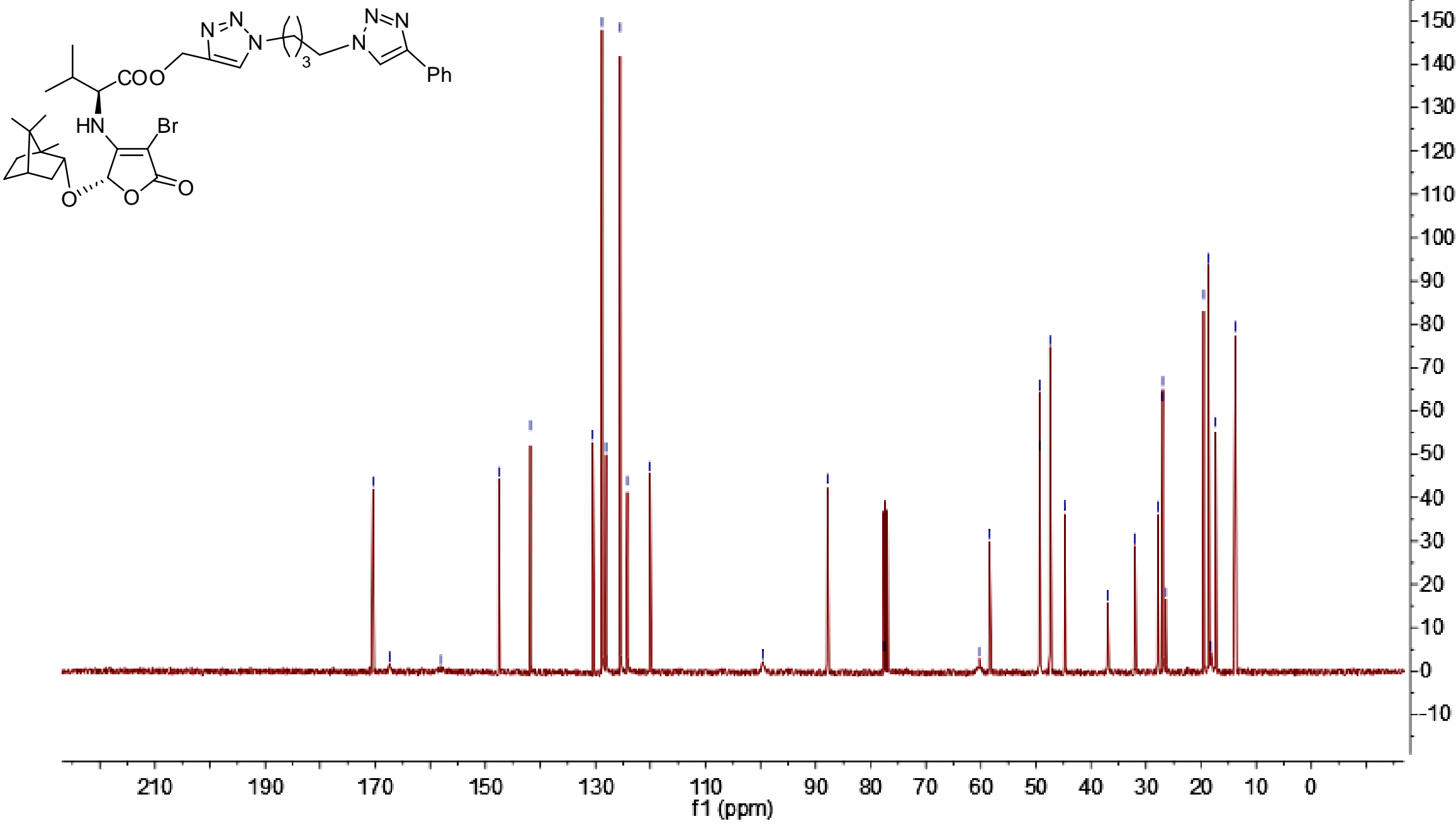


\section{Compound 5af}

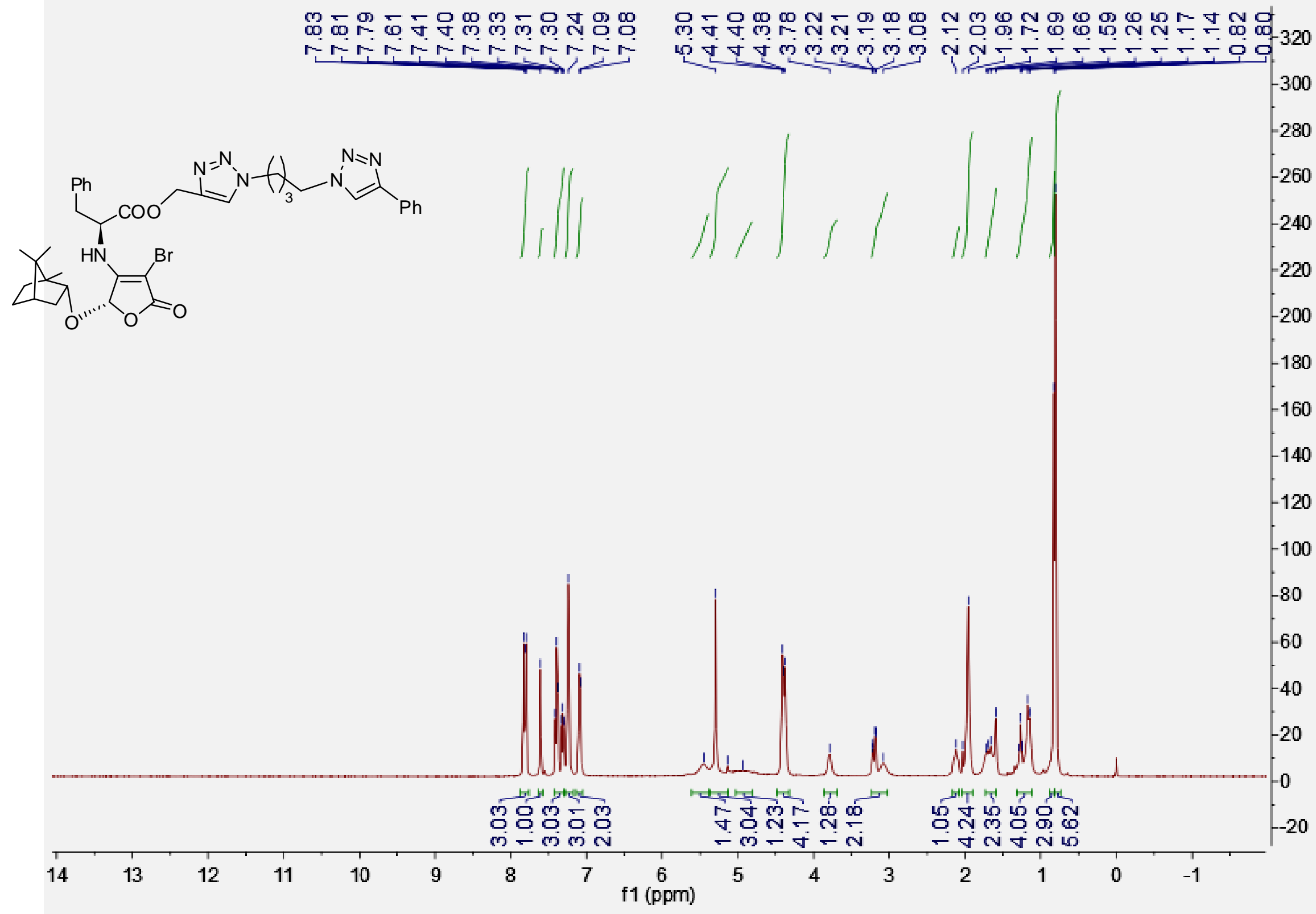




\section{Compound 5af}

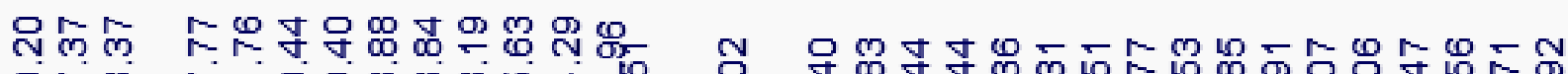

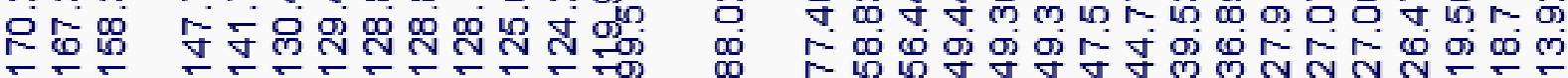

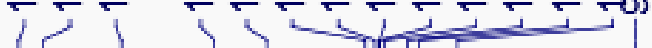
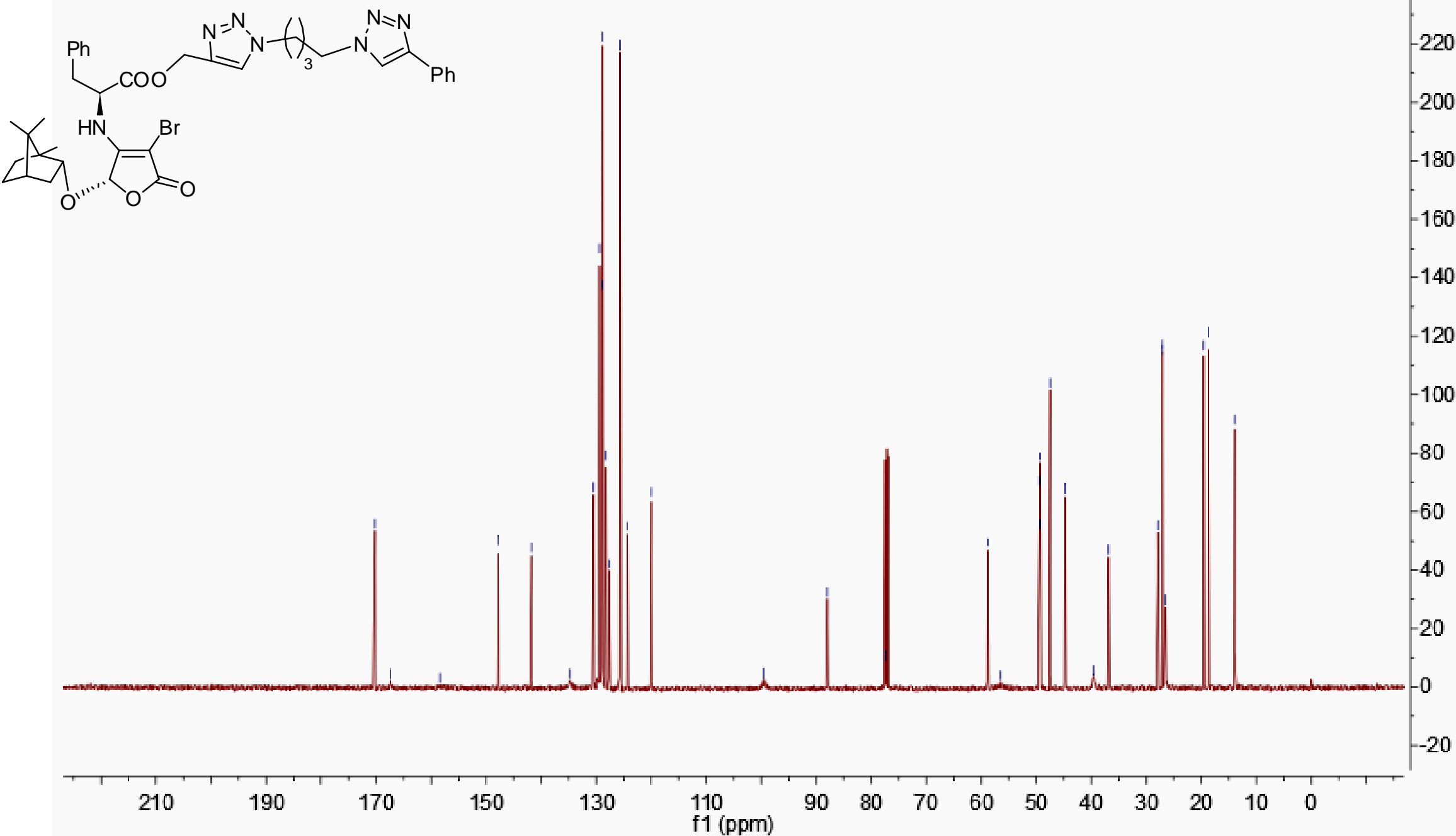


\section{Compound 5ag}

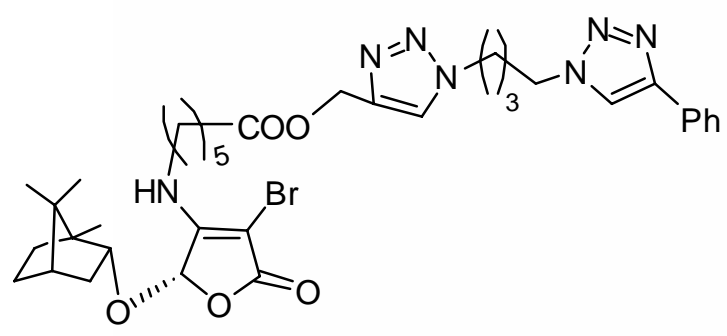

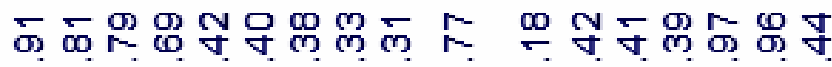

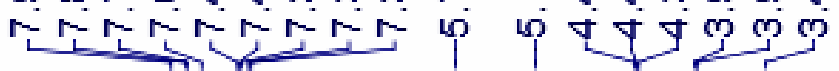

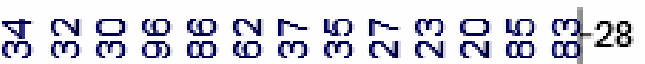

ن

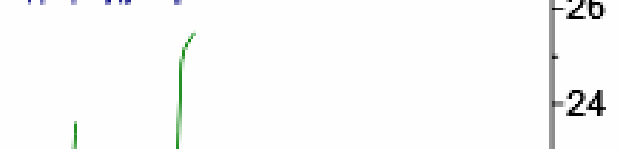

24

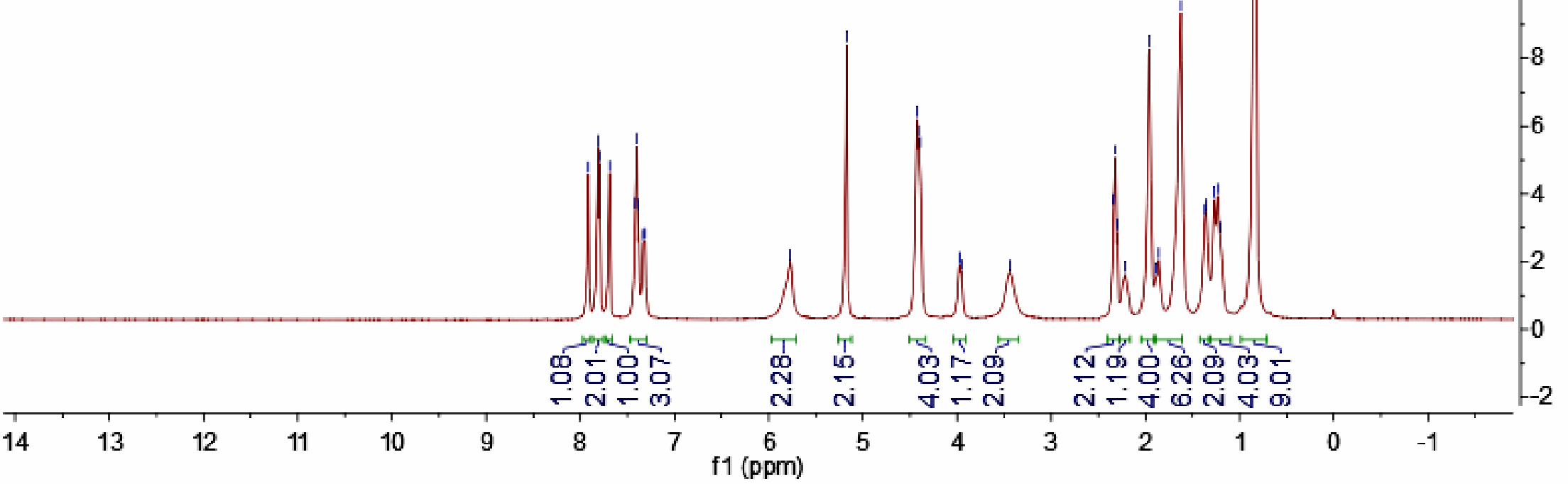




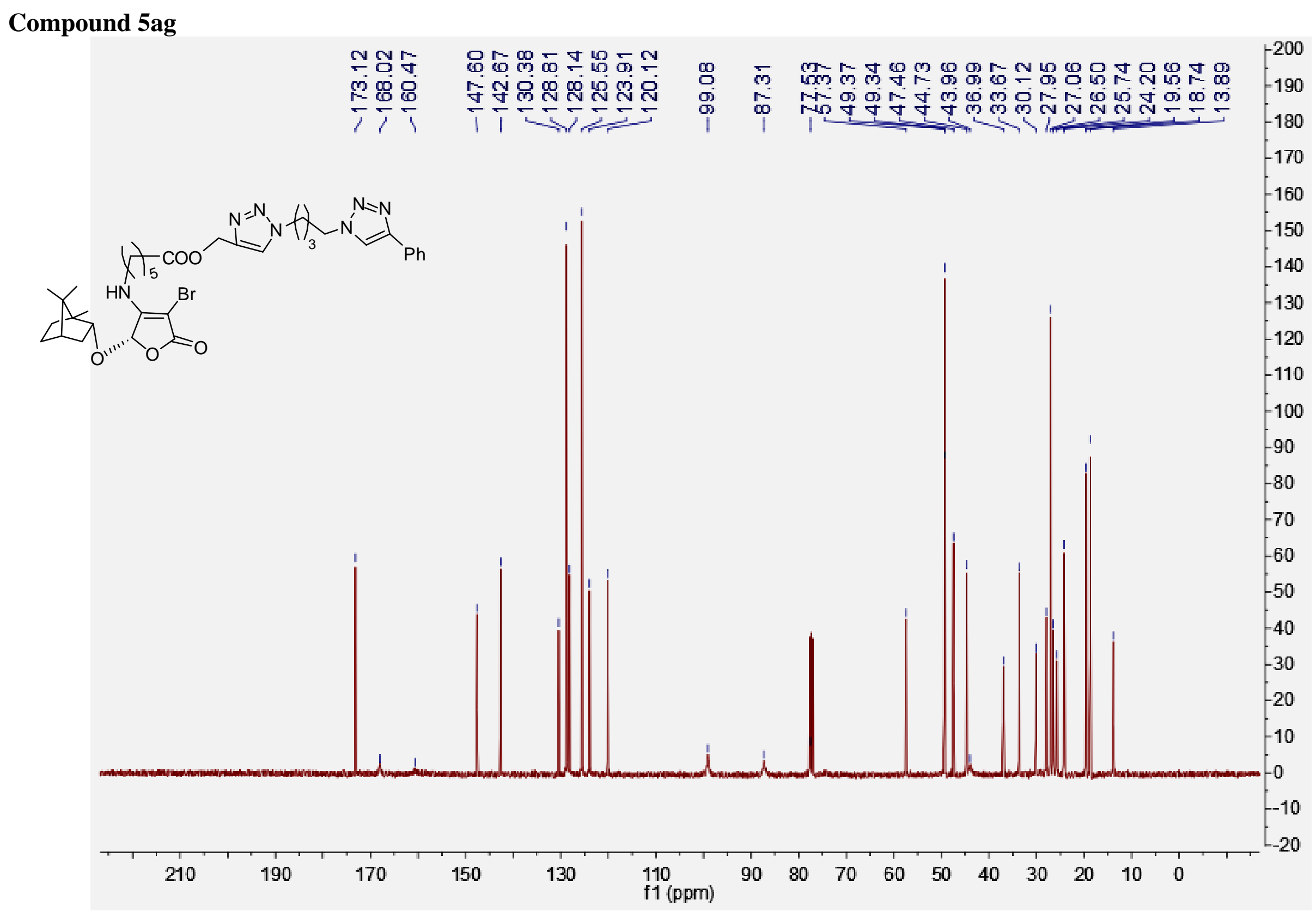




\section{Compound 5ah}

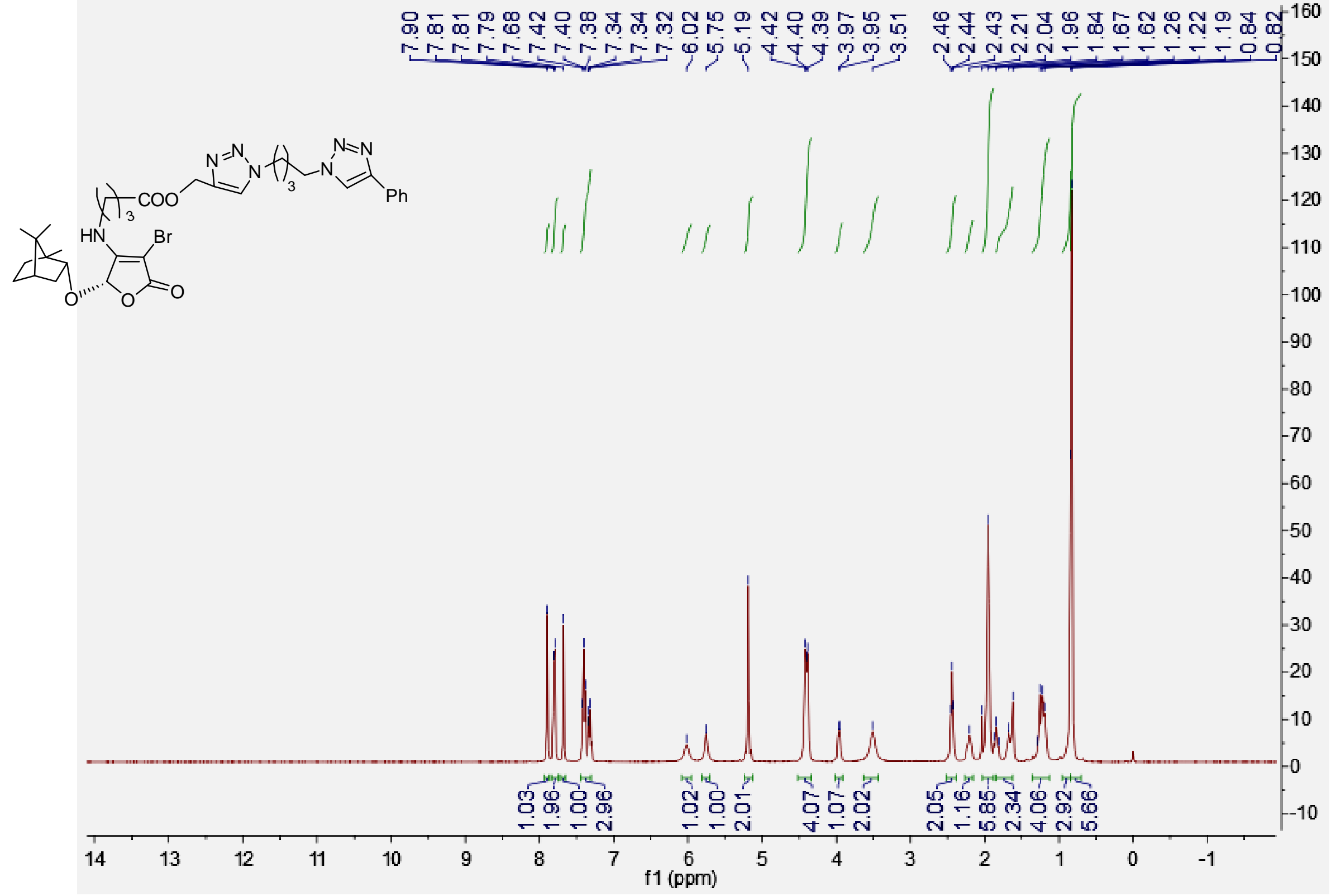




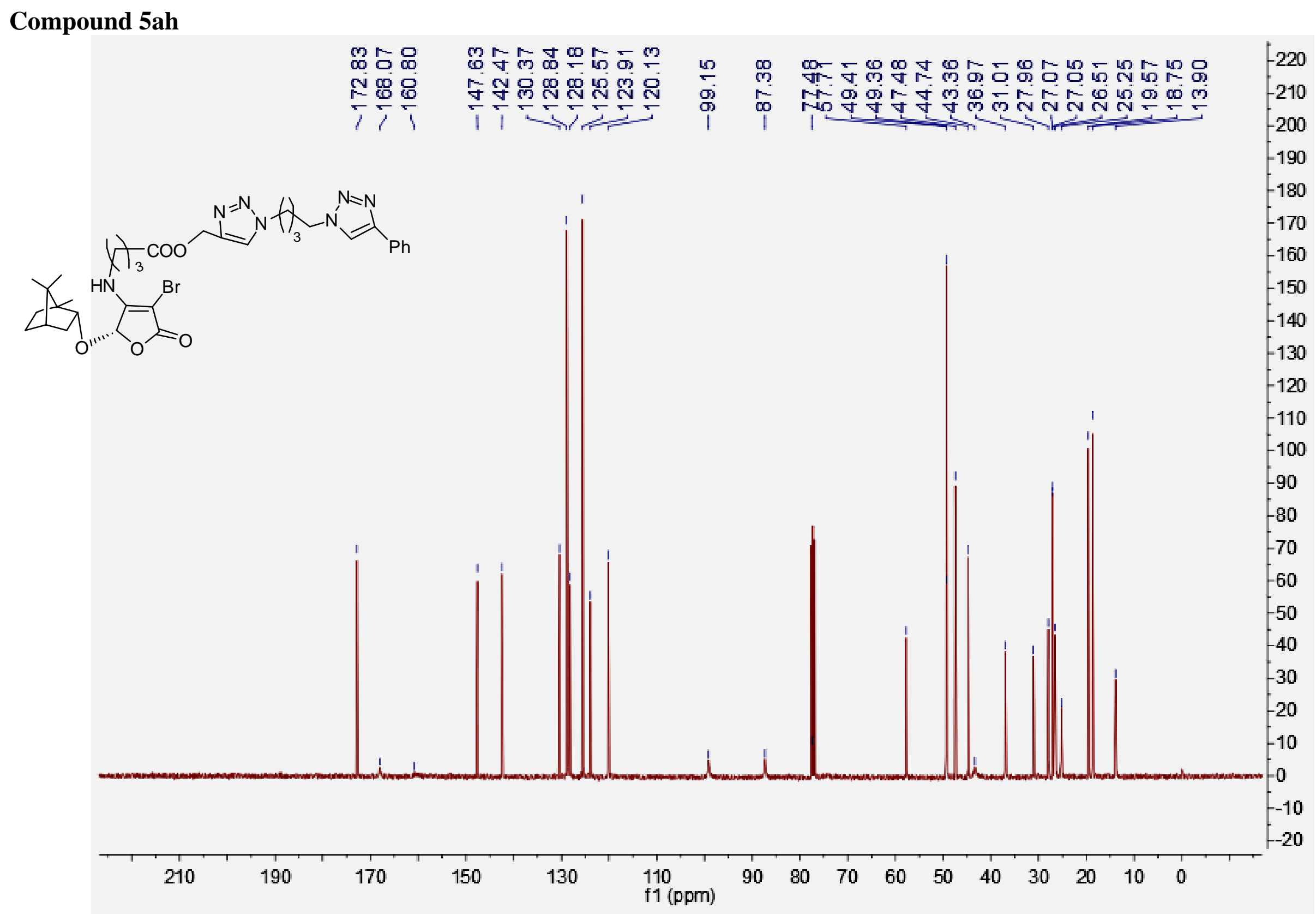




\section{Compound 5ba}

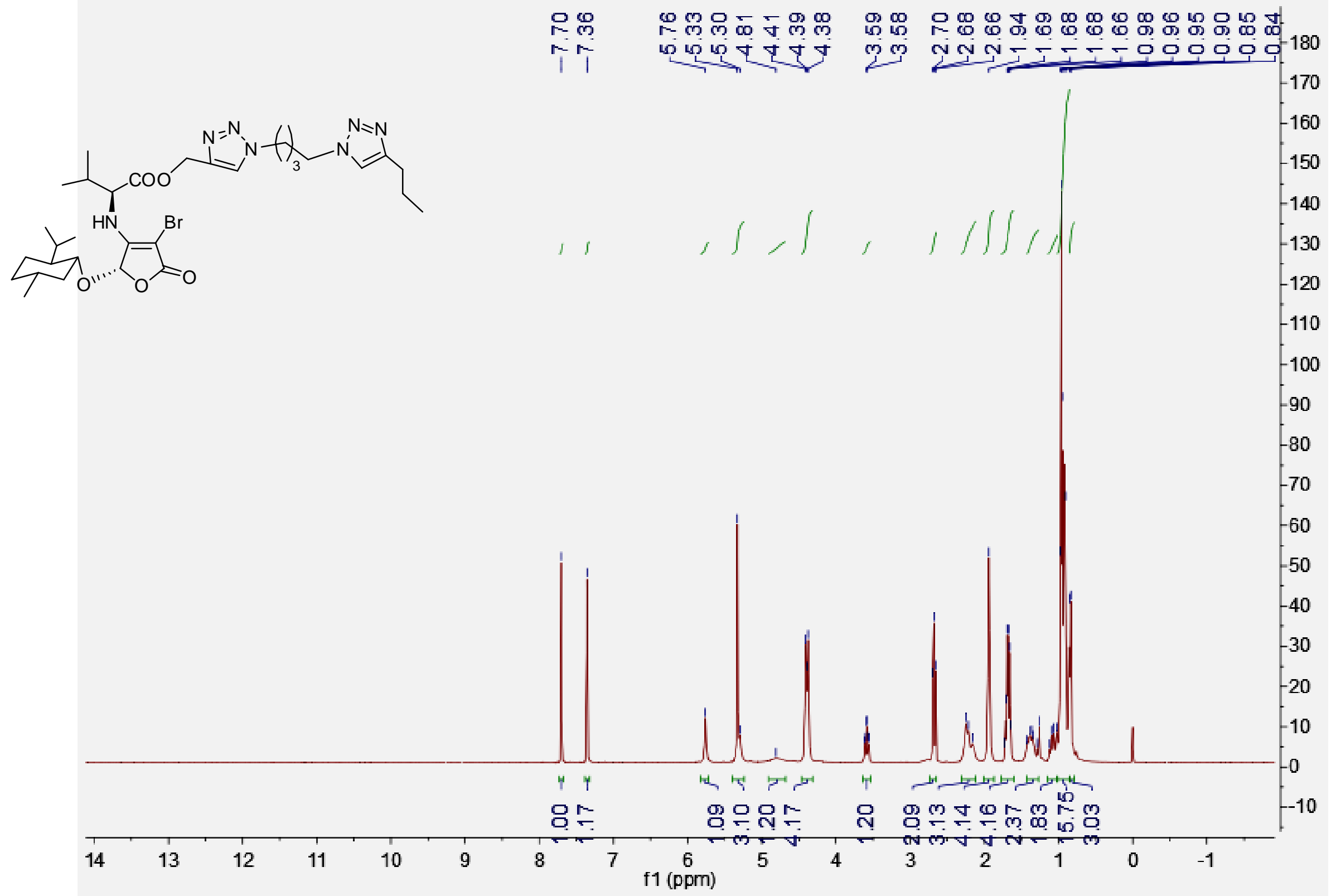




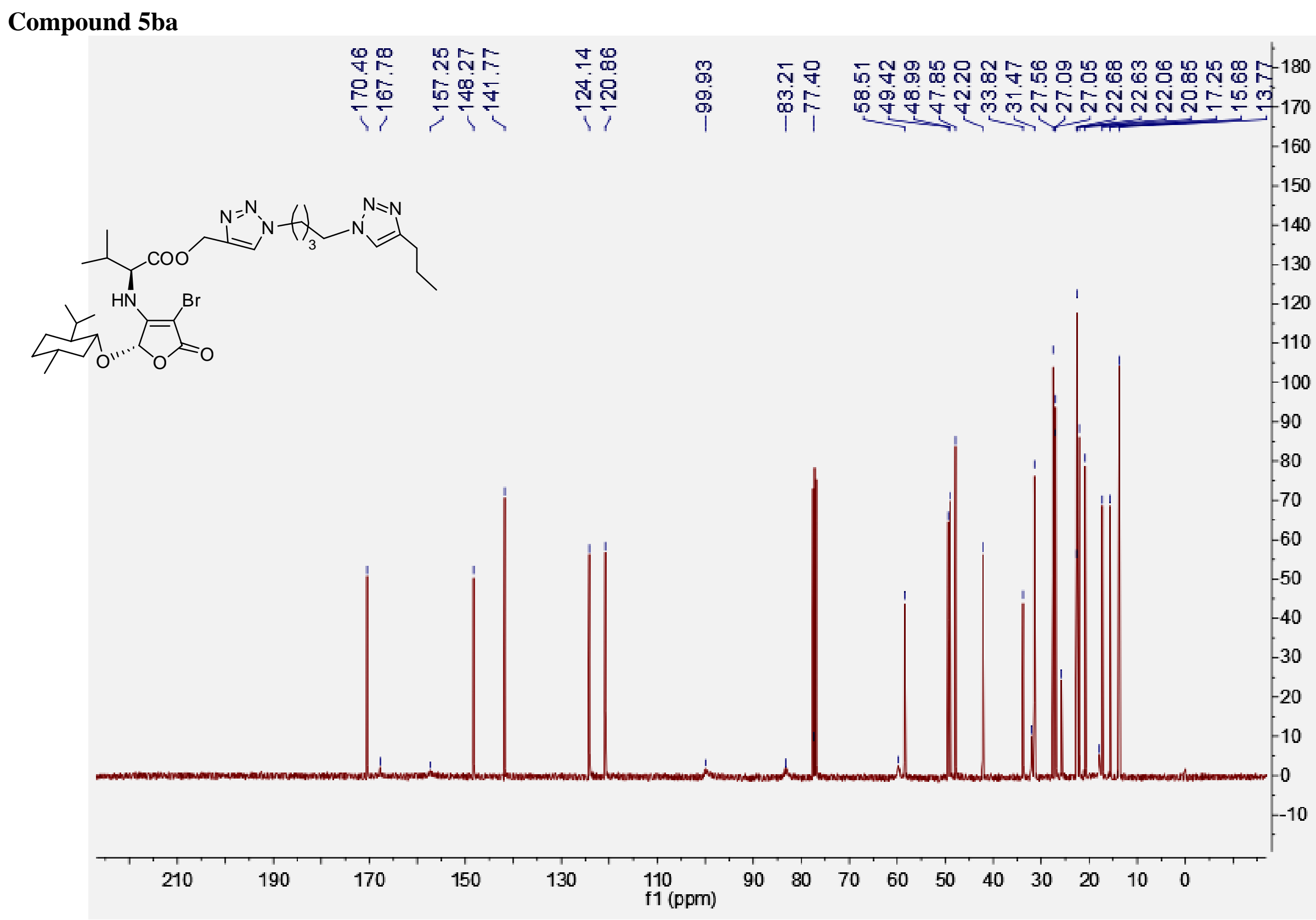




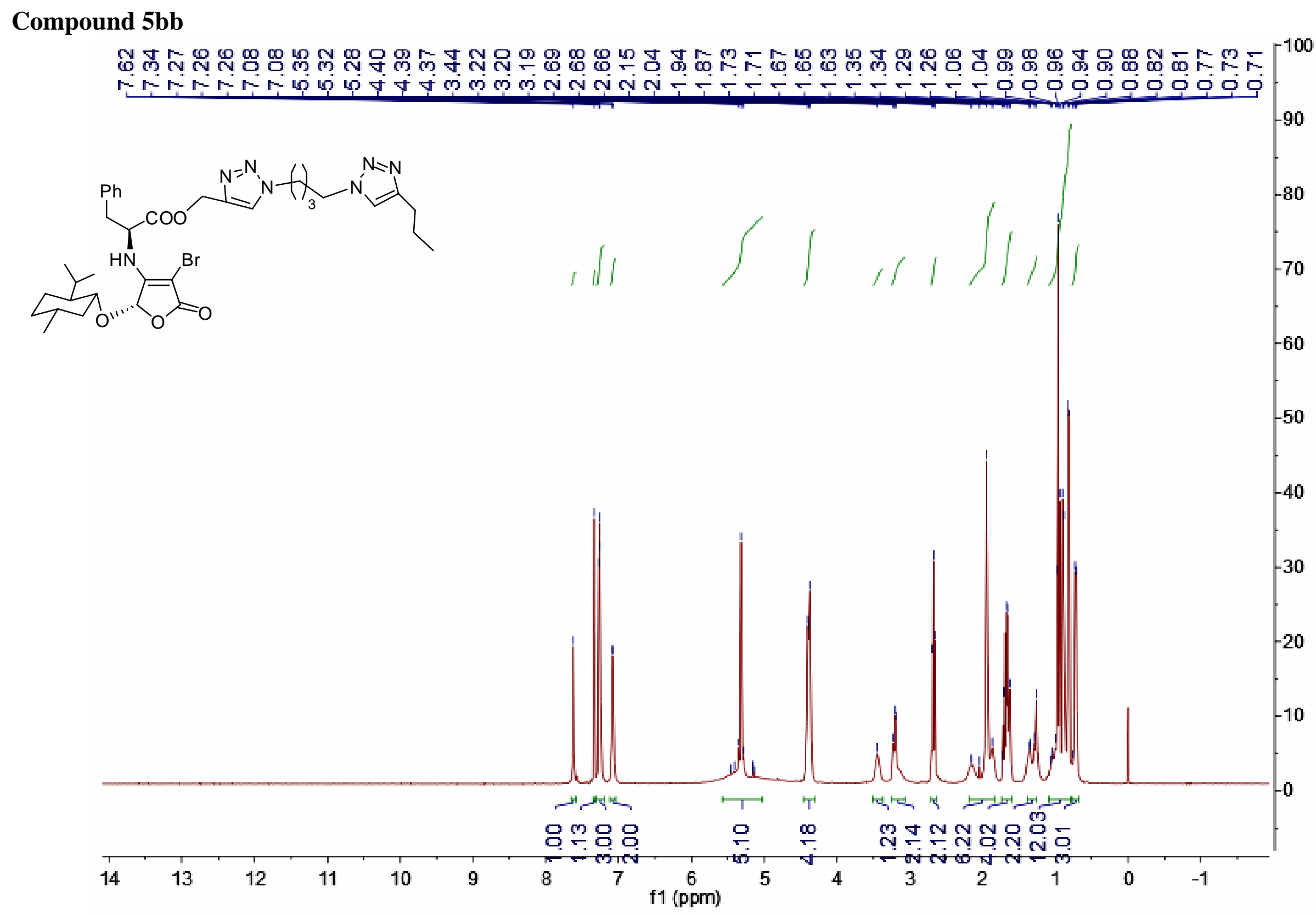




\section{Compound 5bb}

ต

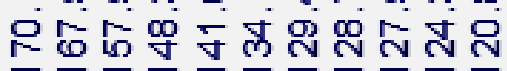

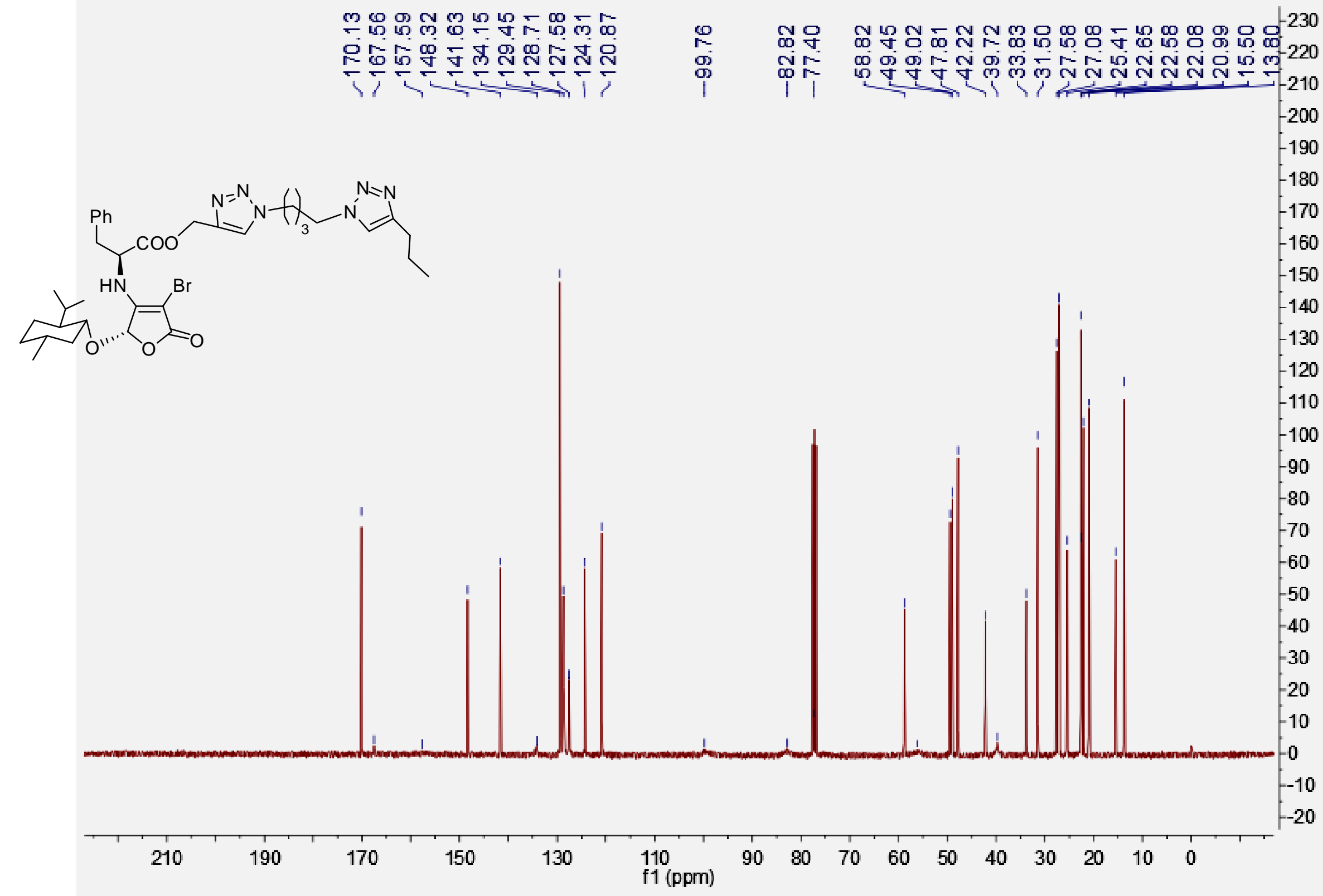




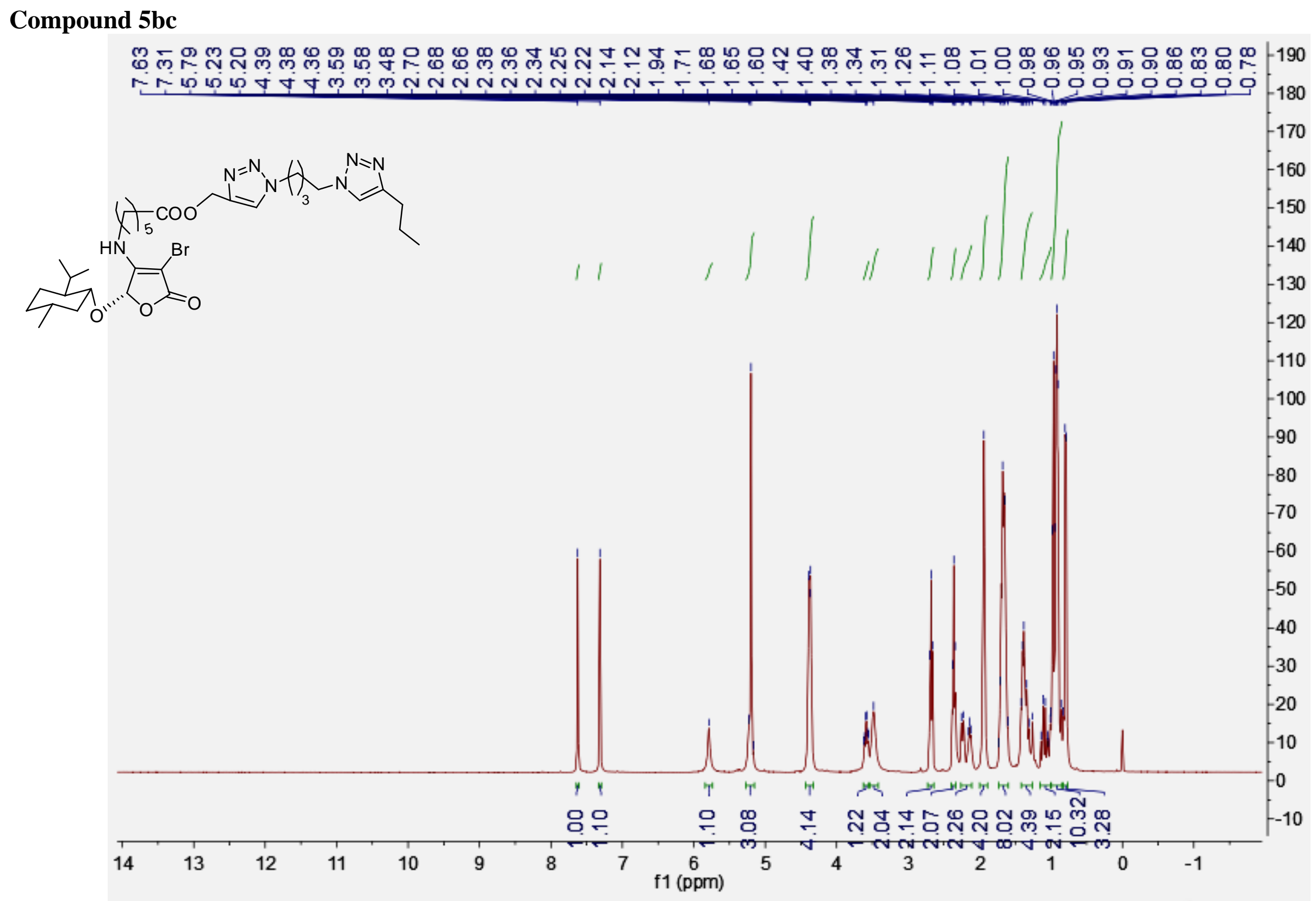




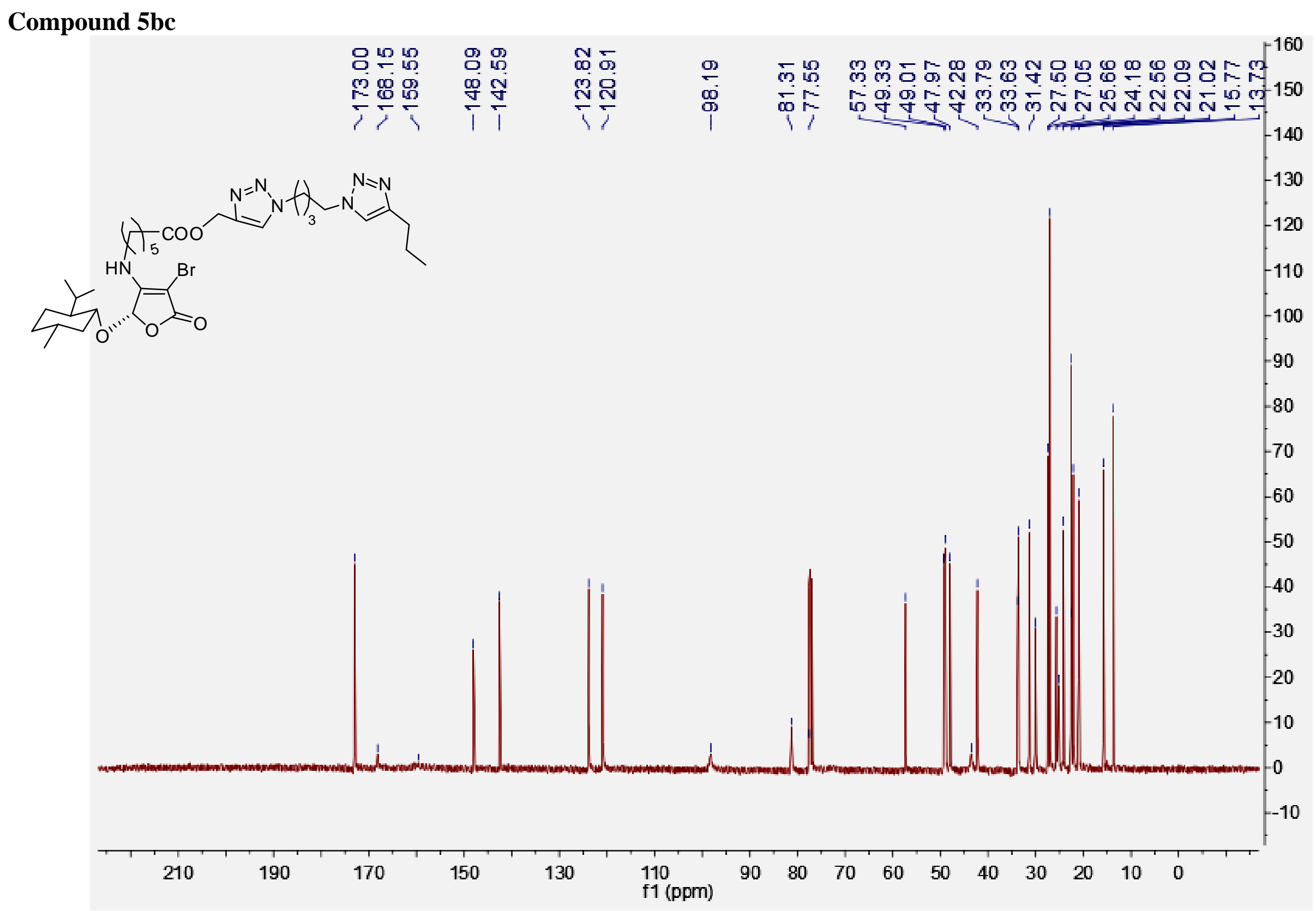




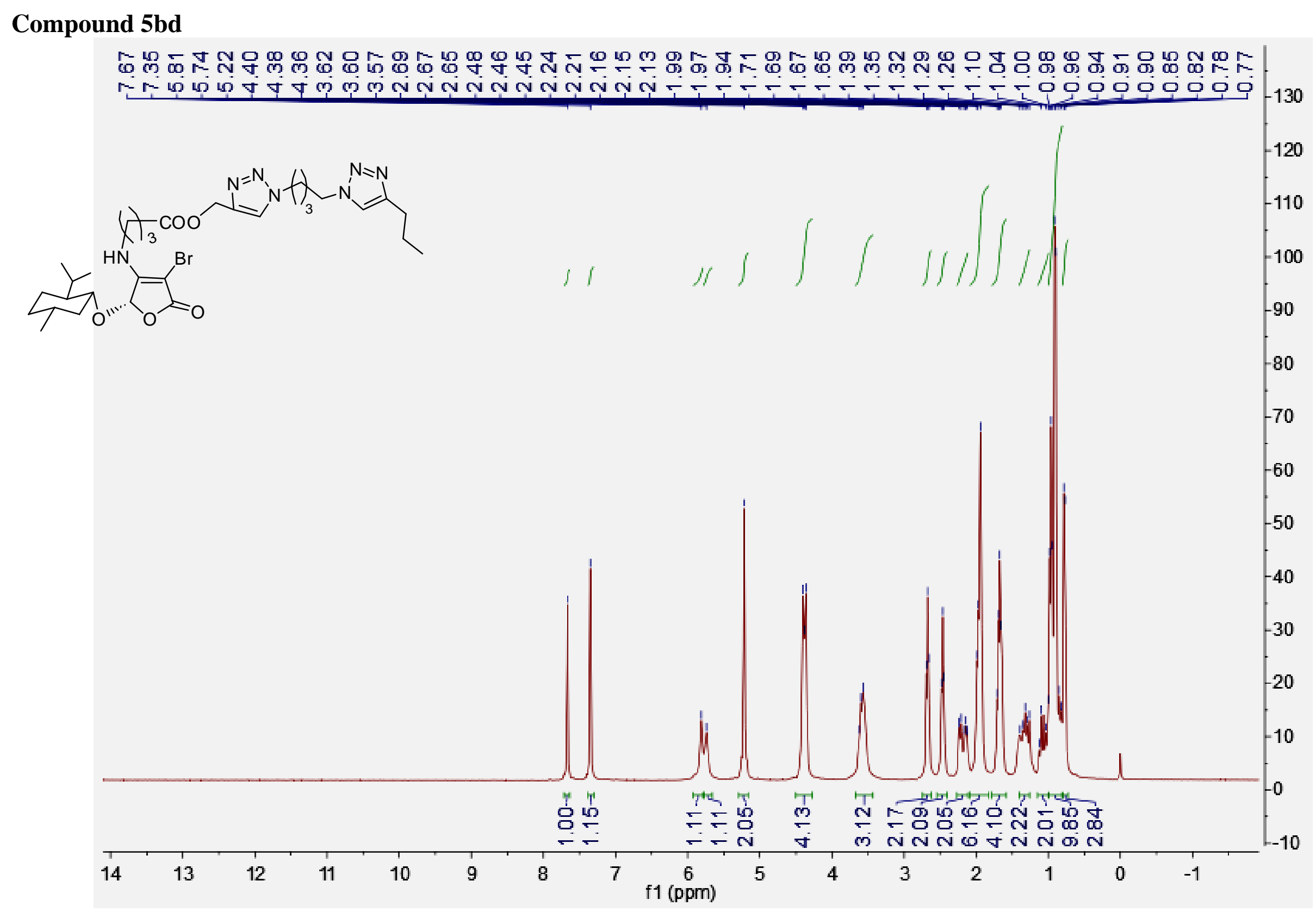




\section{Compound 5bd}

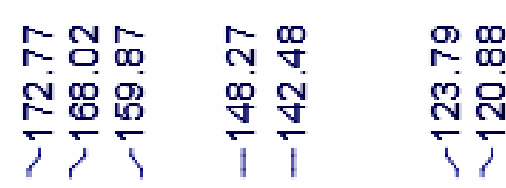

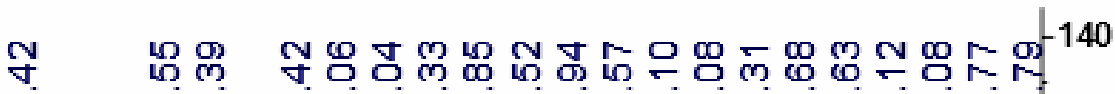

o
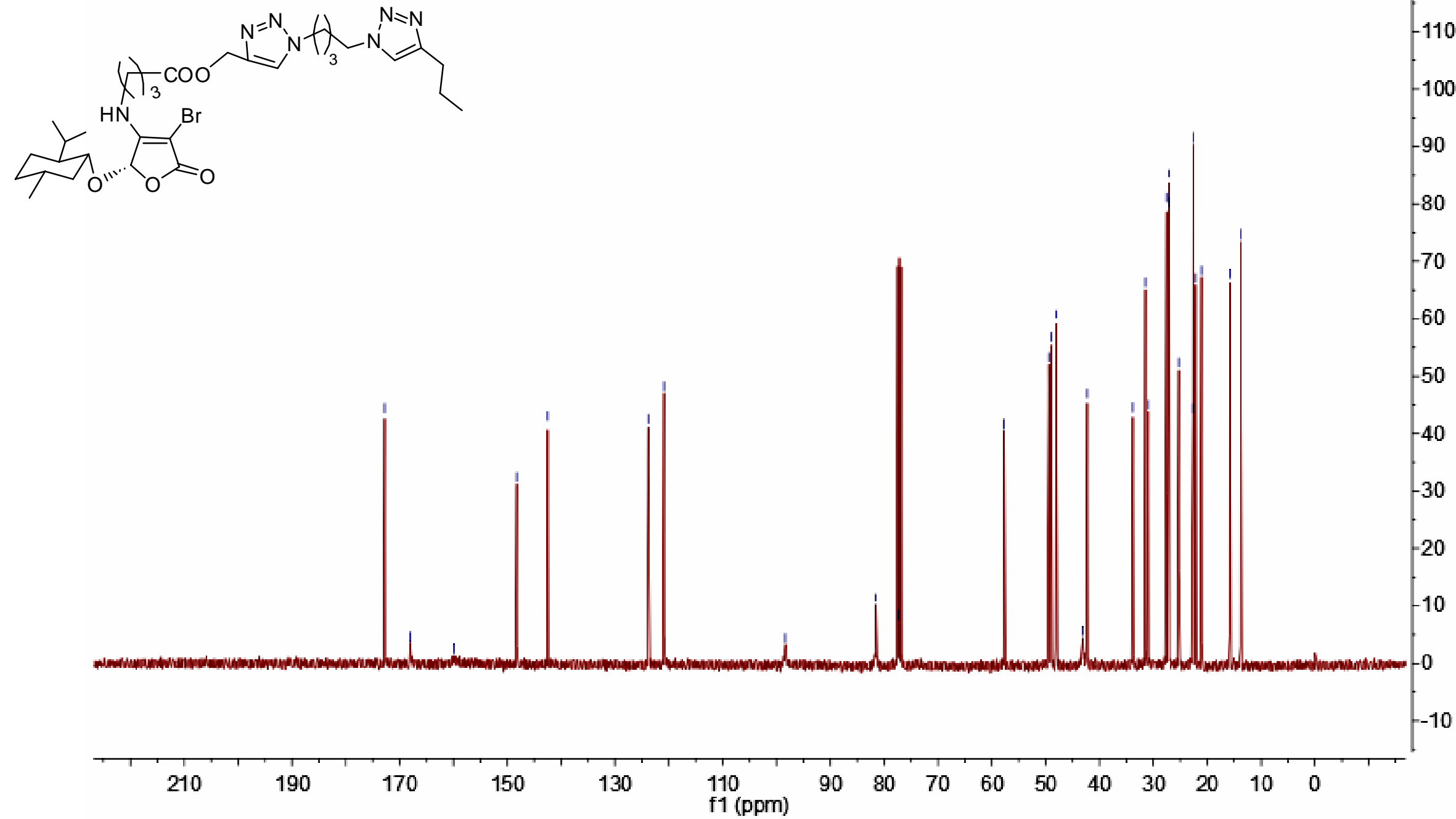


\section{Compound 5be}

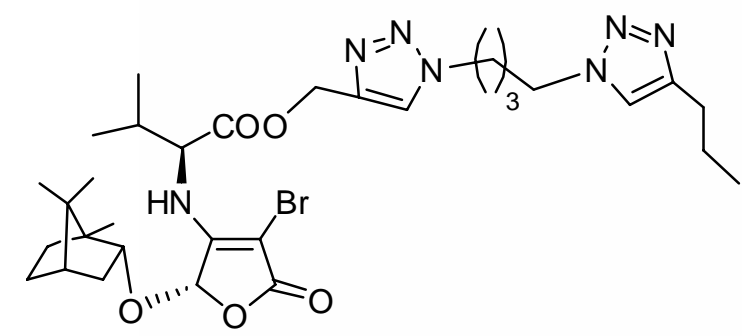

ம

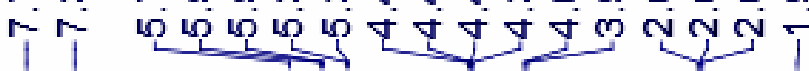

$-65$

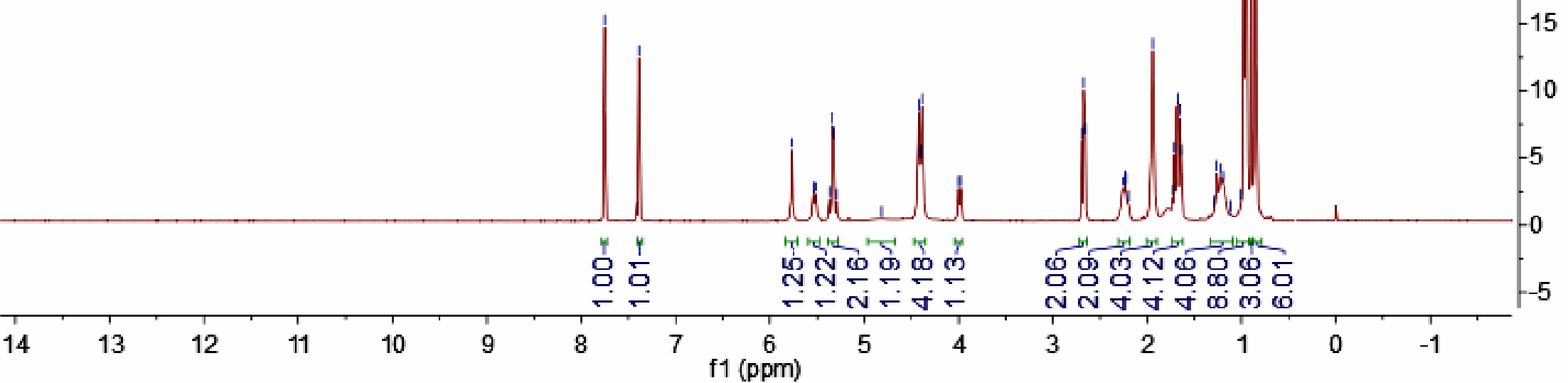




\section{Compound $5 b e$}

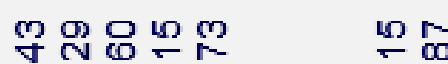

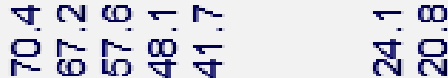

$\sum_{\infty}^{2} \frac{i}{i}$

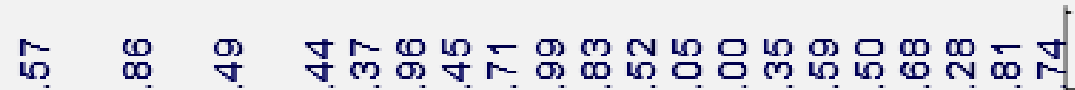

क

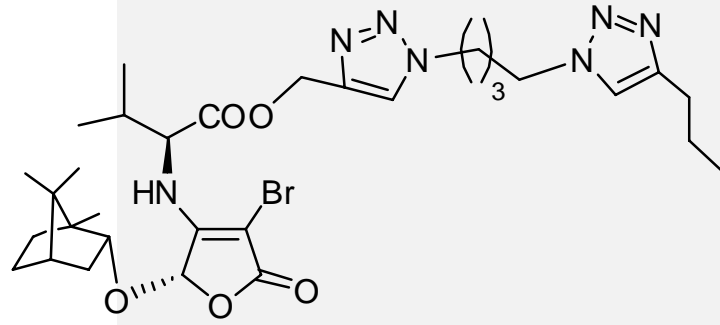




\section{Compound 5bf}

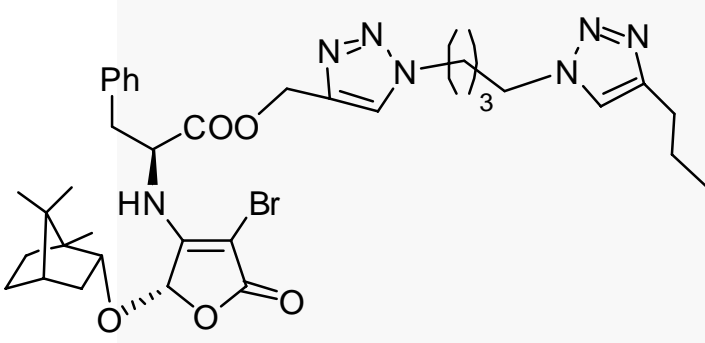

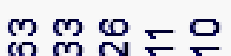

rim

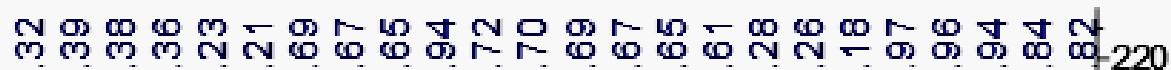

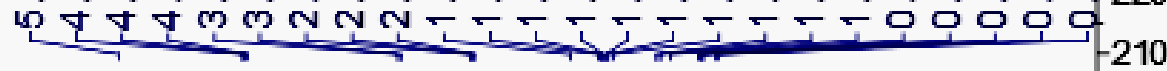

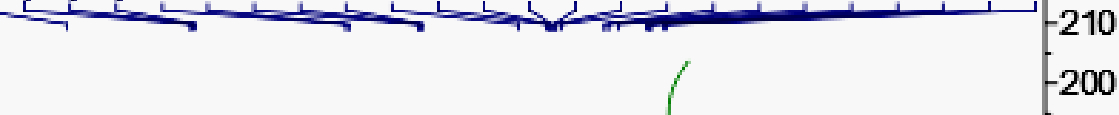

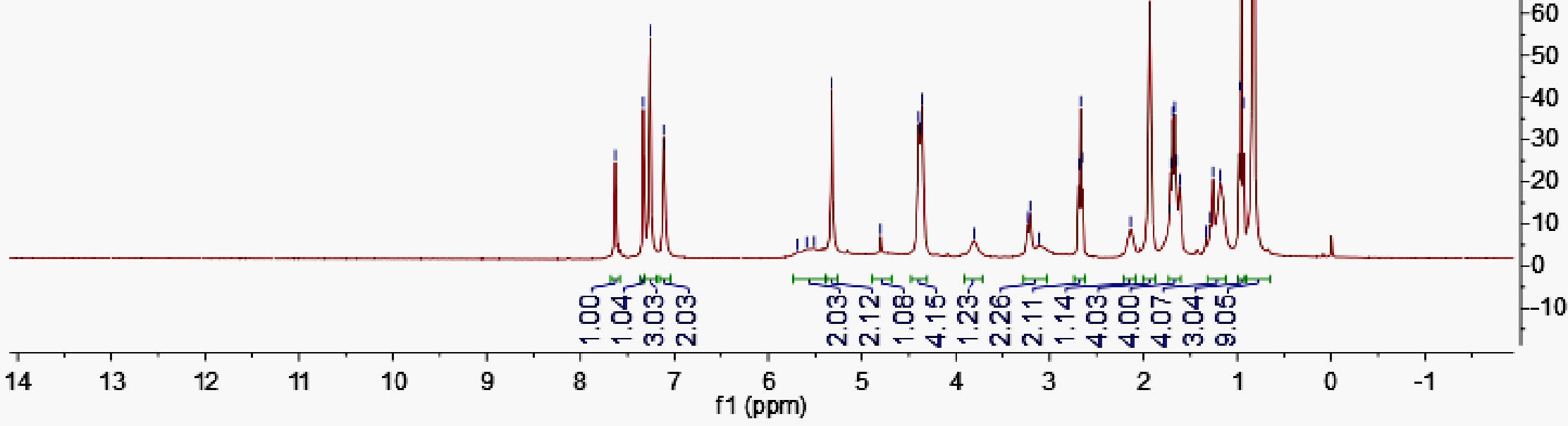




\section{Compound 5bf}

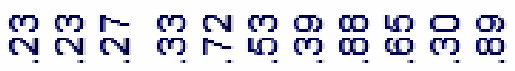

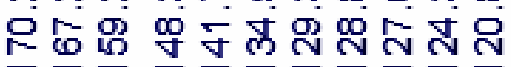

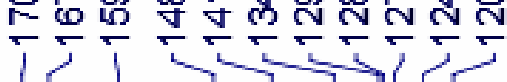

ल \ व

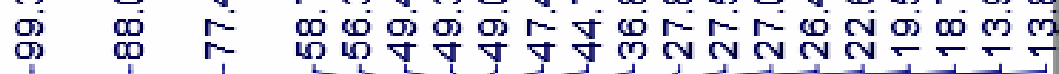

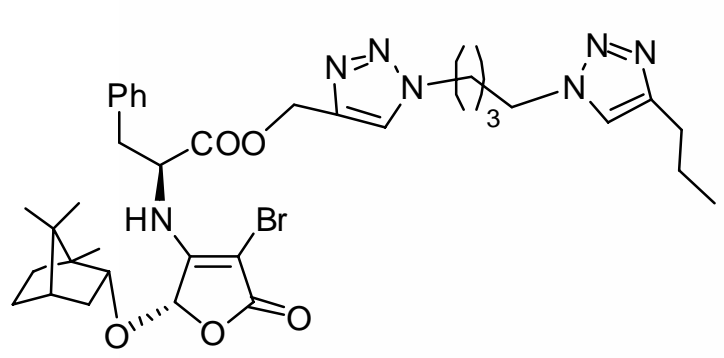

200

180

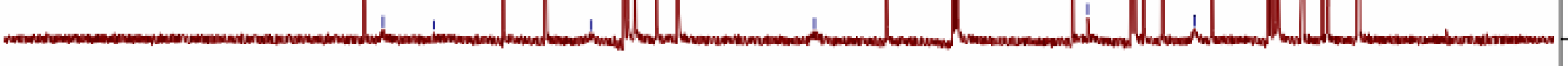




\section{Compound 5bg}

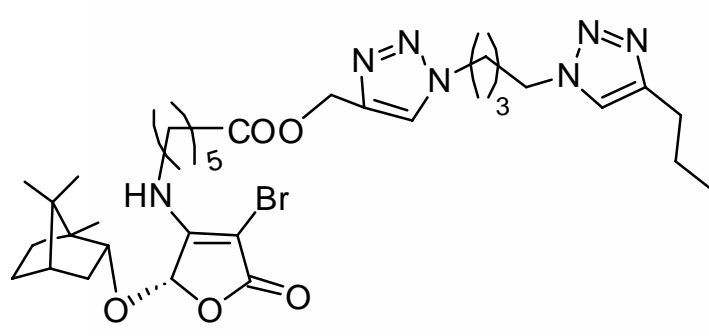

ֻ $\mathrm{m}$

mi

新只

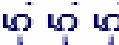

ले क्ल ले

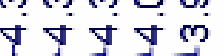

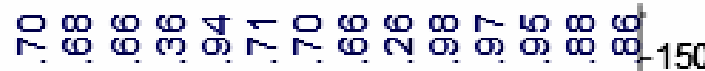
N N

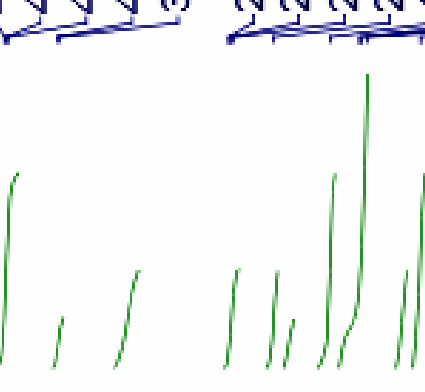

140

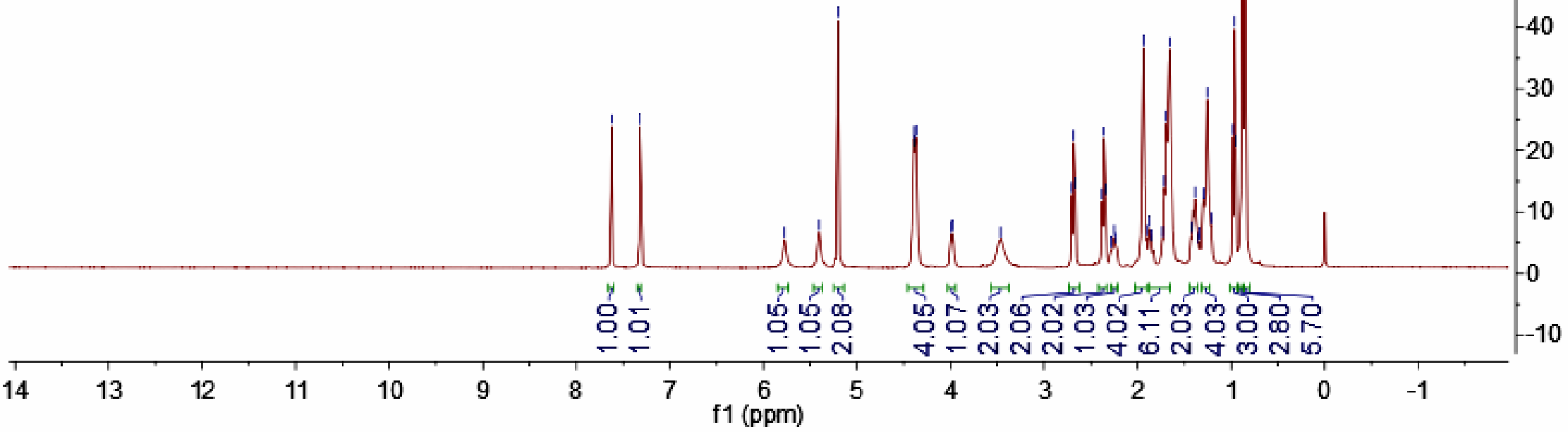




\section{Compound 5bg}

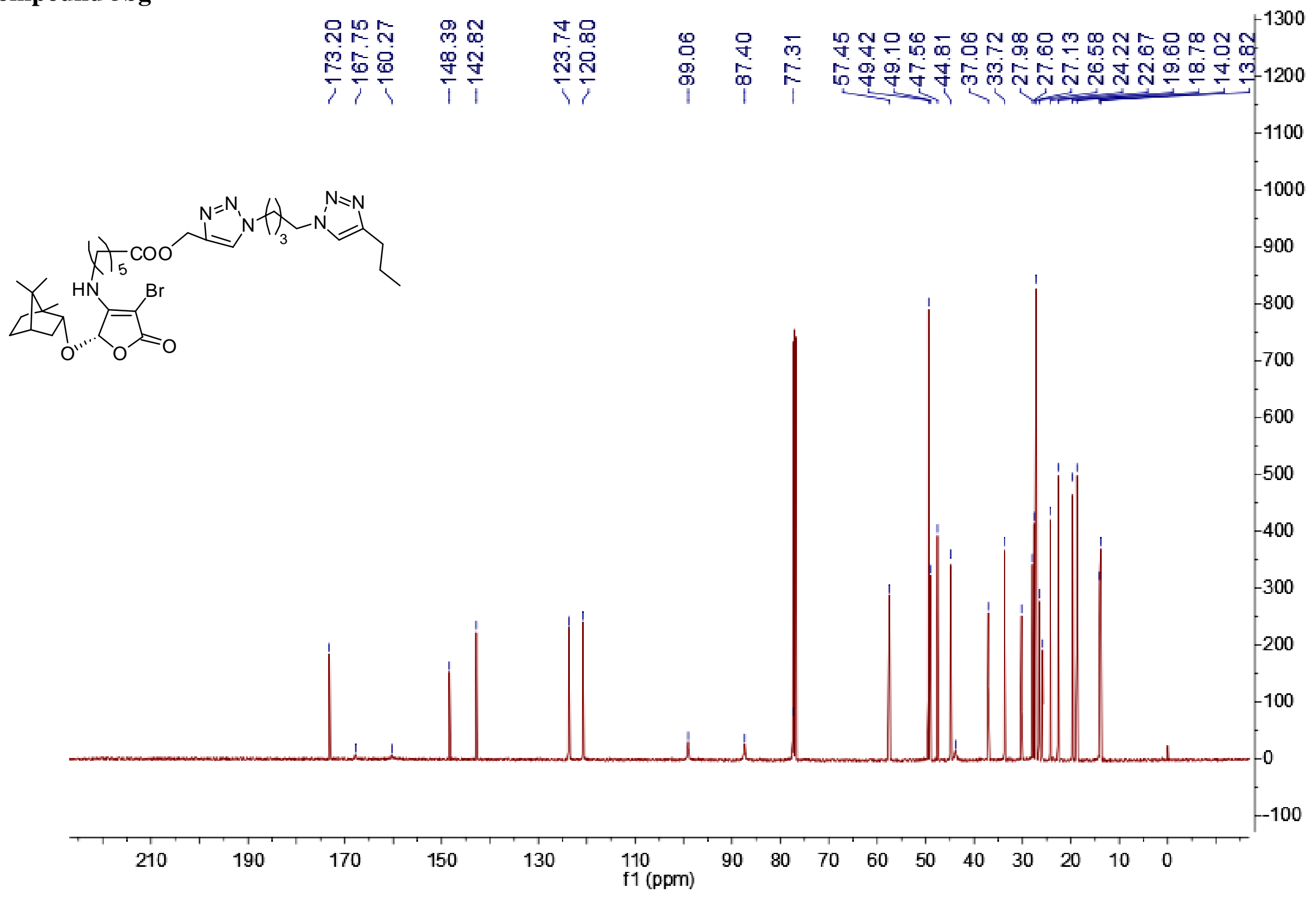




\section{Compound 5bh}

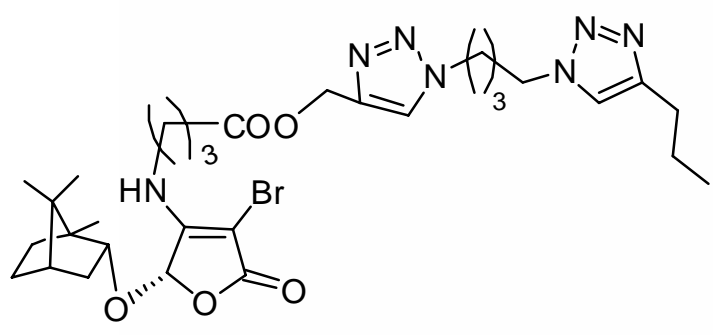

$\stackrel{\infty}{\infty}$

$\oplus \infty \bar{\infty}$

$-m$ m

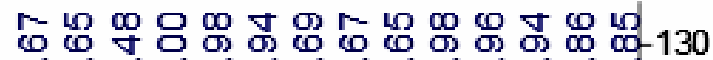

mi

फा एक्षणपल

inn

120

$-110$

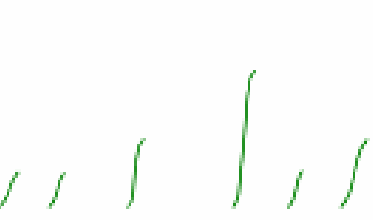

100

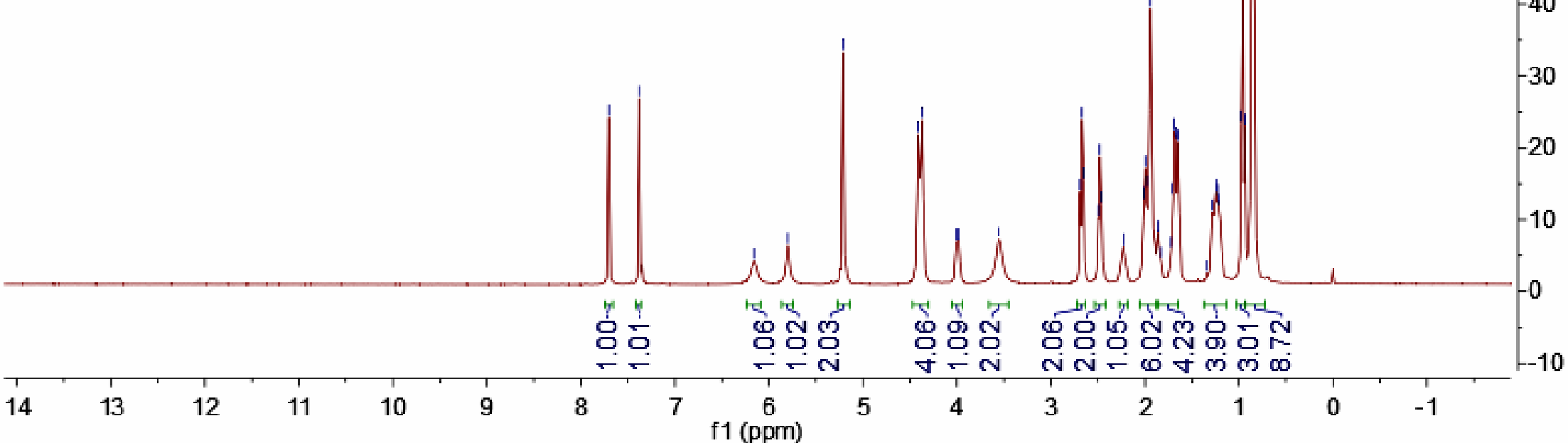




\section{Compound 5bh}

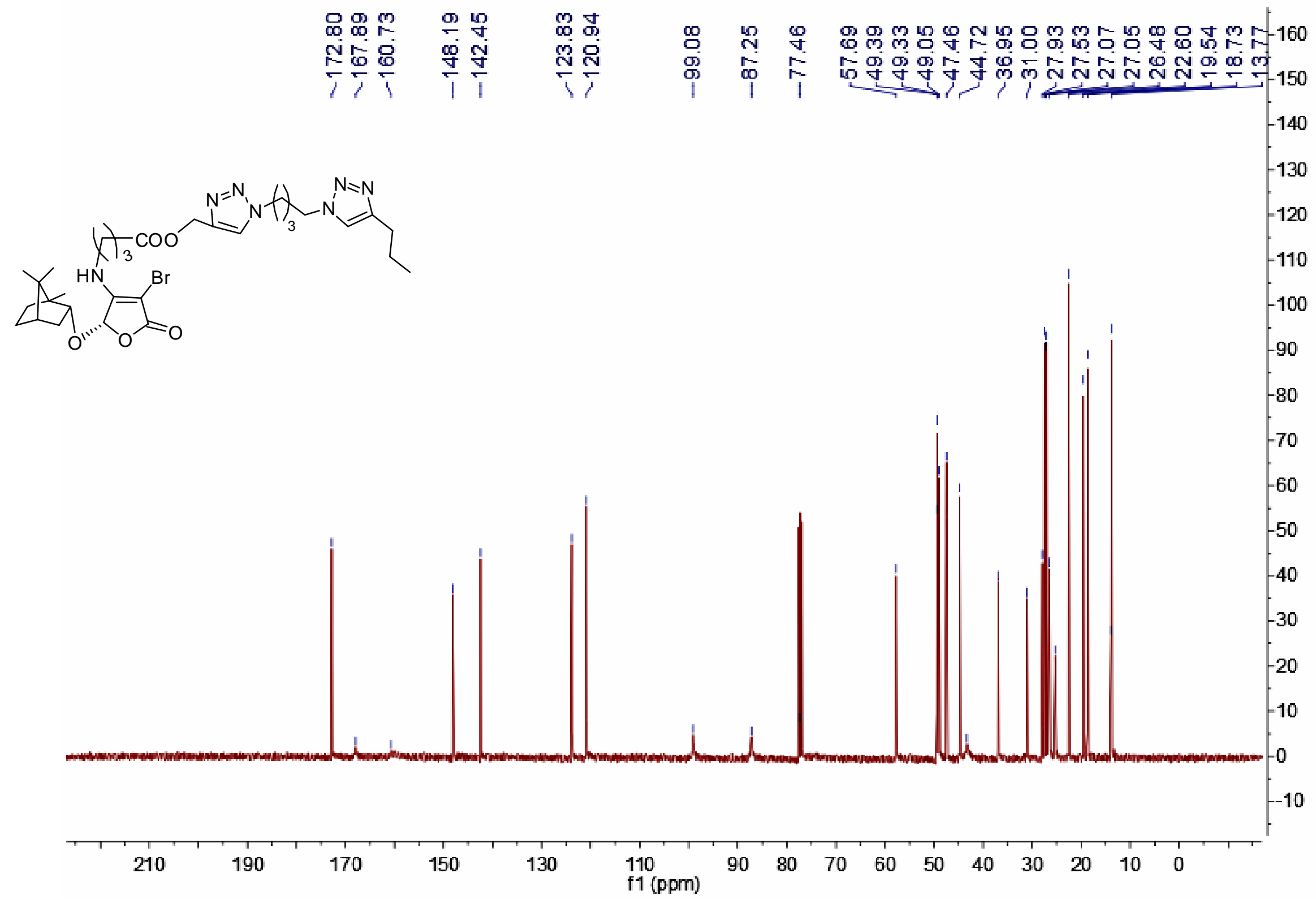

